International Journal of Bifurcation and Chaos, Vol. 17, No. 10 (2007) 3325-3371

(C) World Scientific Publishing Company

\title{
NONLINEAR METHODS OF CARDIOVASCULAR PHYSICS AND THEIR CLINICAL APPLICABILITY
}

\author{
NIELS WESSEL \\ University of Potsdam, Department of Physics, \\ Postfach 601553, 14415 Potsdam, Germany \\ wessel@agnld.uni-potsdam.de \\ HAGEN MALBERG \\ University of Karlsruhe, \\ Institute for Applied Computer Science/Automation, \\ Kaiserstr. 12, 76128 Karlsruhe, Germany \\ malberg@iai.fzk.de \\ ROBERT BAUERNSCHMITT \\ German Heart Center Munich, Clinic for Cardiovascular Surgery, \\ Lazarettstr. 36, 80636 Munich, Germany \\ Bauernschmitt@dhm.mhn.de \\ JÜRGEN KURTHS \\ University of Potsdam, Department of Physics, \\ Postfach 601553, 14415 Potsdam, Germany \\ jkurths@agnld.uni-potsdam.de
}

Received August 18, 2006

In this tutorial we present recently developed nonlinear methods of cardiovascular physics and show their potentials to clinically relevant problems in cardiology. The first part describes methods of cardiovascular physics, especially data analysis and modeling of noninvasively measured biosignals, with the aim to improve clinical diagnostics and to improve the understanding of cardiovascular regulation. Applications of nonlinear data analysis and modeling tools are various and outlined in the second part of this tutorial: monitoring-, diagnosis-, course and mortality prognoses as well as early detection of heart diseases. We show, that these data analyses and modeling methods lead to significant improvements in different medical fields.

Keywords: Cardiovascular physics; nonlinear data analysis and modeling; heart rate and blood pressure variability; baroreflex sensitivity.

\section{List of Abbreviations}

ACE alternating conditional expectation (algorithm)

ACM all cause mortality

AF atrial fibrillation

ApEn approximate entropy

BBI beat-to-beat interval 


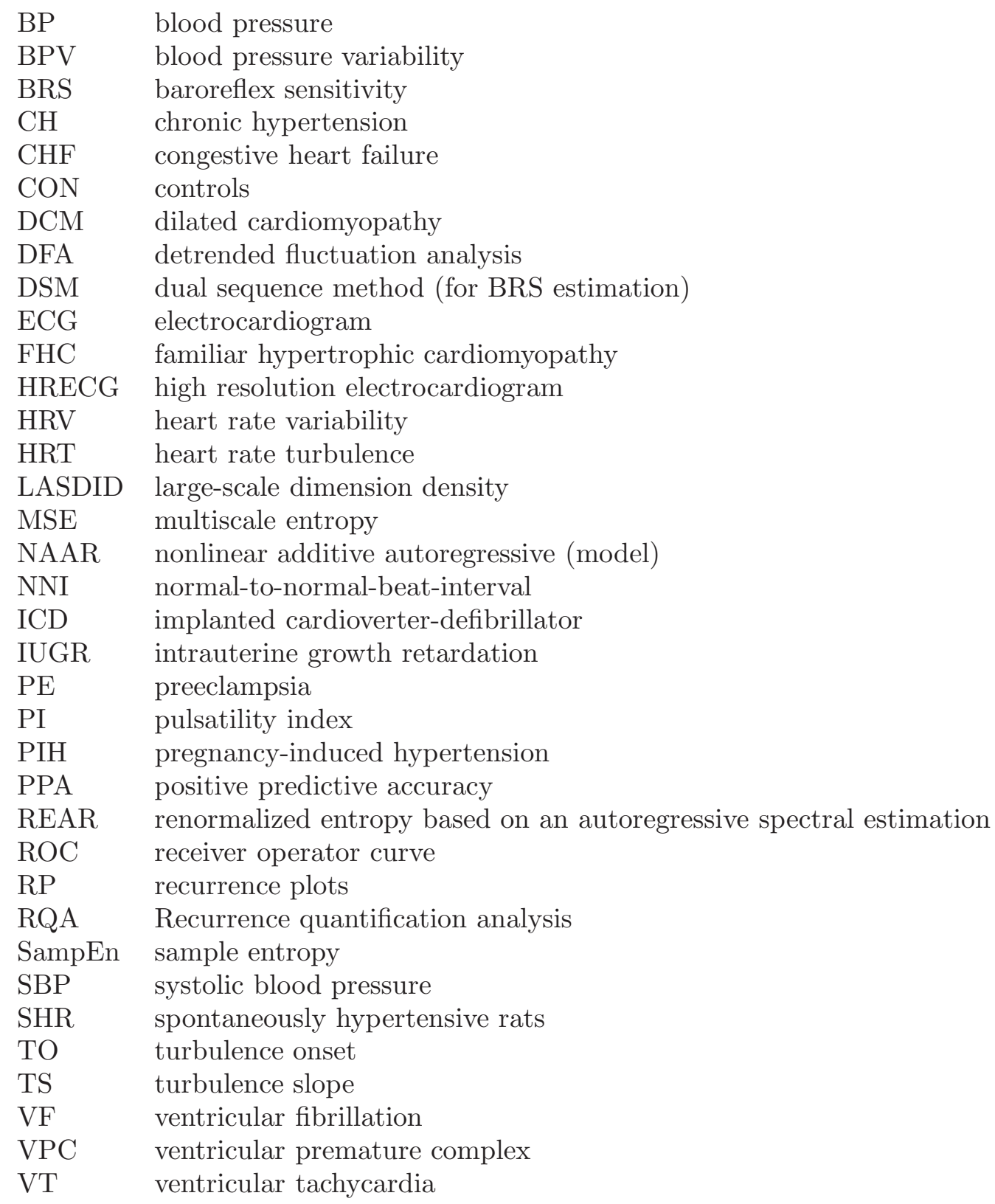

\section{Introduction}

Often data show complex structures which cannot be interpreted immediately, reaching from slowly moving periodic oscillations with stochastic influences to chaotic behavior with abrupt qualitative changes. Such a variety of complex dynamics can be found for example in cardiological data of patients with dynamic diseases. For cardiological data, the complexity of healthy dynamics can be understood to be an essential part of their capability to adapt to a varying environment. On the other hand, cardiac disease causes a reduced dynamic complexity and thus a reduced ability to adapt to environmental changes which is connected with a higher risk of life-threatening events.

Such difficult dynamics is mostly found in complex systems and cannot be explained by standard data analysis. Hence, the central demand of nonlinear dynamics aims exactly at such phenomena. In this tutorial, new nonlinear data analysis and modeling methods of cardiovascular physics are introduced and applied to clinical data. The first part of the work is focused on the methods developed in the field of cardiovascular physics, especially nonlinear data analysis and modeling of noninvasively measured biosignals with the aim to improve 
clinical diagnostics and to better understand cardiovascular regulation. The second part describes applications to clinically relevant problems.

Most cardiovascular diseases are characterized by an initial slow progression which subsequently may lead to abrupt qualitative changes [Zipes \& Wellens, 1998; Lakatta \& Levy, 2003]. This pathogenesis is a dynamic process and the associated cardiovascular parameters, such as heart rate variability (HRV), exhibit complex dynamics. It is well known that a metronomic heart rate is pathological; the healthy heart is influenced by multiple neural and hormonal factors that result in variations in RR-intervals. The disadvantage of linear parameters in analyzing such processes is the limited information about the underlying complex system. They often require stationarity and do not take into account nonlinear correlations. On the other hand, a classical nonlinear description, involving correlation dimension or Lyapunov exponents estimation [Almog et al., 1996; Babloyantz \& Destexhe, 1988; Glass \& Mackey, 1988; Goldberger et al., 1984; Kobayashi \& Musha, 1982; Lombardi et al., 1996b], suffers from the curse of dimensionality. Mostly, there are not enough points in the (often nonstationary) time series to reliably estimate these nonlinear measures.

Therefore, we favor measures of complexity another approach within nonlinear dynamics which are able to quantitatively characterize the dynamics even for rather short time series [Schwarz et al., 1993; Kurths et al., 1995; Voss et al., 1996b; Voss et al., 1998; Wessel et al., 2000c].

Several techniques to analyze heart rate and blood pressure variability (BPV) data have been proposed in the literature [Task Force Heart Rate Variability, 1996]. However, until today no technique exists that reliably describes these types of cardiovascular regulation. A main focus of this tutorial is to discuss different approaches to cardiological signal analysis to get a better understanding of the underlying processes. In our opinion, the only way to develop appropriate models for cardiovascular regulation is to study the systems via sophisticated methods and apply them to data of different pathologies.

Annually, in the United States approximately 450,000 people die due to sudden cardiac death [Alberte \& Zipes, 2003; Barron \& Lesh, 1996; Damiano, 1992]. The accurate and reliable identification of patients who are at high risk of sudden cardiac death is an important and challenging problem.
HRV and BPV parameters, calculated from time series of beat-to-beat intervals (BBI), have been used to predict the mortality risk in patients with structural heart diseases [Kleiger et al., 1987; Tsuji et al., 1996]. Moreover, they were used for estimating medicamental and therapeutical effects as well as for physiomonitoring [Karemaker \& Lie, 2000; Mortara et al., 1997]. Further, cardiovascular interactions are analyzed to gain information for the development of cardiac pacemakers or implantable cardioverter-defibrillators [Sugiura et al., 1991; Klingenheben \& Hohnloser, 2003]. The continuous and ongoing development in variability analyses all have the same aim:

- Gaining new physiological information for diagnostics, prognosis and risk stratification, for clinical treatment, pharmacologic research and genetic research,

- Minimization of the patients risk due to the improved analysis of especially noninvasive biological signals,

- Substitution of invasive diagnostic procedures by improved and substantial cost saving diagnostics.

A fundamental step to analyze cardiovascular variability was the invention of the electrocardiogram (ECG) for noninvasive detection of cardiac excitation propagation by EINTHOVEN in 1902 [Einthoven, 1902]. The noninvasive continuous blood pressure (BP) measurement was developed several decades later. The fundamental work was patented in 1969 by PENAZ and published afterwards [Penaz, 1973]. Technical realizations for noninvasive continuous BP measurement were available starting from the 80's with the "Finapres" device (company Ohmeda, USA). Surprisingly, the basic scientific knowledge of cardiovascular variability analyses had been known a long time before, and is represented in the following list [Rompelman \& Ten Voorde, 1995; Man in't Veld et al., 1995]

- S. HALES (1733): Detection of respiratory induced BP-fluctuations

- C. LUDWIG (1846): Detection of respiratory induced heart rate (HR) fluctuations

- L. TRAUBE (1865): Influence of curare to BP in an animal model

- E. DECYON, L. LUDWIG (1866): Neurogenetic feedback between heart and circulation

- E. HERING (1868): Respiratory influence to BP-waves 
- S. MAYER (1876): Detection of Mayer-waves; lower frequency than respiratory induced BPfluctuations

- G. KÖSTER, A. TSCHERNAK (1903): Detection of vagal nerves in the aorta

- A. HEPPINGER, C. HESS (1948): Detection of sympathovagal balance.

The investigation of the mutual influence of $\mathrm{HR}$ and $\mathrm{BP}$ in the control of autonomous regulation nowadays is a main research topic [La Rovere et al., 2003]. A comprehensive methodological characterization of the beat-to-beat cardiovascular regulation, however, is missing until today. Moreover, there exists no "golden parameter" whose measurement is able to reliably describe and estimate all risks. From time to time there is an excessive enthusiasm of some scientists claiming that their new developed parameter is better than previously used ones. However, none of the known risk predictors probably is or can ever serve as an omnipotent outcome determinant. Nevertheless, characteristic phenomena of cardiovascular regulation are very exactly detectable with specifically adapted methods [Wessel et al., 2003].

The large complexity of cardiovascular regulation, with its multiplicity of hormonal, genetic and external interactions, requires a multivariate approach based on a combination of different linear and nonlinear parameters [La Rovere et al., 2001; Bauernschmitt et al., 2004; Wessel et al., 2004c]. We have recently demonstrated that a multivariate approach with HRV parameters including nonlinear methods as well as the combination of HRV measures with clinical parameters like the ejection fraction, the complexity of ventricular arrhythmias or the signal-averaged electrocardiogram, significantly improves the results of risk stratification [Voss et al., 1998]. Moreover, in numerous clinical studies [Barron \& Lesh, 1996; Lombardi et al., 1996b; Lombardi et al., 1996a; Voss et al., 1996b; La Rovere \& Schwartz, 1997; La Rovere et al., 2001] it was demonstrated, that a multiparametric analysis of the cardiovascular regulation increases the diagnostic information. However, one has to consider some characteristics when using mathematical approaches for the analysis for physiological systems (MILHORN [Milhorn, 1966]). We single out the following valuable characteristics:

- Relations between variable biological systems are usually nonlinear,
- Biological control systems have multiple feedback loops and the dynamics result from the interplay between them,

- Biological systems are much more complicated than physical systems.

Considering these rather system-theoretical characteristics, the development of nonlinear and also knowledge-based methods should lead to a diagnostic improvement in risk stratification. Accordingly, first applications of nonlinear dynamics tools in medical diagnostics have been met with success over the last decade [Focus Issue: Dynamical Disease: Mathematical Analysis of Human Illness, 1995; Kantz et al., 1998]. So far, however, research was limited either to only analyze data [Goldberger et al., 1988; Yamamoto \& Hughson, 1994a; Cerutti et al., 1996; Poon \& Merrill, 1997; Ivanov et al., 1999; Schmidt et al., 1999a; Yang et al., 2003; Wessel et al., 2004c], or develop models [Garfinkel et al., 1992; Wikswo et al., 1995; Gray et al., 1998; Witkowski et al., 1998; Ditto \& Showalter, 1998; Focus Issue: Mapping and Control of Complex Cardiac Arrhythmias, 2002; Special Issue: Virtual Tissue Engineering of the Heart, 2003]. A further aim of this tutorial, therefore, is, to go a qualitatively new step: the combination of data analysis and modeling. This model-based nonlinear-dynamic data analysis of noninvasively measured biosignals leads to an improved understanding of the cardiovascular regulation. Applications of these research results are manifold: Monitoring-, diagnosis-, course- and mortality prognoses as well as the early detection of heart diseases. With respect to clinical applications, there is an extraordinary demand for new computer-controlled diagnostic methods to obtain a more exact and differentiated picture of the possibly damaged heart and to develop new biomedical devices for risk stratification.

The present work represents a summary of new and innovative methods developed by our group, which offer a lot of clinical applications and may sometime replace or complement some invasive and expensive diagnostics.

\section{Methods of Cardiological Biosignal Processing}

\subsection{State of the art in clinical practice}

Clinical diagnostics in cardiology includes a lot of invasive as well as non-invasive procedures. Only 
few of them, such as electrocardiography, offers the possibility to characterize the dynamics of the cardiovascular system over time (from minutes to hours and days). Using portable Holter devices, the study of beat-to-beat changes in the ECG is possible under physical load and over the whole day. Other diagnostic tools, as for example imaging, invasive or biochemical procedures, are only able to represent the heart functionally and morphologically for a rather short temporal interval.

The everyday routine in a hospital only allows specialized and expensive devices for variability analyses. This is all the more unsatisfactory because numerous clinical studies proved the diagnostic power of variability analyses. It is state of the art today, to detect single beats in the ECG and to analyze how the difference between two successive beats evolves. Already in 1996 guidelines to analyze this variability were published by the Task Force of the European Society of Cardiology and the North American Society of Pacing and Electrophysiology: "Heart rate variability: Standards of measurement, physiological interpretation and clinical use" [Task Force Heart Rate Variability, 1996]. However, they have not become generally accepted as standard diagnostic tools so far. There are two reasons for the low acceptance: firstly, the deficits in the cardiological education for this new diagnostic field and secondly, the reduced availability of commercial devices for variability analyses.

The analysis of the heart rhythm is only one substantial component of the interpretation of electrocardiograms. Several medical questions can already be answered by visual inspection of the ECG paper strips themselves, e.g. whether the electrical excitation in different locations of the heart (sinus node, atrium, AV node, ventricle) is normal or not. Moreover, arrhythmias of different origin can be detected (e.g. sinus bradycardia/tachycardia, atrial tachyarrhythmias, ventricular tachycardia/fibrillation, supraventricular beats) as well as the sick sinus node syndrome and other electrical pathologies. In addition to the arrhythmic considerations several morphologic disturbances, such as atrioventricular block, left/right bundle branch block, WPW syndrome, atrioventricular dissociation as well as injury currents (e.g. after myocardial infarction) can be visually identified [Zareba et al., 2001].

A substantial parameter in the ECG is the T-wave (Fig. 1), which had mostly been visually analyzed in the past. It is now possible to analyze

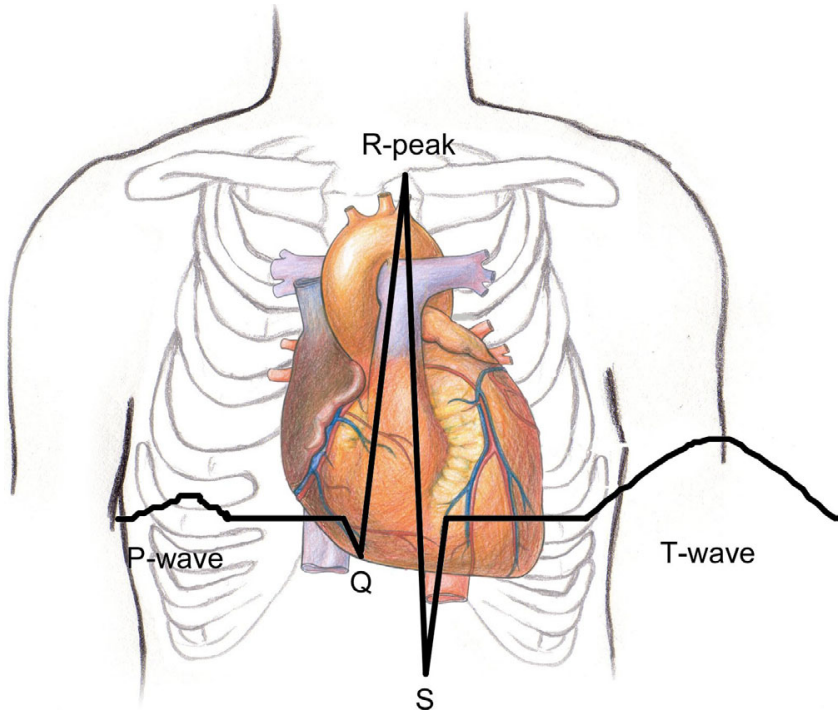

Fig. 1. Schematic representation of the PQRST-complex in the electrocardiogram.

it in a computer-assisted way. Already in 1909 HERING described [Hering, 1909] an electrical alternans of the T-wave which one year later was correlated with cardiac morphology by LEWIS [Lewis, 1910]. High amplitude T-wave alternans are rarely seen in patients at risk for sudden cardiac death. However more recent studies showed that low amplitude T-wave alternans discriminates patients with ventricular tachycardia (VT) or ventricular fibrillation (VF) from those without VT or VF [Rosenbaum et al., 1996]. The analysis of the ST-segment is a standardized method of ECG analysis, but surprisingly no variability of this segment has been considered so far [Lehtinen et al., 1996; Suurkula et al., 2001]. There are two types of ST-T wave abnormalities which are very common and may be seen in any lead of the ECG: an ST segment depression and an ST segment elevation. Nonspecific T-waves and STsegments changes may also be seen in healthy individuals and can be caused by anemia, fever, drugs or acute infections. Specific ST-segments changes may be seen in association with various diseases, such as ischemia, myocardial injury, pericarditis or left ventricular hypertrophy [Kudenchuk et al., 1998]. A further standardized clinical diagnostic tool is the analysis of the late potentials [Breithardt et al., 1991] in the high resolution ECG, which however is not established in practice yet. Cardiac late potentials are low amplitude electrical signals (less than $1 \mathrm{mV}$ ) that occur after the normal QRS and represent delayed conduction in damaged or diseased ventricular myocardium. They cannot be 
seen in normal ECGs, but are revealed only after signal averaging because of background noise. The occurrence of late potentials themselves can be a harbinger of serious ventricular tachyarrhythmias, but the positive predictive value is low [Steinbigler et al., 1998].

\subsection{Linear analysis of heart rate and blood pressure variability}

Spontaneous fluctuations of cardiovascular signals such as heart rate and blood pressure had already described more than hundred years ago [Ludwig, 1847; Koepchen, 1959; Wolf et al., 1978]. However, the physiological interpretation of these variabilities is still an interesting and exciting research area. The fluctuations of heart rate and blood pressure represent not only oscillations around a fixed value. They are the expression of several influences, such as respiration and different self-regulating rhythms.

The basis of nearly all HRV studies is the ECG recording. The trigger point to detect the beatto-beat variations in the ECG should be the time difference between successive sinus node activities, visible as the $\mathrm{P}$-waves in the ECG. This wave, however, has a small amplitude and is sometimes difficult to detect (Fig. 1). Therefore as a compromise the distance between two successive R-peaks, which can be determined relatively simply, is used for estimating the HRV. Figure 2(a) shows the basic extraction principle of beat-to-beat $(\mathrm{RR})$ intervals from the ECG. To get more insight into cardiovascular regulation, simultaneous blood pressure signals are recorded additionally to the ECG. The lower part of Fig. 2 demonstrates how information about the beat-to-beat variation of systolic and diastolic pressure values is determined. Plotting the actual RR-intervals or the pressure values versus time, we get the tachogram/systogram/diastogram (Fig. 3).

Short-term heart rate regulation is accomplished mainly by neural sympathetic- and parasympathetic-mediated cardiac baroreflexes and peripheral vessel resistance, whereas long-term regulation is achieved by hormonal pathways as well as other systems like the renin-angiotensin-system [Berntson et al., 1997].

The analysis of HRV has become a powerful tool for the assessment of autonomic control. HRV measurements have proven to be independent predictors of sudden cardiac death after acute myocardial infarction, chronic heart failure or dilated cardiomyopathy [Kleiger et al., 1987; Tsuji

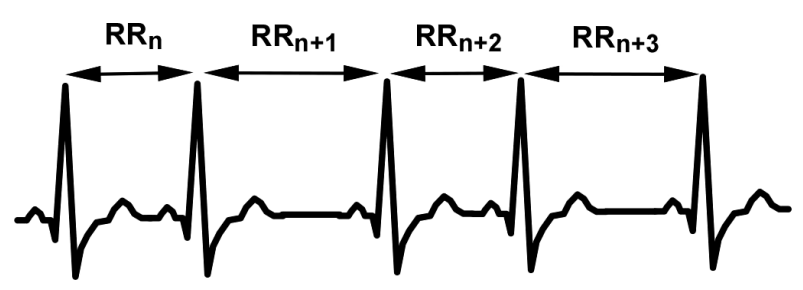

(a)

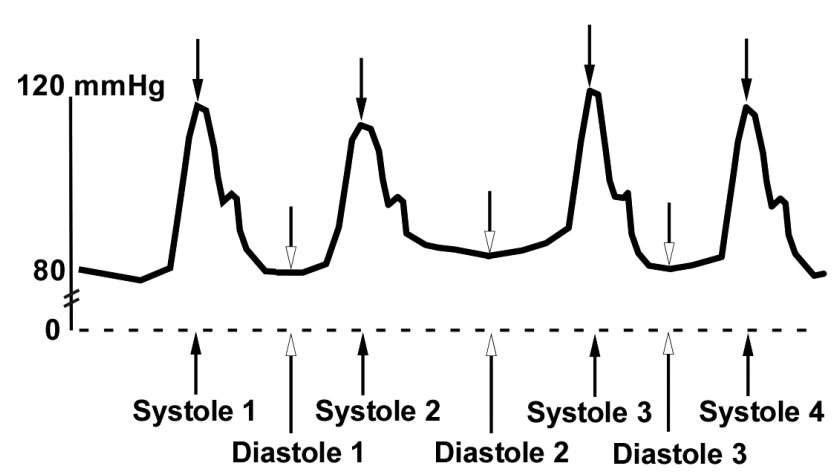

(b)

Fig. 2. Scheme of beat-to-beat interval extraction: The RRintervals from the ECG (a) as well as the systolic and diastolic blood pressure values from the blood pressure curve (b).

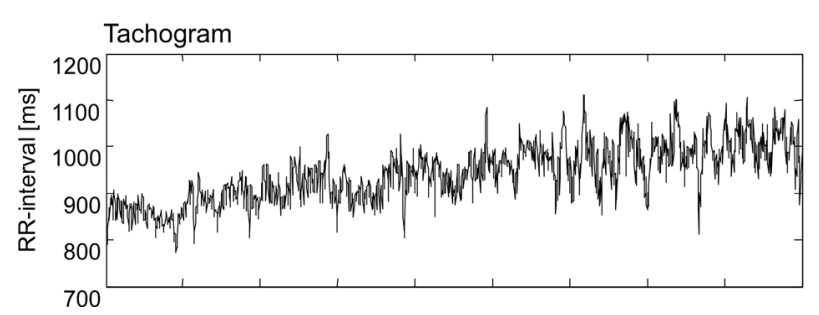

(a)

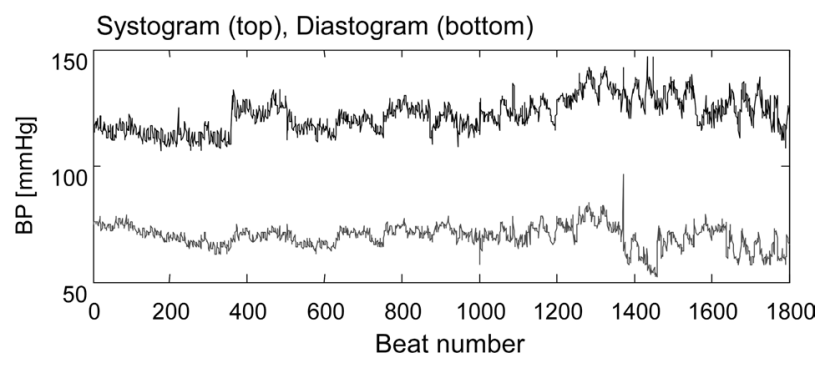

(b)

Fig. 3. Representative example of cardiovascular oscillations: the tachogram - the RR-intervals versus beat number (a), systogram/diastogram - the systolic/diastolic blood pressure values versus beat number (b).

et al., 1996; Task Force Heart Rate Variability, 1996; Szabo et al., 1997]. Moreover, it has been shown that short-term HRV analysis already yields a prognostic value in risk stratification independent of 
that of clinical and functional variables [La Rovere et al., 2003]. However, the underlying regulatory mechanisms are still poorly understood. Hence, we include in our study also the blood pressure variability (BPV). The assessment of BPV from ambulatory blood pressure provides further predictive information of cardiovascular risk but the prognostic value of beat-to-beat BPV needs further investigations [Verdecchia et al., 1999].

The analysis of heart rate or blood pressure variability is often difficult due to a huge amount of artifacts and arrhythmias. While occasional ectopic beats are treated successfully by most preprocessing methods, more complex arrhythmias or arrhythmias which are similar to normal fluctuations may remain untreated. Therefore, we developed a new method for data preprocessing which is presented in the following.

\subsubsection{Preprocessing}

The main objective in the analysis of heart rate and blood pressure is to investigate the cardiovascular system during normal sinus rhythm. Therefore, it is necessary to exclude not only artifacts (e.g. double recognition, i.e. R-peak and T-wave recognized as two beats) but also beats not coming from the sinus node of the heart, so called ventricular premature complexes (VPC). VPCs are not directly controlled by the autonomous nervous system. Practically, this exclusion means a filtering of the time series. The original time series are denoted as the RR-series (derived from the RR-intervals) and the filtered time series as NN-series (normal-tonormal-beat-interval). VPCs in the tachogram are usually characterized by a very short interval followed by a very long RR-interval (ventricular premature beat). Another VPC type is described by a very short interval only (supraventricular premature beat). The 20\%-filter [Haaksma et al., 1995; Kleiger et al., 1987; Zebrowski et al., 1995] considers these facts; if the current value of the tachogram differs more than $20 \%$ from its predecessor, the current value and its successor are marked as not normal. The advantage of this filter is the very simple rule. However, the disadvantages are rarely considered. VPCs with less than $20 \%$ difference are not removed from the series and may falsify almost all HRV or BPV parameters. The RR-intervals recognized as not normal are treated in different ways: either they are simply removed from the series or linear or spline interpolated [Lippman et al., 1994;
Berntson et al., 1990]. The disadvantage of simply removing the beats is the loss of time dependence which can cause the estimation of artificial frequencies. Interpolating linearly may lead to false decreased variabilities, interpolating with splines often fails in time series with many VPCs.

In the following our adaptive filtering algorithm is presented [Wessel et al., 2000b], which is based on our previous interval filter described in [Wessel et al., 1994]. The main advantage of this procedure is the spontaneous adaptation to variability changes in the series, which enables a more reliable removal of artifacts and VPCs. This new filtering algorithm consists of three subprocedures: (i) the removal of obvious recognition errors, (ii) the adaptive percentfilter, and (iii) the adaptive controlling filter.

(i) Obvious misrecognitions are RR-intervals of length zero, BBIs less than $200 \mathrm{~ms}$ (human refractory time) and pauses, i.e. when the heart does not pump for a certain time. These pauses were evaluated with every clinical Holter system, therefore we do not consider them.

(ii) An adaptive filtering procedure was developed based on the adaptive mean value $\mu_{a}$ and the adaptive standard deviation $\sigma_{a}$. Firstly, to estimate the basic variability in the series, a binomial-7-filtered series is calculated. Given the tachogram $x_{1}, x_{2}, \ldots, x_{N}$, the binomial filtered series is given by

$$
t_{n}:=\frac{\begin{array}{c}
x_{n-3}+6 x_{n-2}+15 x_{n-1}+20 x_{n} \\
+15 x_{n+1}+6 x_{n+2}+x_{n+3}
\end{array}}{7} .
$$

The filtered series $t_{1}, t_{2}, \ldots, t_{N}$ reflects the global behavior of sinus node activity without the influence of artifacts and VPCs. The adaptive mean value $\mu_{a}(n)$ and the adaptive standard deviation $\sigma_{a}(n)$ of the binomial filtered series $t_{1}, t_{2}, \ldots, t_{N}$ are defined as:

$$
\begin{gathered}
\mu_{a}(n):=\mu_{a}(n-1)-c \cdot\left(\mu_{a}(n-1)-t_{n}\right), \\
\sigma_{a}(n):=\sqrt{\mu_{a}(n)^{2}-\lambda_{a}(n)},
\end{gathered}
$$

where $c$ is a controlling coefficient $(c \in[0,1])$ and $\lambda_{a}(n)$ is the adaptive second moment

$$
\begin{array}{r}
\lambda_{a}(n):=\lambda_{a}(n-1)-c \cdot\left(\lambda_{a}(n-1)-t_{n}^{2}\right), \\
n=1, \ldots, N .
\end{array}
$$

The exclusion rule of this filter reads as follows: The RR-interval $x_{n}$ is classified as not normal, if

$$
\left|x_{n}-x_{n-1}\right|>\frac{p}{100} x_{n-1}+c_{f} \cdot \bar{\sigma}_{a}
$$


and

$$
\left|x_{n}-x_{l v}\right|>\frac{p}{100} x_{l v}+c_{f} \cdot \bar{\sigma}_{a},
$$

where $p$ is a proportional limit (here $p=10 \%$ ), ' $c_{f} \cdot \bar{\sigma}_{a}$ ' is a generalized $3 \sigma$-rule $\left(c_{f}=3.0\right), x_{l v}$ is the last valid RR-interval and $\bar{\sigma}_{a}$ is the averaged $\sigma_{a}$. Values recognized as not normal are replaced with a random number from $\left[\mu_{a}(n)-(1 / 2) \sigma_{a}(n)\right.$, $\left.\mu_{a}(n)+(1 / 2) \sigma_{a}(n)\right]$ to avoid false decreased variabilities.

(iii) Finally, as a precaution, follows the adaptive controlling procedure. From the resulting time series $x_{1}^{\%}, x_{2}^{\%}, x_{3}^{\%}, \ldots$ of the adaptive percent-filter again the binomial filtered series and the respective adaptive mean value and standard deviation are calculated. The value $x_{n}^{\%}$ is considered to be not normal if

$$
\left|x_{n}^{\%}-\mu_{a}(n)\right|>c_{f_{1}} \cdot \sigma_{a}(n)+\sigma_{b}
$$

where $c_{f_{1}}$ is the filter coefficient (here $c_{f_{1}}=3.0$ ) and $\sigma_{b}$ stands for a basic variability (for HRV here $\sigma_{b}=20 \mathrm{~ms}$ ). This basic variability $\sigma_{b}$ was introduced to reduce filtering errors for time series with low variability (near the accuracy of RR-interval detection). Not normal values are replaced with the respective values of the binomial filtered series. The advantage of our newly introduced adaptive filtering procedure is the spontaneous adaptation of the filter coefficients due to sudden changes in the series (e.g. sudden heart rate increase, see Fig. 4). The choice of a generalized $3 \sigma$-rule in this algorithm was done heuristically and we got excellent filtering

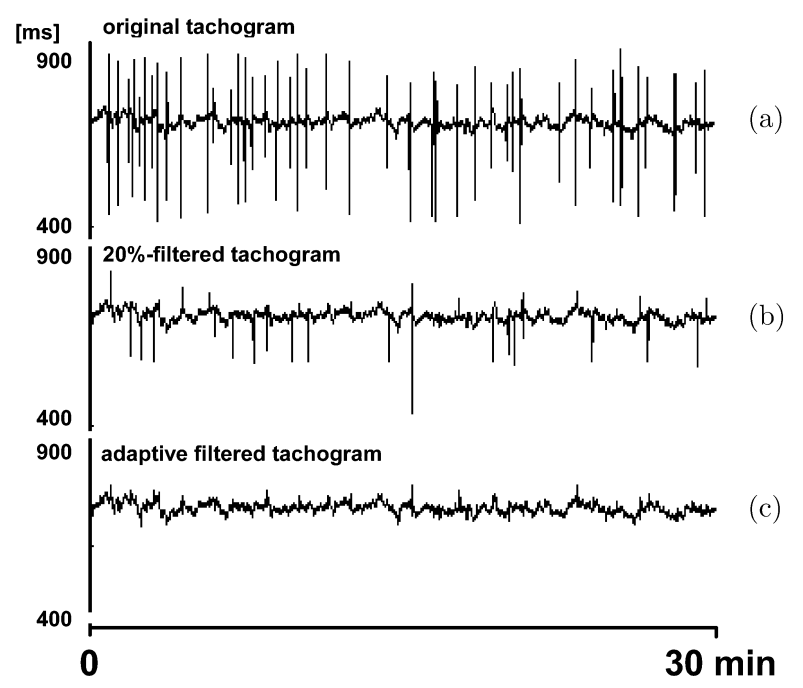

Fig. 4. One typical HRV series including VPCs with different prematureness (a), the results after filtering using the $20 \%$ rule (b) and the filtered time series after adaptive preprocessing (c). results for most of the data. It has to be mentioned here, that we analyzed thousands of human and animal time series so far. Small changes of the controlling parameters will not significantly influence the filtering result. A MATLAB implementation of the preprocessing algorithm is available from tocsy.agnld.uni-potsdam.de.

\subsubsection{Time and frequency domain parameter}

Standard methods of HRV analysis include time and frequency domain parameters; these are linear methods. Time domain parameters are based on simple statistical methods derived from the RR-intervals as well as the differences between them. Mean heart rate is the simplest parameter, but the standard deviation over the whole time series ( $\mathrm{sdNN}$ ) is the most prominent HRV measure for estimating overall HRV. A list of these parameters is given in Table 1 . These parameters can be calculated for short (5 minutes) and long (24 hours) term epochs, representing short-term resp. longterm variability or for averaged short term epochs (e.g. mean of 288 five-minutes intervals a day). The overall HRV estimate sdNN and other time domain parameters can be used to predict mortality in the recovering period after an myocardial infarction. In one of the first risk studies using these parameters, Kleiger et al. [1987] showed that an $s d N N<50 \mathrm{~ms}$ was associated with a 5.3-fold increased mortality when compared to patients with preserved HRV $(s d N N>100 \mathrm{~ms})$.

Time domain geometrical methods (see Table 1) are methods where the BBI are converted into special geometrical forms quantifying their distribution. Special forms are used to make the approach more insensitive to artifacts and ectopic beats. A disadvantage of these methods is that they require a considerable number of RR-intervals, thus they are not applicable to very short-term time series. A triangular index HRVi showing reduced HRV has been associated with both arrhythmic and nonarrhythmic death [Task Force Heart Rate Variability, 1996; Hartikainen et al., 1996]. We introduced a more robust method to quantify the distribution [Voss et al., 1996b] based on information theory, in particular the method uses the Shannon- and the Renyi-entropy of the histogram. We demonstrated the usefulness for risk stratification two years later in a blinded study [Voss et al., 1998]. 
Table 1. Description of time- and frequency domain parameters, standards [Task Force Heart Rate Variability, 1996] and additional measures we developed $(\bullet)$. BBI here stands for the filtered beat-to-beat intervals (NN-intervals).

\begin{tabular}{|c|c|c|}
\hline Variable & Units & Definition \\
\hline \multicolumn{3}{|c|}{ Time domain statistical methods } \\
\hline meanNN & $\mathrm{ms} / \mathrm{mmHg}$ & Mean BBI resp. mean BP \\
\hline sdNN & $\mathrm{ms} / \mathrm{mmHg}$ & Standard deviation of all BBI resp. BP values \\
\hline sdaNN5 & $\mathrm{ms}$ & $\begin{array}{l}\text { Standard deviation of successive five minutes BBI averages } \\
\text { (for HRV only) }\end{array}$ \\
\hline rmssd & $\mathrm{ms} / \mathrm{mmHg}$ & Root mean square of successive BBI/BP differences \\
\hline pNN50 & $\%$ & $\begin{array}{l}\text { Percentage of NN-interval differences greater than } 50 \mathrm{~ms} \\
\text { (for HRV only) }\end{array}$ \\
\hline - $\mathrm{pNNX}$ & $\%$ & $\begin{array}{l}\text { Percentage of beat-to-beat differences greater } \\
\text { than Xms } / \mathrm{mmHg} \text { (e.g. } X=100 / 200 \mathrm{~ms}, X=5 / 10 \mathrm{mmHg})\end{array}$ \\
\hline - $\mathrm{pNNIX}$ & $\%$ & $\begin{array}{l}\text { Percentage of beat-to-beat differences lower } \\
\text { than Xms } / \mathrm{mmHg} \text { (e.g. } X=10 / 20 / 30 \mathrm{~ms}, X=3 / 6 \mathrm{mmHg})\end{array}$ \\
\hline • Shannon & none & $\begin{array}{l}\text { Shannon entropy of the histogram } \\
\text { (density distribution of the BBIs/BP values) }\end{array}$ \\
\hline - RenyiX & none & $\begin{array}{l}\text { Renyi entropy of order } X \text { of the histogram } \\
\quad(\text { e.g. } X=2 / 4 / 0.25)\end{array}$ \\
\hline \multicolumn{3}{|c|}{ Time domain geometrical methods } \\
\hline HRVi & none & HRV triangular index \\
\hline TINN & $\mathrm{ms}$ & Baseline width of the minimum square difference triangle \\
\hline \multicolumn{3}{|c|}{ Frequency domain methods } \\
\hline $\mathrm{P}$ & $\mathrm{ms}^{2} / \mathrm{mmHg}^{2}$ & Total power from $0-0.4 \mathrm{~Hz}$ \\
\hline ULF & $\mathrm{ms}^{2} / \mathrm{mmHg}^{2}$ & Ultra low frequency band $0-0.0033 \mathrm{~Hz}$ \\
\hline VLF & $\mathrm{ms}^{2} / \mathrm{mmHg}^{2}$ & Very low frequency band $0.0033-0-0.4 \mathrm{~Hz}$ \\
\hline $\mathrm{LF}$ & $\mathrm{ms}^{2} / \mathrm{mmHg}^{2}$ & Low frequency band $0.04-0.15 \mathrm{~Hz}$ \\
\hline $\mathrm{HF}$ & $\mathrm{ms}^{2} / \mathrm{mmHg}^{2}$ & High frequency band $0.15-0.4 \mathrm{~Hz}$ \\
\hline $\mathrm{LF} / \mathrm{HF}$ & none & Quotient of LF and HF \\
\hline LFn & none & Normalized low frequency band $(\mathrm{LF} /(\mathrm{LF}+\mathrm{HF}))$ \\
\hline
\end{tabular}

Frequency domain HRV parameters enable one to analyze periodic dynamics in the heart rate time series [Akselrod et al., 1981]. There are mainly two different techniques for spectral analysis: methods based on Fast Fourier Transform (FFT) and parametric autoregressive model estimations of wavelet approaches. The results using different spectral methods however should be comparable (apart from differences in time and frequency resolution). The Task Force on HRV [Task Force Heart Rate Variability, 1996] recommended that power spectral analysis of 5-minute ECG recordings should be used to assess autonomic physiology and pharmacology. Very low, low and high frequencies (see Table 1) can be estimated from such 5-minute ECG recording. The high frequency power reflects modulation of vagal activity by respiration whereas the low frequency power represents vagal and sympathetic activity via the baroreflex loop. The low-to-high frequency ratio is used as an index of sympathovagal balance [Malliani et al., 1991]. The capability of frequency domain parameters for risk stratification of post-infarction patients was proven by Bigger et al. [1992] — a reduction in the ultra low and very low frequency power is associated with pathologies.

For blood pressure series all described HRV parameters can be accordingly calculated, only some statistical parameters need to be adapted (e.g. pNN50 makes no sense for BPV - the standard deviation for $\mathrm{BP}$ series differs from 5 to $10 \mathrm{mmHg}$ ).

\subsection{Nonlinear analysis of heart rate and blood pressure variability}

Heart rate and blood pressure variability reflect the complex interactions of many different control loops of the cardiovascular system. In relation to the complexity of the sinus node activity modulation system, a predominantly nonlinear behavior has to be assumed. Thus the detailed description and classification of dynamic changes using time and frequency measures is often not sufficient. Therefore we introduce in the following subsection nonlinear methods based on symbolic dynamics, renormalized 
entropy, finite time growth rates, recurrence quantification analysis, large-scale dimension densities as well as further nonlinear approaches developed by other groups. In the application section we compare the nonlinear measures with each other, and also with the linear parameters introduced in Sec. 2.2.2.

\subsubsection{Symbolic dynamics}

We have shown already in 1995 that symbolic dynamics is an efficient approach to analyze dynamic aspects of HRV [Kurths et al., 1995; Voss et al., 1996b]. The first step in this analysis is the transformation of the time series into symbol sequences with symbols from a given alphabet. Some detailed information is lost in this process, but the coarse dynamic behavior can be analyzed (see Fig. 5) [Engbert et al., 1997]. Wackerbauer et al. [1994] used the methodology of symbolic dynamics for the analysis of the logistic map, where a generic partition is known. However, for physiological time series analysis a more pragmatic approach is necessary. The transformations into symbols have to be chosen context-dependent. For this reason, we develop complexity measures on the basis of such context-dependent transformations, which have a close connection to physiological phenomena and are relatively easy to interpret.

Comparing different kinds of symbol transformations, we found that the use of four symbols, as explained in Eq. (8), is appropriate for our purpose.

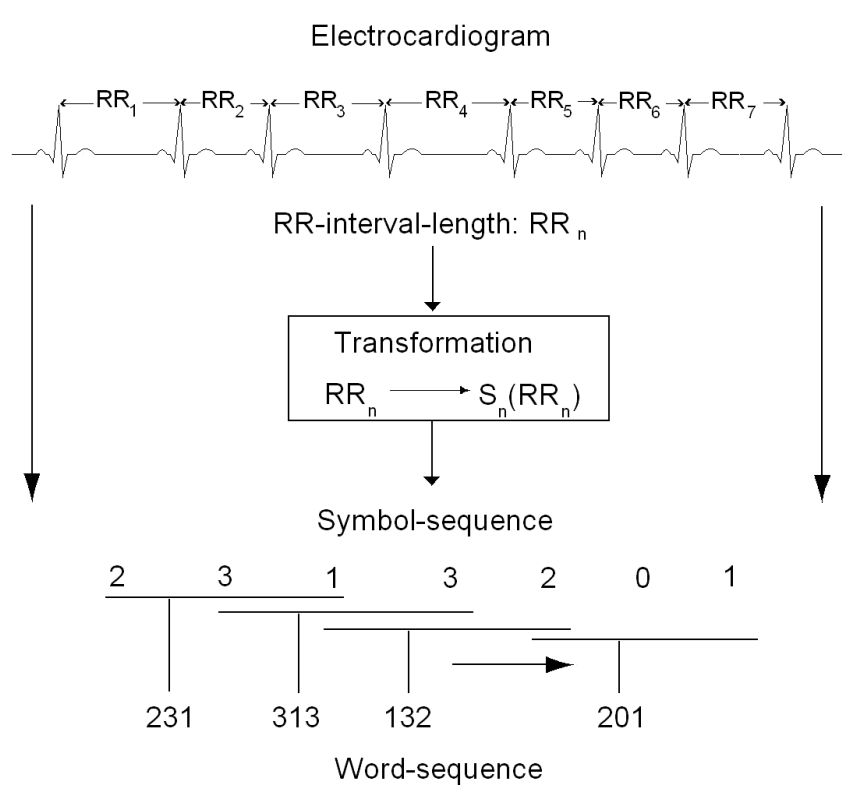

Fig. 5. Sketch of symbolic dynamic transformation.
The time series $x_{1}, x_{2}, x_{3}, \ldots, x_{N}$ is transformed into the symbol sequence $s_{1}, s_{2}, s_{3}, \ldots, s_{N}, s_{i} \in A$ on the basis of the alphabet $A=\{0,1,2,3\}$.

$$
s_{i}\left(x_{i}\right)=\left\{\begin{array}{l}
0 \quad \mu \quad<\quad<x_{i} \leq(1+a) \cdot \mu \\
1:(1+a) \cdot \mu<x_{i}<\infty \\
2:(1-a) \cdot \mu<x_{i} \leq \mu \\
3: \quad 0 \quad<x_{i} \leq(1-a) \cdot \mu \\
i=1,2,3, \ldots
\end{array}\right.
$$

The transformation into symbols refers to three given levels where $\mu$ denotes the mean beat-to-beat interval and $a$ is a special parameter that we have chosen 0.05 . We tested several values of $a$ from 0.02 to 0.08 , however the resulting symbol sequences differed not significantly. Figure 6 gives some examples for this dependence: for $a=0.5$ and $a=0.6$ the word distributions nearly coincide — only for higher values $(a=0.8)$ we see some differences for the subjects (3) and (4).

There are several quantities that characterize such symbol strings. In this study we analyze the frequency distribution of length three words, i.e. substrings which consist of three symbols from the alphabet $A$ (Fig. 5, bottom), leading to maximum 64 different words (bins). This is a compromise of having, on the one hand, some dynamical information and of having, on the other hand, a sufficient good statistics to estimate the probability distribution (cf. Fig. 6). We consider the following measures of complexity:

(i) The Shannon entropy $H_{k}$ ("fwshannon") calculated from the distribution $p$ of words is the classic measure for complexity in time series:

$$
H_{k}=-\sum_{\omega \in W^{k}, p(\omega)>0} p(\omega) \log p(\omega),
$$

where $W^{k}$ is the set of all words of length $k$. Larger values of Shannon entropy refer to higher complexity in the corresponding tachograms and lower values to lower ones.

(ii) Next, we count the "forbidden words" in the distribution of words with length three - that are the number of words which never or only seldomly occur. A high number of forbidden words stands for a rather regular behavior in the time series. If the time series is highly complex in the sense of Shannon, only a few forbidden words will be found.

(iii) To measure especially low variability, we introduce the parameter "POLVAR20". In this way 

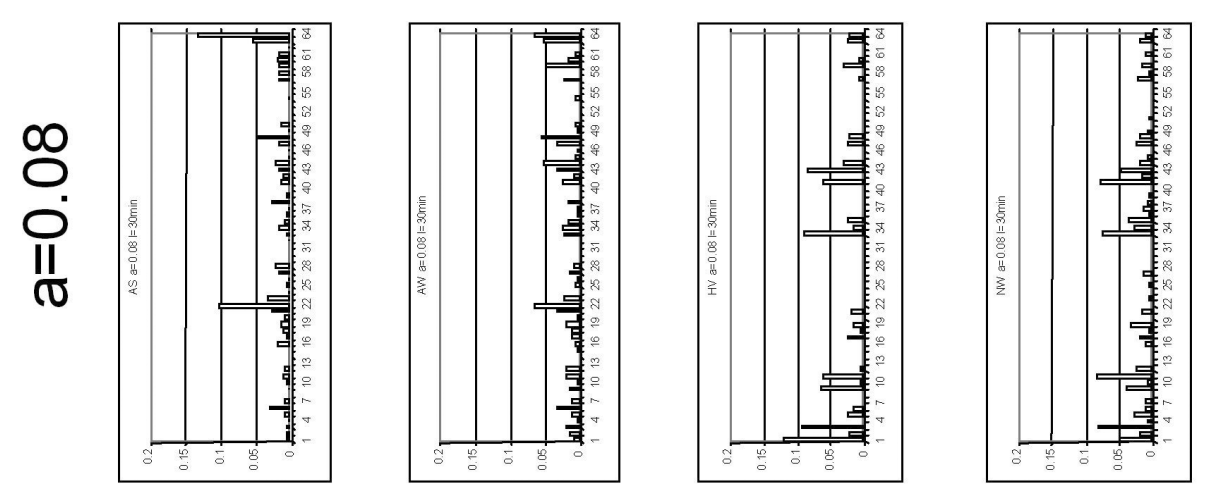

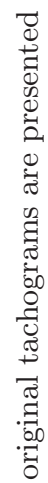
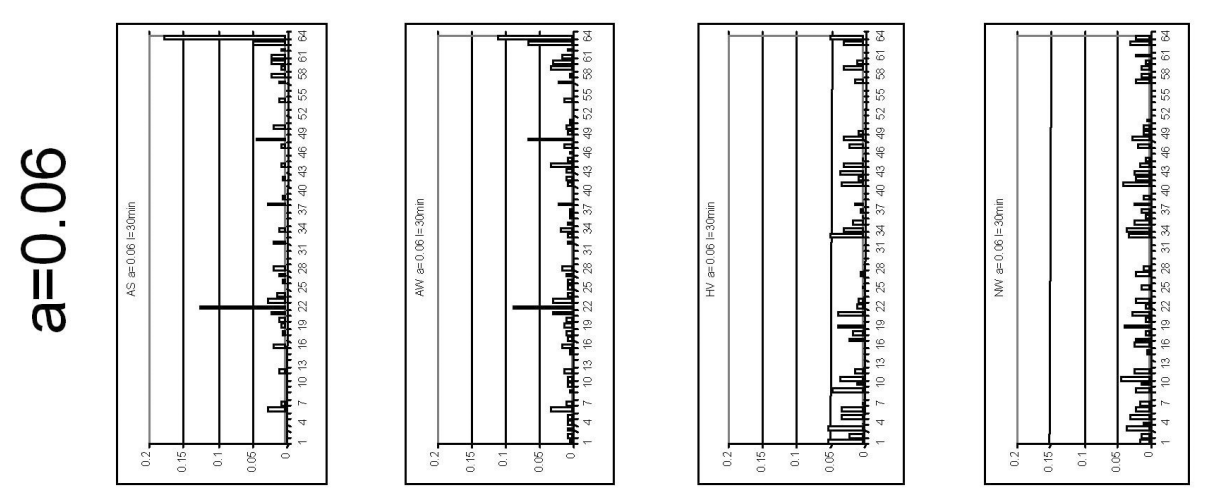

0

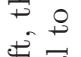

营

\&

ธี क.

㐫

所華

$\underbrace{\infty}_{\approx}: \stackrel{\infty}{:}$

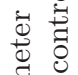

ซ్ర
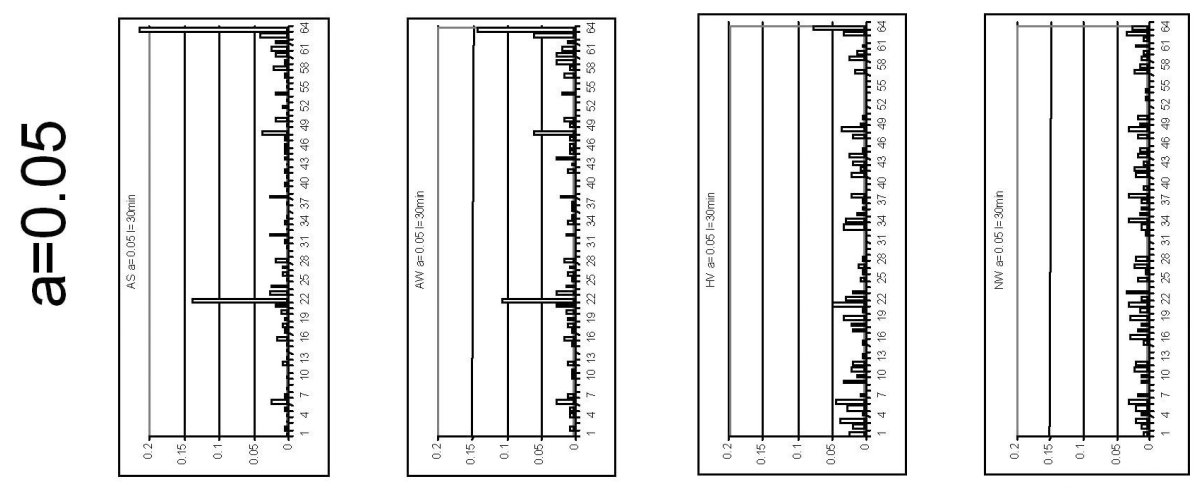

ํ. 유

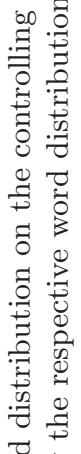

흉 으

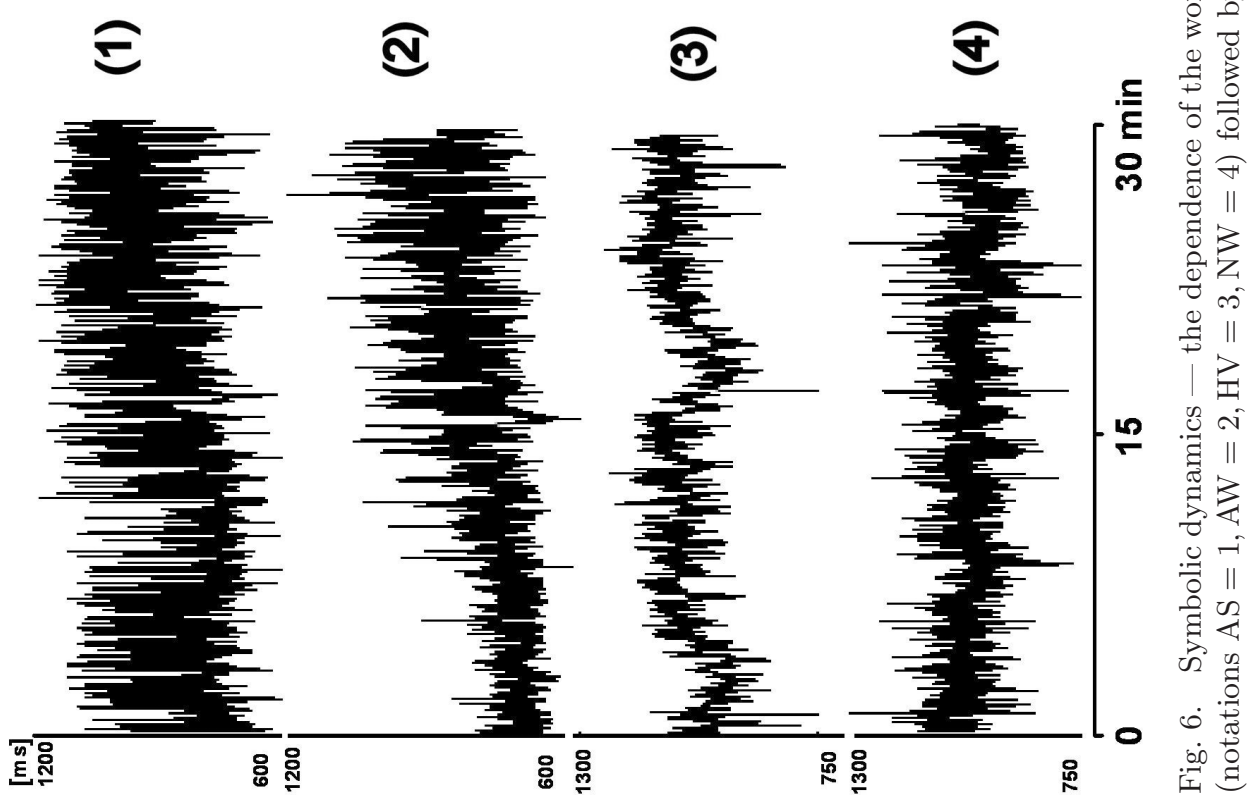


successive symbols of another simplified alphabet, consisting only of symbols " 0 " and " 1 ", were analyzed. Here the symbol " 0 " stands for a small difference between two successive beats, whereas " 1 " represent those cases where the difference between two successive beats exceeds this special limit:

$$
s_{n}=\left\{\begin{array}{l}
1:\left|x_{n}-x_{n-1}\right| \geq 20 \mathrm{~ms} \\
0:\left|x_{n}-x_{n-1}\right|<20 \mathrm{~ms}
\end{array}\right.
$$

Words consisting only of an unique type of symbols (either all "0" or all "1") were counted. To get a statistically appropriate estimate of the word distribution, we choose words of length six, where again at maximum 64 different types of words can occur. "POLVAR20" represents the probability of the word type "000000" occurrence and is able to detect even intermittent decreased variability. Note that the special limit of $20 \mathrm{~ms}$ can be adapted to other values if necessary, for instance in animal studies.

\subsubsection{Renormalized entropy}

Another approach which might be capable of assessing complex properties of cardiac periodograms is the "renormalized entropy". The fundamental idea is to determine the complexity of cardiac periodograms related to a fixed reference. Based on general considerations in thermodynamics, Klimontovich suggested to compare the relative degree of order of two different distributions by renormalizing the reference distribution to a given energy. Saparin [Saparin et al., 1994] proposed a procedure for calculating this quantity from time series and applied it to the logistic map. Applications of renormalized entropy to physiological data were previously introduced [Kurths et al., 1995; Voss et al., 1996b; Wessel et al., 1994; Kopitzki et al., 1998]. That method, however, suffers from a potential lack of reproducibility for heart rate data. Therefore, we developed another method for the computation of renormalized entropy $\mathrm{RE}_{\mathrm{AR}}$ based on an autoregressive spectral estimation [Wessel et al., 2000b]. In the following two paragraphs our new method is briefly introduced.

To compare the relative degree of order of two different distributions, the reference distribution is renormalized to a given energy. The complexity of any distribution in relation to a fixed reference distribution is estimated by solving an integral equation. Considering two tachograms (time series of beat-to-beat intervals) with the density distribution estimates $f_{0}(x)$ and $f_{1}(x)$ and using the estimate $f_{0}(x)$ as a reference, the renormalized density distribution $\bar{f}_{0}(x)$ of $f_{0}(x)$ is defined as:

$$
\bar{f}_{0}(x):=\frac{f_{0}(x)^{T}}{\int f_{0}(x)^{T} d x},
$$

where $T$ is the solution of the integral equation

$$
\int \ln f_{0}(x)^{\left(\bar{f}_{0}(x)-f_{1}(x)\right)} d x=0,
$$

The solution of (12) has to be determined numerically. The renormalized entropy $\mathrm{RE}$ of the distribution $f_{1}(x)$ is defined by the following interchanging algorithm involving the Shannon-entropy of distribution $f(x)$ defined by

$$
S(f(x))=-\int f(x) \cdot \ln f(x) d x
$$

Procedure:

- Calculation of $\Delta_{1}=S\left(f_{1}(x)\right)-S\left(\bar{f}_{0}(x)\right)$ with the distribution $f_{0}(x)$ as the reference $\left(f_{0}(x)\right.$ is renormalized). The value of $T$ is denoted $T_{1}=T$.

- Calculation of $\Delta_{2}=S\left(f_{0}(x)\right)-S\left(\bar{f}_{1}(x)\right)$ with the distribution $f_{1}(x)$ as the reference $\left(f_{1}(x)\right.$ is renormalized). The resulting $T$ value is denoted $T_{2}=T$.

- If $T_{1}>T_{2}$, the distribution $f_{0}(x)$ is found to be the more disordered one (in the sense of renormalized entropy - i.s.r.e.) and the renormalized entropy $\mathrm{RE}$ is defined as $\mathrm{RE}=\Delta_{1}$. Otherwise $f_{1}(x)$ is the more disordered distribution (i.s.r.e.) and the renormalized entropy is $\mathrm{RE}=-\Delta_{2}$.

Calculation of the renormalized entropy requires estimating the tachogram distributions. Here we use an autoregressive spectral estimation of the filtered and interpolated tachogram. A known problem of autoregressive spectral estimations is the bias which might appear even in idealized circumstances. To overcome this problem, a sinusoidal oscillation with a fixed amplitude and frequency was added to the time series. The amplitude of $40 \mathrm{~ms}$ was chosen to obtain a dominant peak in the spectral estimation and the frequency was set to $0.4 \mathrm{~Hz}$, which is the upper limit of the high frequency band [Task Force Heart Rate Variability, 1996] (Fig. 7). A spectral density estimation in the interval $[0,0.42] \mathrm{Hz}$ was used to include all physiological modulations and the calibration peak. Using 


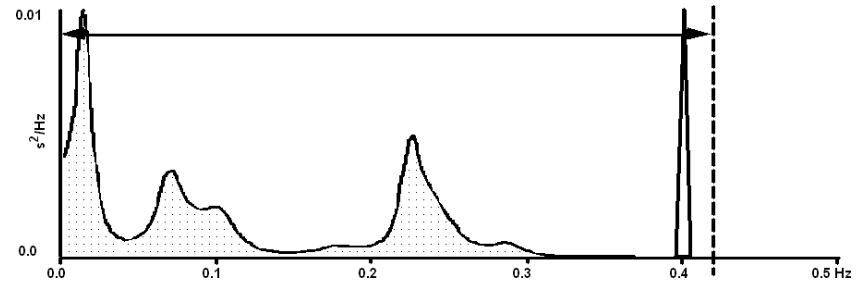

Fig. 7. Estimation of the tachogram distribution is the basis for calculating renormalized entropy. Here, the autoregressive spectral distribution (dotted area) together with the calibration peak at $0.4 \mathrm{~Hz}$ was used. Given a fixed reference distribution the complexity of all distributions which should be analyzed will be determined relative to this reference.

a reference tachogram from a healthy subject with normal low and high frequency modulations, the $\mathrm{RE}_{\mathrm{AR}}$ method is designed so that either a decreased HRV or a pathological spectrum leads to positive values of the renormalized entropy.

\subsubsection{Finite-time growth rates}

Lyapunov exponents of a dynamical system reflect average effective growth rates of infinitesimal uncertainties over an infinite duration. However, time series analysis is restricted to the analysis of finite time series and thus it is difficult to estimate Lyapunov exponents [Oseledec, 1968; Wolf et al., 1985; Eckmann et al., 1986; Kurths \& Herzel, 1987; Smith, 1994]. Moreover, often we only have rather short time series which do not allow estimating Lyapunov exponents. Therefore, we concentrate on quantifying the state-dependent short-term predictability through finite-time growth rates. Note that these differ from the finite-time Lyapunov exponents defined in [Lorenz, 1965; Abarbanel et al., 1991; Brown et al., 1991; Yoden \& Nomura, 1993; Ziehmann et al., 1999] as well as the finitetime growth rates of [Nese, 1989], both of which require the knowledge of the tangent maps thus the equations governing the dynamics. In [Ziehmann et al., 1999] we have discussed the significant differences between these quantities.

Our finite-time growth rates are approximations based on the idea of Wolf et al. [1985] and can be introduced in three steps.

(i) Firstly, pseudo phase spaces of the system are constructed using delay coordinates [Takens, 1981]. Their dimension is denoted by $n$ and the fixed delay by $\tau$.

(ii) Next, for each point in this constructed phase space $I_{k}=\left[x_{k}, x_{k+\tau}, \ldots, x_{k+(n-1) \cdot \tau}\right], k=$ $1, \ldots,(N-(n-1) \cdot \tau)$ of the measured tachogram
$I=\left[x_{1}, x_{2}, \ldots, x_{N}\right]$ the nearest neighbor $\bar{I}_{k}$ is determined. $\bar{I}_{k}$ is defined as that state which has the minimal Euclidean distance to the $I_{k}$. $\left\|I_{u}-I_{v}\right\|$ denotes the Euclidean distance of the state $I_{u}$ to $I_{v}$, i.e.

$$
\left\|I_{u}-I_{v}\right\|=\sqrt{\sum_{j=0}^{n-1}\left(x_{u+j \cdot \tau}-x_{v+j \cdot \tau}\right)^{2}} .
$$

Then the minimal distance $d_{k}$ to the state $I_{k}$ is given by

$$
\begin{aligned}
d_{k}= & \min _{\substack{i=1, \ldots, N-(n-1) \cdot \tau \\
|i-k|>(n-1) \cdot \tau}}\left\|I_{k}-I_{i}\right\|, \\
& k=1, \ldots, N-(n-1) \cdot \tau
\end{aligned}
$$

and the nearest neighbor by

$$
\begin{aligned}
\bar{I}_{k}= & \left\{I_{m} \mid\left\|I_{k}-I_{m}\right\|=d_{k}\right\}, \\
& k=1, \ldots, N-(n-1) \cdot \tau .
\end{aligned}
$$

Note that the time lag of the nearest neighbor to the base point has to be at least one window length, i.e. $|i-k|>(n-1) \cdot \tau$ and we only consider points as neighbors if their distance to the base point is less than $10 \%$ of the attractor diameter (maximum distance between any two points in the series).

(iii) Next, we analyze the evolution of the states $I_{k}$ and $\bar{I}_{k}$ during the time $T$. After these $T$ steps we get the states $I_{k}^{T}=\left[x_{k+T}, x_{k+T+\tau}, \ldots\right.$, $\left.x_{k+T+(n-1) \cdot \tau}\right]$ and $\bar{I}_{k}^{T}$, respectively. The distance between both states $\left\|I_{k}^{T}-\bar{I}_{k}^{T}\right\|$ represents the divergence after $T$ evolution steps. From the original distance of both states and the distance after $T$ steps we calculate the finite-time growth rate $\lambda_{k}^{(n, \tau, T)}$ :

$$
\begin{aligned}
\lambda_{k}^{(n, \tau, T)} & =\frac{1}{T} \ln \frac{\left\|I_{k}^{T}-\bar{I}_{k}^{T}\right\|}{\left\|I_{k}-\bar{I}_{k}\right\|}, \\
k & =1, \ldots, N-(n-1) \cdot \tau .
\end{aligned}
$$

$\lambda_{k}^{(n, \tau, T)}$ quantifies the local short-term predictability at the point $I_{k}$. If these $\lambda_{k}^{(n, \tau, T)}>0$, the distance after the evolution time $T$ increases; otherwise, it decreases.

We calculate the finite-time growth rates for each point of the delay phase space, which leads to a time series of growth rates $\lambda_{k}^{(n, \tau, T)}$. Its average, the average growth rate $\lambda^{(n, \tau, T)}$,

$$
\lambda^{(n, \tau, T)}=\frac{1}{N-(n-1) \cdot \tau+1} \sum_{k=1}^{N-(n-1) \cdot \tau} \lambda_{k}^{(n, \tau, T)}
$$

quantifies a global short-term predictability. 
For the analysis of HRV data we selected the following controlling parameters. The dimension $n$, i.e. the length of the selected tachogram parts varied from 3 to 9 . We choose this range to cover an interval up to 9, which is a typical order of an autoregressive model for short-term HRV tachograms [Wessel et al., 1994]. The evolution time and the delay are defined as $T=1,2,3$ and $\tau=1,2,3$ respectively.

Additionally, to reduce random influences, we consider a three and a five nearest neighbor approach. According to Eq. (16) we determine the five nearest neighbors $\bar{I}_{k}^{1}, \ldots, \bar{I}_{k}^{5}$ of the point $I_{k}$ and evolve all neighbors over the evolution time $T$. The finite-time growth rates for the three and the five nearest neighbor approach are derived from the average distances before and after the evolution time.

\subsubsection{Recurrence quantification analysis}

The method of recurrence plots (RP) was firstly introduced to visualize the time dependent behavior of a system's dynamics, which can be pictured as a trajectory $\mathbf{x}_{i} \in \mathcal{R}^{n}(i=1, \ldots, N)$ in the $n$-dimensional phase space [Eckmann et al., 1987]. It represents the recurrence of the phase space trajectory to a certain state, which is a fundamental property of deterministic dynamical systems [Argyris et al., 1994; Ott, 1993]. The main step of this visualization is the calculation of the $N \times N$-matrix

$$
\mathbf{R}_{i, j}:=\Theta\left(\varepsilon_{i}-\left\|\mathbf{x}_{i}-\mathbf{x}_{j}\right\|\right), \quad i, j=1, \ldots, N,
$$

where $\varepsilon_{i}$ is a cut-off distance, $\|\cdot\|$ a norm (e.g. the Euclidean norm) and $\Theta(x)$ the Heaviside function. Analogous to the last section (3), the phase space vectors for one-dimensional time series $u_{i}$ from observations can be reconstructed by using the Takens time delay method $\mathbf{x}_{i}=\left(u_{i}, u_{i+\tau}, \ldots\right.$, $u_{i+(m-1) \tau}$ ) [Kantz \& Schreiber, 1997]. The dimension $m$ can be estimated with the method of false nearest neighbors (theoretically, $m=2 n+1$ ) [Argyris et al., 1994; Kantz \& Schreiber, 1997]. The cut-off distance $\varepsilon_{i}$ defines a sphere centered at $\mathbf{x}_{i}$. If $\mathbf{x}_{j}$ falls within this sphere, the state will be close to $\mathbf{x}_{i}$ and thus $\mathbf{R}_{i, j}=1$. These $\varepsilon_{i}$ can be either constant for all $\mathbf{x}_{i}$ [Koebbe \& Mayer-Kress, 1992] or they can vary in such a way, that the sphere contains a predefined number of close states [Eckmann et al., 1987]. We use a fixed $\varepsilon_{i}$ and the Euclidean norm resulting in a symmetric RP. The binary values in $R_{i, j}$ can be simply visualized by a matrix plot with the colors black (1) and white (0).
The recurrence plot exhibits characteristic large-scale and small-scale patterns which are caused by typical dynamical behavior [Eckmann et al., 1987; Webber \& Zbilut, 1994], e.g. diagonals (similar local evolution of different parts of the trajectory) or horizontal and vertical black lines (state does not change for some time).

Zbilut and Webber developed the recurrence quantification analysis (RQA) to quantify an RP [Trulla et al., 1996; Webber \& Zbilut, 1994; Zbilut \& Webber, Jr., 1992]. They define measures using the recurrence point density and the diagonal structures in the recurrence plot, the recurrence rate, the determinism, the maximal length of diagonal structures, the entropy and the trend. A computation of these measures in small windows moving along the main diagonal of the RP yields the time dependent behavior of these variables and, thus, makes the identification of transitions in the time series possible [Trulla et al., 1996].

The RQA measures are mostly based on the distribution of the length of the diagonal structures in the RP. Additional information about further geometrical structures, such as vertical and horizontal elements, are not included there. Gao has therefore recently introduced a recurrence time statistics, which corresponds to vertical structures in an RP [Gao, 1999; Gao \& Cai, 2000]. In Fig. 8 an example of a recurrence plot for the logistic map is given recurrent states are characterized by black rectangles in this plot.

In the following, we extend this view on the vertical structures and define measures of complexity based on the distribution of the vertical line length. Since we are using symmetric RPs here, it suffices to only consider the vertical structures.

We consider a point $\mathbf{x}_{i}$ of the trajectory and the set of its associated recurrence points $S_{i}:=\left\{\mathbf{x}_{k}\right.$ : $\left.\mathbf{R}_{i, k} \stackrel{!}{=} 1 ; k \in[1 \cdots N-1]\right\}$. Denote a subset of these recurrence points $s_{i}:=\left\{\mathbf{x}_{l} \in S_{i}:\left(\mathbf{R}_{i, l}\right.\right.$. $\left.\mathbf{R}_{i, l+1}\right)+\left(\mathbf{R}_{i, l} \cdot \mathbf{R}_{i, l-1}\right)>0 ; l \in[1 \cdots N], \mathbf{R}_{i, 0}=$ $\left.\mathbf{R}_{i, N+1}:=0\right\}$ which contains the recurrence points forming the vertical structures in the RP at column $i$. In continuous time systems with high time resolution and with a not too small threshold $\varepsilon$, a large part of this set $s_{i}$ usually corresponds to the sojourn points described in [Gao, 1999; Gao \& Cai, 2000]. Next, we determine the length $v$ of all connected subsets $\left\{\mathbf{x}_{j} \notin s_{i} ; \mathbf{x}_{j+1} \ldots \mathbf{x}_{j+v} \in\right.$ $\left.s_{i} ; \mathbf{x}_{j+v+1} \notin s_{i}\right\}$ in $s_{i} \cdot P_{i}(v)=\left\{v_{l} ; l=1,2, \ldots, L\right\}$ denotes the set of all occurring subset lengths in $s_{i}$ and from $\bigcup_{i=1}^{N} P_{i}(v)$ we determine the 


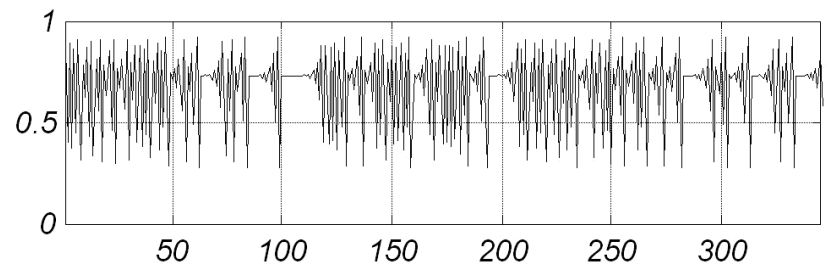

(a)

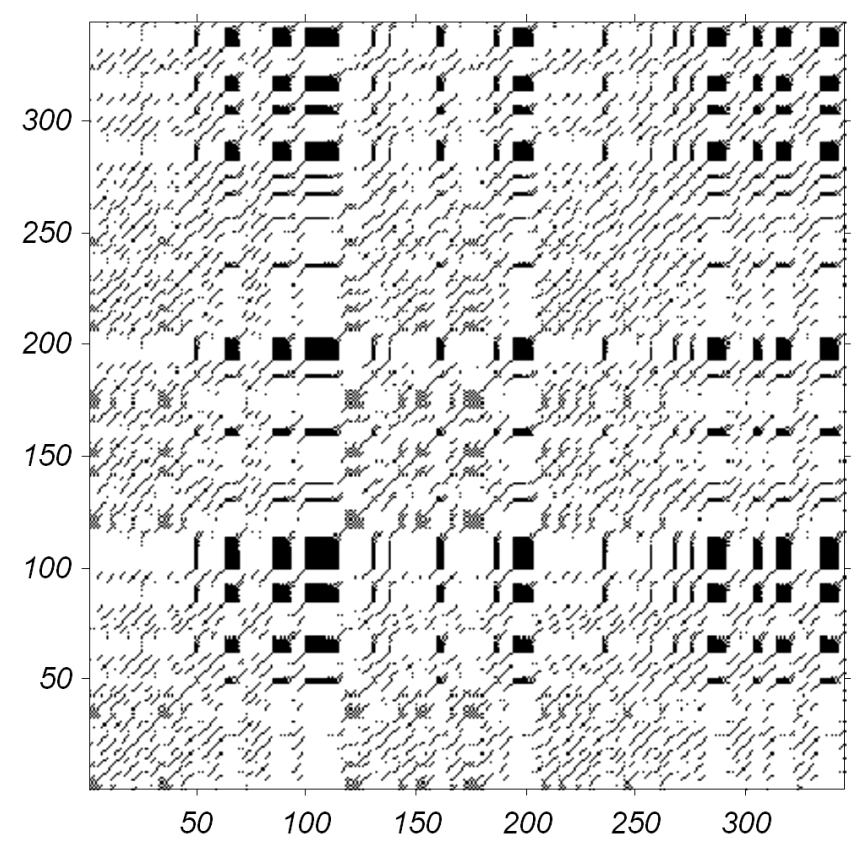

(b)

Fig. 8. Time series of the logistic map $\left(x_{n+1}=a x_{n}\left(1-x_{n}\right)\right)$ for control parameter $a=3.679$ (a) as well as the corresponding recurrence plot (b). Embedding dimension was chosen $m=1$, time delay $\tau=1$ and distance cutoff $\varepsilon=0.1 \sigma$.

distribution of the vertical line lengths $P(v)$ in the entire RP.

Analogous to the definition of the determinism [Webber \& Zbilut, 1994; Marwan, 1999], we compute the ratio between the recurrence points forming the vertical structures and the entire set of recurrence points

$$
\Lambda:=\frac{\sum_{v=v_{\min }}^{N} v P(v)}{\sum_{v=1}^{N} v P(v)},
$$

and call it laminarity $\Lambda$ [Marwan et al., 2002]. The computation of $\Lambda$ is realized for $v$ which exceeds a minimal length $v_{\min }$. For maps we use $v_{\min }=2$. $\Lambda$ is the measure of the amount of vertical structures in the whole RP and represents the occurrence of laminar states in the system, without, however, describing the length of these laminar phases. It will decrease if the RP consists of more single recurrence points than vertical structures.

Next, we define the average length of vertical structures

$$
T:=\frac{\sum_{v=v_{\min }}^{N} v P(v)}{\sum_{v=v_{\min }}^{N} P(v)},
$$

which we call trapping time $T$. The computation also uses the minimal length $v_{\min }$ as in $\Lambda$. The measure $T$ contains information about the amount and the length of the vertical structures in the RP.

Finally, we use the maximal length of the vertical structures in the RP

$$
V_{\max }=\max \left(\left\{v_{l} ; l=1,2, \ldots, L\right\}\right)
$$

as a measure, which is the analogue to the standard RQA measure $L_{\max }$ [Webber \& Zbilut, 1994].

Although the distribution of the diagonal line lengths contains information about the vertical line lengths, the two distributions are significantly different.

For HRV analysis we choose embedding dimensions of $m=3 \ldots 12$, a delay of $\tau=1$ and a distance cutoff of $\varepsilon=50 \ldots 200 \mathrm{~ms}$.

\subsubsection{Large-scale dimension densities}

The large-scale dimension density LASDID [Raab \& Kurths, 2001] is estimated using a normalized Grassberger-Procaccia algorithm, which leads to a suitable correction of systematic errors produced by boundary effects associated with large scales of a system. This allows for an analysis of rather short and nonstationary data.

In order to calculate the correlation dimension $D_{2}$ of a system with the GrassbergerProcaccia algorithm [Grassberger \& Procaccia, 1983], firstly the attractor has to be reconstructed using phase space reconstruction. The embedded time series consists of vectors $\{\mathbf{x}(t)=$ $\left.\left(x_{1}(t), x_{2}(t), \ldots, x_{m}(t)\right)\right\}$, where $m$ is the embedding dimension. Then one has to calculate the correlation integral

$C(r, m)=\frac{1}{N(N-1)} \sum_{i \neq j} \theta\left(r-\left|\mathbf{x}\left(t_{i}\right)-\mathbf{x}\left(t_{j}\right)\right|\right)$

where $\theta$ is the Heaviside function and $r$ is the radius around each point within neighboring points 
are counted for the correlation sum. $D_{2}$ is then defined as

$$
D_{2}=\lim _{r \rightarrow 0} \lim _{m \rightarrow \infty}\left(\frac{\mathrm{d} \log C(r, m)}{(-\mathrm{d} \log (r))}\right),
$$

if this limit exists [Grassberger \& Procaccia, 1983]. Because it is impossible to reach the limit $r \rightarrow 0$ in numerical calculations, one has to estimate this dimension from larger distances, i.e. the right-hand side of Eq. (24) becomes a distant dependent function $D_{2}(r, m)$. For low-dimensional attractors and small $r$ there often exists a rather large region in $\log _{2}(r)$ where $D_{2}(r, m)$ is nearly constant. This part is referred to as the scaling region [Grassberger \& Procaccia, 1983]. For larger values of $r$, $D_{2}(r, m)$ is decreasing because of boundary effects. It has been shown, that with growing dimension of the attractor the number of data points needed to reach the scaling region is increasing exponentially [Kantz \& Schreiber, 1997; Raab \& Kurths, 2001]. If the time series is too short, one only gets that part of $D_{2}(r, m)$ where it is decreasing with $\log _{2}(r)$. With LASDID we are able to use this part of $D_{2}(r, m)$, too.

This large-scale dimension density $\rho_{\mathrm{ls}}(r, m)$ is defined by normalizing the dimension density $D_{2}(r, m) / m$ of all coordinates $m$ of the embedded system to the dimension density $D_{2}(r, 1)$ of one coordinate of this system [Raab \& Kurths, 2001]:

$$
\rho_{\mathrm{ls}}(r, m)=\frac{D_{2}(r, m)}{\left(m D_{2}(r, 1)\right)} .
$$

With this normalization we get a plateau for large scales $r$ yielding an estimate of $\rho_{\mathrm{ls}}$ (cf. Fig. 9).

The advantage of LASDID is that it is possible to estimate it from rather short and nonstationary time series [Raab et al., 2006]. So we can cut typical RR-interval time series in $M$ shorter pieces. For each of these short pieces we calculate LASDID via Eq. (25). This leads to a time series consisting of $\rho_{\mathrm{ls}}(t)$. For this time series we calculate the mean value $\mu_{\rho_{\mathrm{ls}}}$ by

$$
\mu_{\rho_{\mathrm{ls}}}=\frac{1}{M} \sum_{i=1}^{M} \rho_{\mathrm{ls}}\left(t_{i}\right),
$$

the standard deviation

$$
\sigma_{\rho_{\mathrm{ls}}}=\sqrt{\frac{1}{M-1} \sum_{i=1}^{M}\left(\rho_{\mathrm{ls}}\left(t_{i}\right)-\mu_{\rho_{\mathrm{ls}}}\right)^{2}}
$$

and the coefficient of variation $c v_{\rho_{\mathrm{ls}}}$ by

$$
c v_{\rho_{\mathrm{ls}}}=\sigma_{\rho_{\mathrm{ls}}} / \mu_{\rho_{\mathrm{ls}}} .
$$

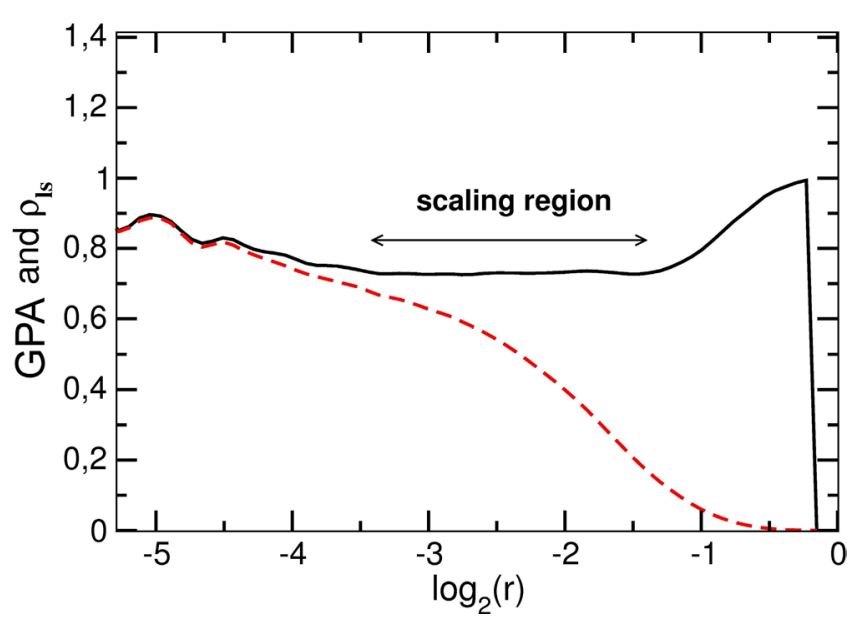

Fig. 9. Comparison of LASDID (solid line) with the Grassberger-Procaccia algorithm (GPA, dashed line) calculated for HRV data. With LASDID we get a plateau for scales between $1 / 2$ and $1 / 10$ of the attractor diameter, corresponding to $\log _{2}(r)=-1$ to -3.4 . For the calculation we used only 2000 RR-intervals, so no scaling region can be found with the Grassberger-Procaccia algorithm. The data was embedded with $\tau=1$ and embedding dimension $m=4$.

For the calculation of LASDID we use an embedding-dimension of $m=4$ and a delay of $\tau=1$. But the results are qualitatively the same with embedding dimensions between $m=4, \ldots, 8$ and delay times between $\tau=1, \ldots, 5$.

\subsubsection{Further nonlinear measures}

In the previous sections several nonlinear measures developed by our group were introduced. This section gives a short survey about further nonlinear methods. One very easy approach is the Poincaré plot, where each value of the time series is plotted as a function of the previous one. We find applications of the Poincaré plot in diverse fields such as astronomy, geophysics, meteorology, mathematical biology and medicine [Ott, 1993]. In medicine it was mainly used in HRV analyses [Woo et al., 1994; Huikuri et al., 1996]. The Poincaré plot is on the one hand a visual technique where the human eye can recognize different patterns, and on the other hand a quantitative one. There are some parameters describing the shape of the plot. A ball-like or stick-like shape in the Poincaré plot has been linked to an adverse prognosis [Woo et al., 1994; Huikuri et al., 1996]. $\mathrm{SD}_{1}$ and $\mathrm{SD}_{2}$ visualized in Fig. 10 are two well-known Poincaré plot measures. $\mathrm{SD}_{1}$ is defined as the standard deviation of the Poincaré plot projection to the negative line of identity, whereas $\mathrm{SD}_{2}$ is the standard deviation 


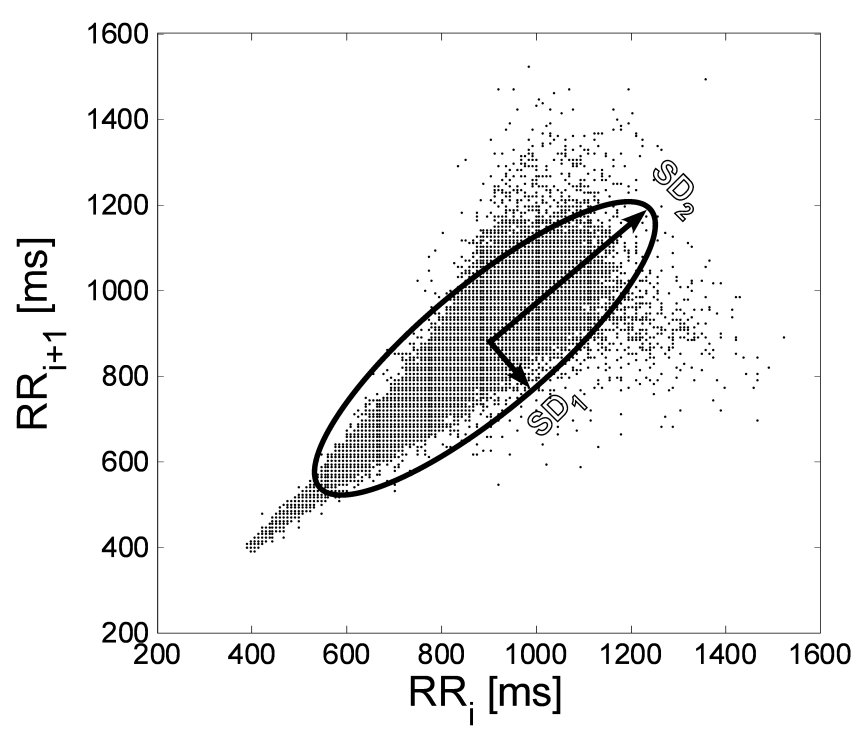

Fig. 10. The Poincaré plot from a 24-hour HRV recording of a healthy 25-year-old man.

of the projection to the line of identity (Fig. 10). $\mathrm{SD}_{1}$ quantifies the short-term variability and $\mathrm{SD}_{2}$ reflects both short-term and long-term variability. The ellipse drawn in Fig. 10 with axes $\left(\mathrm{SD}_{1}, \mathrm{SD}_{2}\right)$ is only a visualization tool for the Poincaré plot volume and its choice is maybe not optimal because the example in Fig. 10 shows a more cudgel-like shape. However, it has not been proven that Poincaré plot based parameters are able to measure nonlinear behavior for HRV data [Brennan et al., 2001].

In physics, entropy is one of the basic measures of the disorder of a system - in information theory, the measure of information capacity. Related to time series analysis, approximate entropy (ApEn), introduced by Pincus [1991], provides a measure of the degree of irregularity or randomness within a series of data. The greater the unpredictability, or complexity, in the time series, the larger the value of ApEn. To calculate the degree of regularity of a time series $x\left(t_{1}\right), \ldots, x\left(t_{N}\right)$, the entropy of recurring patterns is evaluated. Let $C_{i}^{m}(r)$ denote the local recurrence rate of vectors of length $m$ that are close to $\mathbf{x}\left(t_{i}\right)=\left(x\left(t_{i}\right), x\left(t_{i+1}\right), \ldots, x\left(t_{i+m-1}\right)\right)$ for a given distance $r$ :

$$
C_{i}^{m}(r)=\frac{1}{N-m+1} \sum_{j=1}^{N-m+1} \theta\left(r-\left|\mathbf{x}\left(t_{i}\right)-\mathbf{x}\left(t_{j}\right)\right|\right)
$$

where $\theta$ is the Heaviside function and $|\cdot|$ is the maximum norm. Then the averaged $C_{i}^{m}(r)$ denotes the global recurrence rate according to

$$
C^{m}(r)=\frac{1}{N-m+1} \sum_{i=1}^{N-m+1} C_{i}^{m}(r)
$$

The ApEn finally is given by

$$
\begin{aligned}
\operatorname{ApEn}(m, r, N)= & \frac{1}{N-m+1} \sum_{i=1}^{N-m+1} \log C_{i}^{m}(r) \\
& -\frac{1}{N-m+1} \sum_{i=1}^{N-m+1} \log C_{i}^{m+1}(r)
\end{aligned}
$$

and measures the difference between the logarithmic frequencies of similar runs of length $m$ and runs with the length $m+1$. The smaller the ApEn value the higher the regularity in the time series: If the prevalence of recurrent patterns of length $m$ and $m+1$ do not differ significantly, their difference is small. The influence of noise or nonstationarity to ApEn is very high; therefore, it should not be used as a single measure to characterize signals. Moreover, there is an intrinsic bias in the ApEn calculation because the algorithm counts similar sequences to a given sequence, including the sequence itself to avoid a logarithm of 0 in the calculations.

It is important to note here that ApEn is a measure of randomness, i.e. it assigns highest complexity for white-noise series. In this manner ApEn is equivalent to traditional measure of complexity such as the Shannon entropy or the Lempel-Ziv complexity [Ebeling et al., 2002]. The limitations of ApEn led to the development of the sample entropy (SampEn) by Richman and Moorman [2000], where self-matches are excluded. For SampEn the local recurrence rates are defined now as:

$$
C_{i}^{m}(r)=\frac{1}{N-m} \sum_{j \neq i} \theta\left(r-\left|\mathbf{x}\left(t_{i}\right)-\mathbf{x}\left(t_{j}\right)\right|\right)
$$

and the sample entropy SampEn is given by

$$
\operatorname{SampEn}(m, r, N)=\log \frac{\sum_{i=1}^{N-m} C^{m}(r)}{\sum_{i=1}^{N-m} C^{m+1}(r)} .
$$

SampEn has the advantage to be less dependent on time series length and free of the bias caused by self-matching.

Both, ApEn and SampEn, are evaluating differences between recurrent patterns of length $m$ and 
$m+1$, thus they evaluate regularity on one scale only. Therefore, Costa et al. [2002] introduced the multiscale entropy (MSE), where they applied Sam$\mathrm{pEn}$ to the original time series averaged at different scales. The connection of entropy and scale leads to consistent results that both completely ordered and completely random signals are not really complex.

A further nonlinear tool exploits the ubiquitous occurrence of power laws in nature. Power scaling laws have been studied for example in disparate fields such as meteorology, oceanography, medicine, biology, chemistry and ecology [Li].

$$
P(f) \propto f^{-\gamma}
$$

This phenomenon is thought to arise from the system itself; the theory of self-organized criticality was suggested to represent a universal organizing principle [Bak et al., 1987]. The distribution of the power spectral density can be described by the linear inverse power-law relationship of logarithmic power to logarithmic frequency. By plotting a loglog representation of the power spectrum, a straight line is obtained with a slope of approximately $\gamma=1$. In the algorithm, a straight line is fitted using linear regression, and the slope and intercept are obtained. This power law describes a "scale invariant" dynamics of similar patterns at different scales, which is a property of fractals (cf. Benoit B. Mandelbrot [1983]). For medical data, however, sometimes the power law behavior may be over-interpreted and then it is merely an epiphenomenon. Pilgram et al. [Pilgram \& Kaplan, 1999] showed with a simple $1 / f$ heart rate model that the power-law structure occurred due to nonstationarity over fairly short intervals and thus the statistical result of a conceptually very different process.

Introduced by Peng and coworkers [Peng et al., 1994; Peng et al., 1995], detrended fluctuation analysis (DFA) was developed to determine self-similar, i.e. fractal-like correlation properties. This method is rather insensitive to the effects of noise and nonstationarity, and its considerable advantage is that scaling exponents can be calculated even without outlier removal. Variations from external stimuli (such as respiration) are presumed to cause a local effect (on heart rate), whereas variations due to the inherent dynamics of the system are presumed to exhibit long-range correlations. Firstly, the time series $x_{1}, \ldots, x_{N}$ is mean subtracted and integrated: $y(k)=\sum_{i=1}^{k}\left(x_{i}-\bar{x}\right)$, where $\bar{x}$ denotes the mean value of the time series $x_{1}, \ldots, x_{N}$. Then the series $y(k)$ is divided into boxes of equal length $n$. In each box of length $n$, a least squares line, representing the trend in that box, is fitted to the data. The $y$ coordinate of the straight line segments is denoted by $y_{n}(k)$. Next, $y(k)$ is detrended by subtracting the local trend, $y_{n}(k)$, in each box. The root-meansquare fluctuation of this integrated and detrended time series is calculated by

$$
F(n)=\sqrt{\frac{1}{N} \sum_{k=1}^{N}\left(y(k)-y_{n}(k)\right)^{2}}
$$

This calculation is repeated over all box sizes to get a functional relationship between $F(n)$ and the box size $n$. Typically, $F(n)$ increases with the box size $n$. A linear relationship on a $\log -\log$ plot indicates the presence of power law scaling. The scaling exponent is the slope of the line relating $\log F(n)$ to $\log n$. For real life applications however, the linear relationship between $\log F(n)$ and $\log n$ often has been noted to be distinct for small $n(n \leq 11)$ and large $n(n>11)$, yielding two lines with two slopes, labeled the scaling exponents $\alpha_{1}$ and $\alpha_{2}$, respectively [Makikallio et al., 1998]. Theoretically, the scaling exponent(s) will vary from 0.5 (uncorrelated randomness) to 1.5 (random walk process, Brownian noise), but physiological signals usually have scaling exponents around 1.

The DFA is a monofractal technique, it can measure only one exponent characterizing a given signal. Monofractals are homogeneous in the sense that they have the same scaling law throughout the entire signal. Multifractal techniques provide a spectrum of multiple exponents. Many physiological time series are inhomogeneous due to intrinsic and extrinsic influences, suggesting that different parts of the signals have different scaling properties. For example, Stanley introduced 1999 [Stanley et al., 1999] a multifractal analysis technique similar to DFA, which is capable of distinguishing the heart rate dynamics of patients with congestive heart failure from healthy control individuals. A more detailed description of multifractality in biological signals can be found in the papers by Ivanov [Ivanov et al., 1999; Ivanov et al., 2001]. For medical data analysis however, it remains unclear whether these techniques will lead to clinically significant improvements.

\subsection{Baroreceptor sensitivity}

The analysis of the spontaneous baroreflex sensitivity (BRS) is very important for cardiac risk stratification of different cardiovascular diseases [Bristow 
et al., 1969; Eckberg et al., 1971; Barron \& Lesh, 1996; La Rovere et al., 2001]. The BRS is defined as the instinctive change of the heart frequency (beatto-beat interval) related to increasing or decreasing values in systolic blood pressure and is expressed in $\mathrm{ms} / \mathrm{mmHg}$. There is evidence that a decreased BRS may carry an adverse prognosis in cardiac patients [Osterziel et al., 1995; Mortara et al., 1997; La Rovere et al., 1998]. For several years, the BRS was determined pharmacologically (phenylephrine, nitro-prusside) [McCall \& Humphrey, 1983; Vanoli \& Adamson, 1994] or mechanically [Cohen et al., 1981; Mancia et al., 1984; Takahashi et al., 1999] until in the 1980s innovative methods for BRS estimation were developed which are based on spontaneous heart rate and blood pressure fluctuations [Di Rienzo et al., 1985; Parati et al., 1988]. These methods evaluate arterial baroreflex function in the absence of external stimulations on the cardiovascular system, therefore defined as spontaneous. These spontaneous techniques nowadays are state of the art in research, but not in clinical practice. The next paragraph provides a brief survey of these approaches.

Di Rienzo and Parati introduced in the 1980s the sequence method [Di Rienzo et al., 1985; Parati et al., 1988] which was the first not requiring drug-injections. This time-domain method scans the beat-to-beat systolic pressure series to identify sequences with monotonic BP increases (or decreases) and synchronous BBI increases (or decreases). The slope of the regression line between $\mathrm{BBI}$ and systolic blood pressure (SBP) values within the sequence is taken as an estimate of BRS. Robbe et al. introduced a transfer function based method [Robbe et al., 1987] for estimating the BRS. This method assumes that the BBI is the output of a linear system in which systolic pressure is the input. Another frequency domain method was introduced by Pagani et al. [1988] and uses the fact that spontaneous fluctuations of SBP and BBI are linearly correlated at the respiratory $\mathrm{HF}$ as well as at the LF frequency band. The alpha method assumes that this correlation is due to baroreflex cardiovascular control. If the coherence between BBI and SBP exceeds a certain value, an estimate of BRS is calculated by the root-squared ratio $\left(\alpha_{\mathrm{LF}}\right.$ resp. $\left.\alpha_{\mathrm{HF}}\right)$. An alternative way to quantify BRS was introduced by Ducher et al. [1995], where the relation between SBP values and BBI is assessed statistically. The statistical level of coupling is quantified by the Z-index, which is a function of SBP and BBI. BRS finally is derived from the shape of the Z-surface on the SBP-BBI plane. Further approaches for estimating BRS are based on mathematical modeling of cardiovascular circulation. The model coefficients are fitted from the experimental data and the BRS is derived from the model parameters. Some examples for these approaches are dynamic adjustment models [Baselli et al., 1988], autoregressive-moving average models [Patton et al., 1996], exogenous models with autoregressive input [Porta et al., 2000], and bivariate autoregressive models [Nollo et al., 2001; Nollo et al., 2005]. Recently, Westerhof et al. [2004] introduced the xBRS method, which is a direct extension of the sequence method. Again BRS is obtained as the slope of the regression line between SBP and BBI. However, the sequences do not need to be synchronous. Corresponding sequences are found by a significant maximum of the crosscorrelation function between SBP and BBI in a 10 -s window. In this way different response times of heart rate to SBP changes can be considered.

We have developed the dual sequence method (DSM) [Malberg et al., 1999; Malberg et al., 2002] for the advanced spontaneous baroreflex sensitivity estimation. This method considers not only bradycardic (blood pressure increase causes RRinterval increase) and tachycardic (blood pressure decrease causes RR-interval decrease) blood pressure fluctuations as introduced in the sequence method [Di Rienzo et al., 1985; Parati et al., 1988] [see Fig. 11(a)], but also defines slope sectors quantifying the BRS-slope distribution [see Fig. 11(b)]. Former studies showed that the heart rate does not respond simultaneously to the blood pressure fluctuation [Mancia et al., 1984; Mancia et al., 1985]. Therefore, DSM quantifies synchronous as well as postponed heart rate response on the same BP fluctuation [see Fig. 11 (c)].

In summary, the following parameter blocks are calculated by DSM:

(i) the total number of slopes in the different sectors within the time series,

(ii) the percentage of the slopes to the total number of slopes in the different sectors,

(iii) the numbers of bradycardic and tachycardic slopes,

(iv) the shift operation from the first to the third heart beat triple and

(v) the average slopes of all fluctuations. 


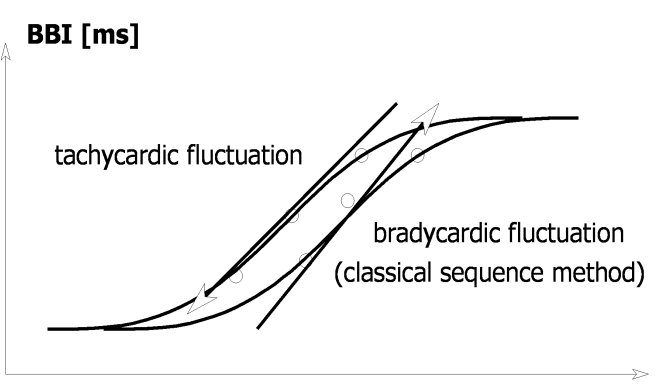

syst. BP [mmHg]

(a)

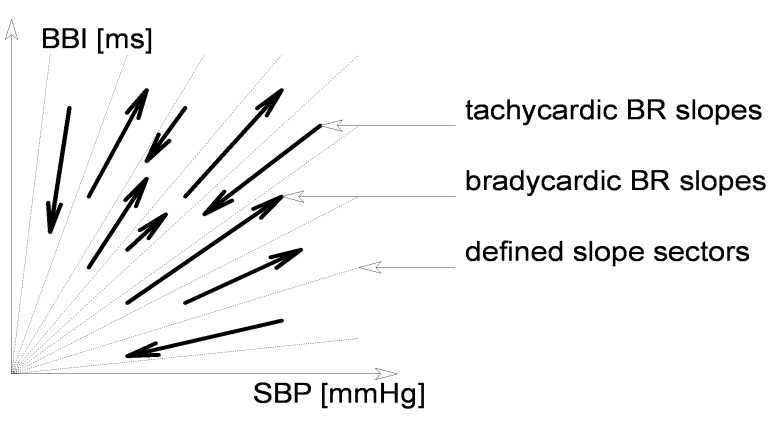

(b)

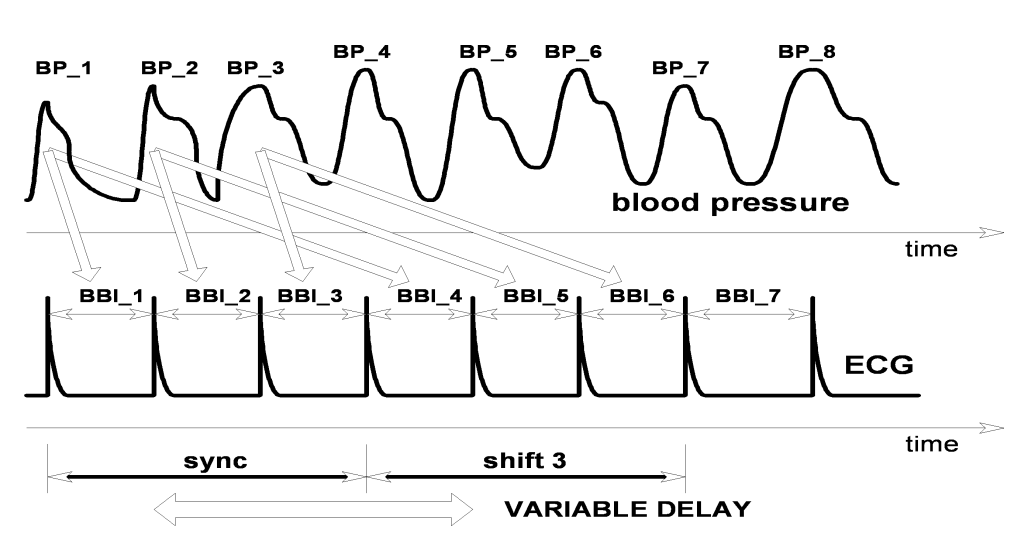

(c)

Fig. 11. The dual sequence method for spontaneous BRS estimation includes in addition to the bradycardic and tachycardic blood pressure fluctuations (classical sequence method, (a)) also the slope sector distribution (b). Moreover, not only simultaneous (sync) but also delayed responses of heart rate to blood pressure increases (shift 3, variable delay) are analyzed.

The average BRS slope is defined as the beat-tobeat interval difference related to SBP changes, estimated by linear regression

$$
\mathrm{BRS}=\frac{\mathrm{BBI}}{\mathrm{SBP}}
$$

and expressed in $\mathrm{ms} / \mathrm{mmHg}$.

The parameters Pbrady and Ptachy characterize the incidence of increasing and decreasing SBP tripels with regard to the total number of SBP values. Consequently, these parameters estimate the basic cause of BRS activity. An reduced number of ramps in SBP leads unavoidably to a reduced number of $\mathrm{HR}$ responses. The parameters are defined as:

$$
\begin{aligned}
& \text { Pbrady }=\left(\frac{\text { No. of increasing SBP ramps }}{\text { total no. of SBP values }}\right) \cdot 100 \% \\
& \text { Ptachy }=\left(\frac{\text { No. of decreasing SBP ramps }}{\text { total no. of SBP values }}\right) \cdot 100 \% .
\end{aligned}
$$

The percentage of adequate $\mathrm{HR}$ responses (BRS events) to the numbers of SBP ramps is described by the parameter Activation. It is definded as:

$$
\text { Activation }=\left(\frac{\text { No. of BRS events }}{\text { No. of SBP ramps }}\right) \cdot 100 \% \text {. }
$$

In contrast to classical BRS methods, the DSM defines slope sectors allowing to quantify the BRS-slope distribution. Sectors with a range of $5 \mathrm{~ms} / \mathrm{mmHg}$ have been proven to act as a suitable partition. The slope sectors are defined as:

Slope Sector $[\mathrm{ms} / \mathrm{mmHg}]$ :

'0 - 5' (very low BRS-slopes, most noise)

Slope Sector $[\mathrm{ms} / \mathrm{mmHg}]$ :

$'<5^{\prime},{ }^{\prime} 5-10^{\prime}, \ldots,,^{\prime} 25-30^{\prime},{ }^{\prime}<30^{\prime}$

(expected physiological BRS)

Then, the percentages of BRS events in different slope sectors to the total BRS number can be estimated. Moreover, these values are normalized to the mean heart rate. For detailed definitions of the DSM parameters we refer to the original contribution [Malberg et al., 2002]. These parameters are calculated for bradycardic as well as for tachycardic 
fluctuations, which are synchronous or delayed, to analyze a possibly delayed response of the heart rate to the same blood pressure oscillation. Using this DSM method, sequences of length three are quantified; longer sequences turned out not to be useful for spontaneous BRS estimation because of their low occurrence.

\subsection{Heart rate turbulence}

Some years ago, the method of heart rate turbulence (HRT) was introduced [Schmidt et al., 1999b], analyzing only ventricular premature complexes (VPC) and the behavior of the surrounding normal beats. The HRT method was shown to represent a significant extension of variability analysis in myocardial infarction patients [Schmidt et al., 1999b]. It was demonstrated that the counter-regulation of the NN-intervals (normal-beat to normal-beat, NNI) directly after VPC yields further information for the prognosis following myocardial infarction. After a VPC, the heart rate increases for 1 or 2 beats, then it decreases (cf. Fig 12).

HRT quantifies these heart rate changes by two parameters, the turbulence onset (TO) and the turbulence slope (TS). TO is defined as the percentage difference between two post- and two pre-arrhythmic beats

$$
\mathrm{TO}=\frac{\left(\mathrm{NNI}_{2}+\mathrm{NNI}_{3}\right)-\left(\mathrm{NNI}_{-2}+\mathrm{NNI}_{-1}\right)}{\left(\mathrm{NNI}_{-2}+\mathrm{NNI}_{-1}\right)} \cdot 100 \%,
$$

where the numbers denote the beat number, with the VPC being beat 0 . In other words, TO is the amount of heart rate increase following a VPC. TS is defined as the maximal slope of five successive normal beats following the compensatory pause after the VPC. In [Schmidt et al., 1999b] it was suggested to calculate TS from 16 post-VPC beats. In short, TS is the amount of heart rate deceleration after the VPC caused heart rate acceleration. For long-term ECGs the TO and TS values for each VPC of one patient are averaged. Patients after myocardial infarction with a good prognosis were characterized by the following response: The heart rate immediately after the VPC increases compared to the heart rate before the VPC, thus $T O<0$. Moreover, there is a relatively strong heart rate recovery after the $\mathrm{VPC}$ induced heart rate increase, thus TS $>2.5 \mathrm{~ms} /$ beat [Schmidt et al., 1999b]. Patient who died in the follow up of this

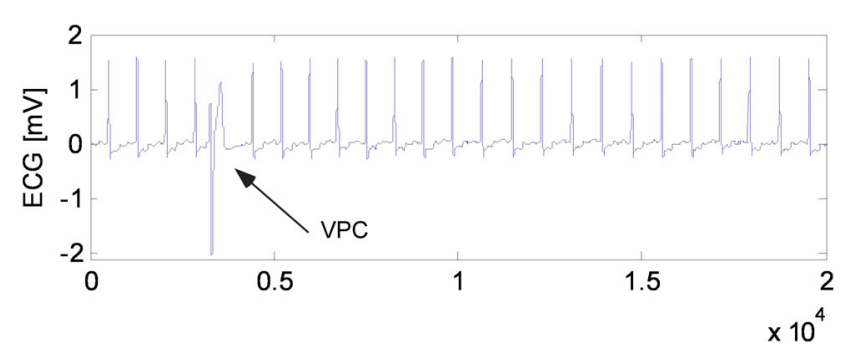

(a)

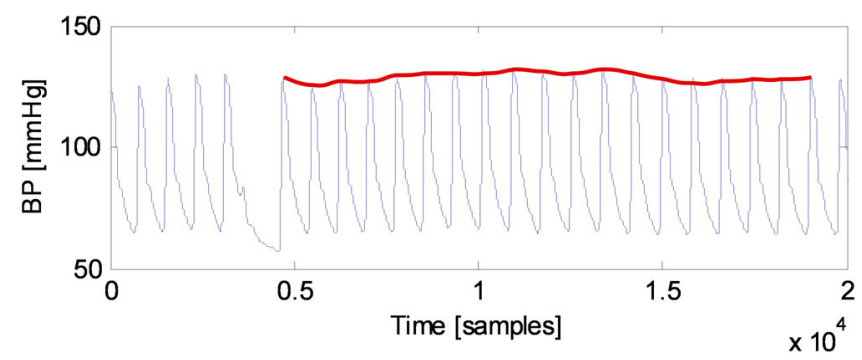

(b)

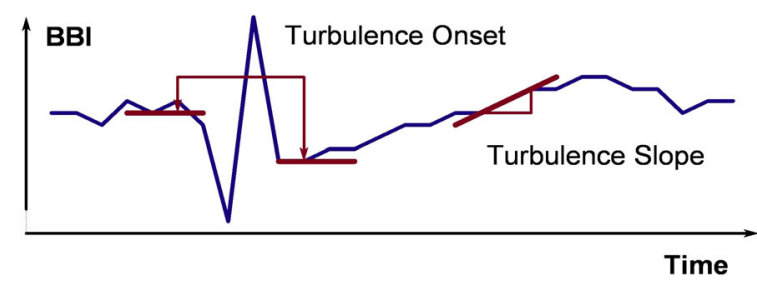

(c)

Fig. 12. Heart rate turbulence phenomenon: The time series in (a) shows a ventricular premature complex (VPC) in the ECG. Apparently, the dynamics after the VPC is not influenced. Looking however at the blood pressure oscillations (b), one can recognize the following behavior: the first blood pressure value after the VPC has approximately the same amplitude as the last normal beat, afterwards we see a short blood pressure decrease followed by an increase up to ten values after the VPC (red line). This phenomenon is better visible when plotting the beat-to-beat intervals from (a) versus time (c). This tachogram is characterized by a decrease of BBI after VPC (turbulence onset) and a pronounced BBI-wave (turbulence slope).

study very often showed $\mathrm{TO}>0$, i.e. a heart rate decrease immediately after the VPC, and no turbulence slope.

HRT analysis is carried out preferably with 24-hour ECGs. However, shorter measurement durations are not excluded by the method [Schmidt et al., 1999b]. To analyze HRT also for short term recordings, where only beat-to-beat intervals were available, the classical $\mathrm{NN}$-interval trigger, 
suggested by Schmidt et al. [1999b], was extended for the detection of VPCs. VPC detection by this trigger is based on the fact that the premature beat has to be smaller than $80 \%$ and the following post-extrasystolic pause has to exceed $120 \%$ of the last five normal beats (under the condition: NN-intervals $>200 \mathrm{~ms}$, NN-intervals $<2000 \mathrm{~ms}$ ). Another pre-requisite is that the five pre- and 18 post-extrasystolic heart beats are normal beats (post-extrasystolic: NN-intervals after the compensatory pause). When using this trigger, however, arrhythmias, artifacts, and extraction errors of the noninvasive blood pressure measurement device were found just in this range prior to and after the VPC. For this reason, an automatic method was introduced, which facilitates VPC evaluation [Malberg et al., 2003, 2004].

\subsection{Nonlinear model based data analysis}

One aim of this tutorial is to take a qualitatively new step: the combination of data analysis and modeling. Our nonlinear-dynamics approach of model-based data analysis is based on the concept of maximal correlation, which is very powerful to estimate dependencies of one or more especially nonlinear related variables [Renyi, 1970]. This approach provides a nonparametric procedure to detect and determine nonlinear relationships in multivariate data sets. For simplification reasons we start with the bivariate case. Let $X$ and $Y$ denote two zero-mean data sets and

$$
\rho(X, Y)=\frac{E[X Y]}{\sqrt{E\left[X^{2}\right] E\left[Y^{2}\right]}},
$$

their linear correlation coefficient, where $E[\cdot]$ is the expectation value. The basic idea of this approach is to find such transformations $\theta(Y)$ and $\phi(X)$ that the absolute value of the correlation coefficient between the transformed variables is maximized. This leads to the maximal correlation [Hirschfeld, 1935; Gebelein, 1941; Renyi, 1970].

$$
\Psi(X, Y):=\sup _{\theta, \phi}|\rho(\theta(X), \phi(Y))|
$$

The functions $\theta(Y)$ and $\phi(X)$ for which the supremum is attained are called optimal transformations. This concept generalizes the linear correlation, where the linear correlation coefficient $\rho(X, Y)$ quantifies only linear dependencies

$$
Y=a X+\eta
$$

while $\Psi(X, Y)$ quantifies nonlinear dependencies of the form

$$
\Psi(Y)=\phi(X)+\eta .
$$

Especially, if there is complete statistical dependence [Renyi, 1970], i.e., $Y$ is a function of $X$ or vice versa, the maximal correlation attains unity. This is also true for the Eq. (44) with $\eta=0$. In Fig. 13 an example of estimating functional relationships is given. There, a cubic relationship with multiplicative and additive noise is shown. Using

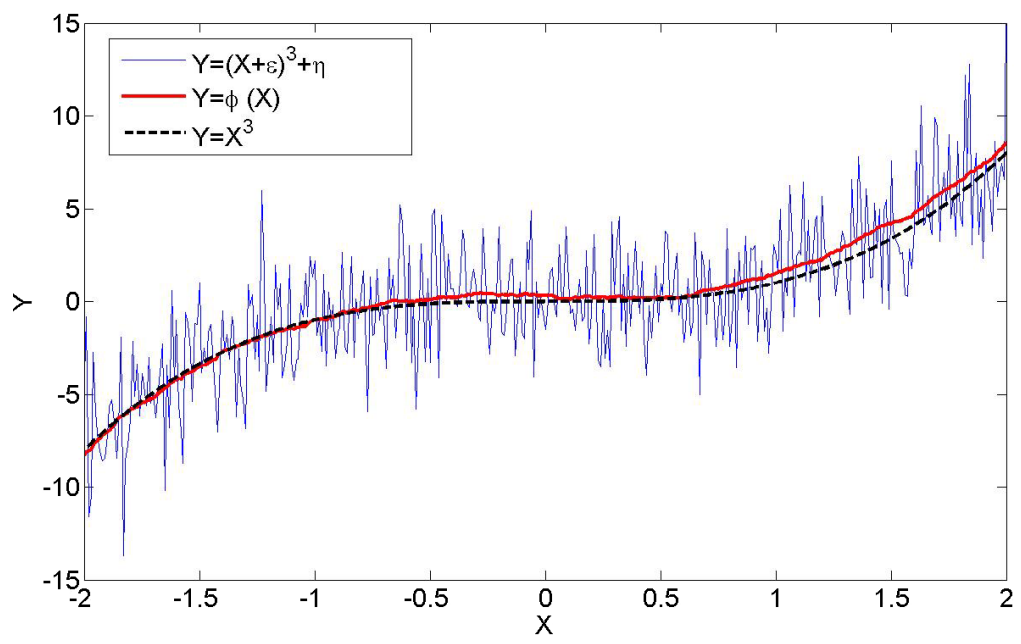

Fig. 13. A realization of a cubic function with multiplicative and additive noise is depicted $\left(Y=(X+\varepsilon)^{3}+\eta, \varepsilon \sim N(0 ; 0.2)\right.$, $\eta \sim N(0 ; 2))$. The linear correlation amounts to $r=0.83$. Using the nonlinear regression approach we get a higher dependence with a maximal correlation of $\Psi=0.9$ ( $\phi$ - optimal transformation). The function $Y=X^{3}$ is plotted for comparability reasons. 
the concept of maximal correlation the nonlinear relationship between $X$ and $Y$ can be revealed and quantified.

In this tutorial we are mainly interested in the estimation of the optimal transformations for the multivariate regression problem

$$
\theta(Y)=\sum_{i=1}^{k} \phi_{i}\left(X_{i}\right)+\eta
$$

This is an additive model for the (not necessarily independent) input variables $Y, X_{1}, \ldots, X_{k}$. The regression functions involved can be estimated as the optimal transformations for the multivariate problem analogous to Eq. (42). To estimate them nonparametrically, we use the Alternating Conditional Expectation (ACE) algorithm [Breiman \& Friedman, 1985; Hastie \& Tibshirani, 1990]. This iterative procedure is nonparametric because the optimal transformations are estimated by local smoothing of the data using kernel estimators. We use a modified algorithm in which the data are rank-ordered before the optimal transformations are estimated. This makes the result less sensitive to the data distribution. The maximal correlation and optimal transformation approach have recently been applied to nonlinear dynamical systems especially to model river flow data [Chen \& Tsay, 1993], to identify delay in lasers [Voss \& Kurths, 1997] and partial differential equations in fluid dynamics [Voss et al., 1999], to predict thermal displacements in modular tool systems [Wessel et al., 2004a; Wessel et al., 2004b] and to medical data analyses [Wessel et al., 2000b; Wessel et al., 2006]. A more general review on nonlinear system identification is given in [Voss et al., 2004].

\section{Clinical Applications}

After having briefly introduced the methods of cardiological biosignal processing this section of the tutorial will give an overview of selected clinical applications.

\subsection{Risk stratification for sudden cardiac death based on HRV data}

More than ten years ago, we started applying the concept of complexity measures to determine the risk of sudden cardiac death based on HRV data [Kurths et al., 1995]. Therefore, we analyzed the 30 minutes tachograms from 43 subjects, which were recorded under rest conditions. This pilot study included 21 healthy volunteers, 9 patients after myocardial infarction with low electrical risk and 13 patients after myocardial infarction with documented life-threatening arrhythmias (sustained ventricular tachycardia). We showed that by using classical methods only, especially parameters from the time domain, the individual risk cannot be defined precisely enough. We found some indication that two kinds of complexity measures are particularly promising: symbolic dynamics and renormalized entropy. In that paper we were the first to show that one parameter is insufficient to satisfactorily define the individual risk. A combination of several quantities which refer to different aspects, such as structural or dynamical aspects, seemed to be the most promising way.

Later, a second study introduced new methods of nonlinear dynamics and compared these with traditional methods of HRV and high resolution ECG (HRECG) analysis in order to improve the reliability of high risk stratification [Voss et al., 1996b]. In this study, simultaneous 30 minutes high resolution ECGs and long term ECGs were recorded from 26 cardiac patients after myocardial infarction. Again, they were divided into two groups depending upon the electrical risk, a low risk group (group $2, n=10$ ) and a high risk group (group $3, n=16$ ). The control group consisted of 35 healthy persons (group 1). From these electrocardiograms we extracted standard measures in time and frequency domain as well as measures from the new nonlinear methods of symbolic dynamics and renormalized entropy. Applying discriminant function techniques to HRV analysis, the parameters of nonlinear dynamics led to an acceptable differentiation between healthy persons and high risk patients of $96 \%$. The time domain and frequency domain parameters were successful in less than $90 \%$. The combination of parameters from all domains and a stepwise discriminant function separated these groups completely (100\%). Use of this discriminant function classified three patients with apparently low (no) risk into the same cluster as high risk patients. The combination of HRECG and HRV analysis showed the same individual clustering but in addition increased the positive value of separation. In conclusion, the methods of nonlinear dynamics describe complex rhythm fluctuations and separate pattern of nonlinear behavior in the heart rate time series more successful than classical methods of time and frequency domains. This leads to an improved discrimination between a normal (healthy 
persons) and an abnormal (high risk patients) type of heart beat generation. Some patients with an unknown risk exhibit similar patterns as high risk patients and this suggests a hidden high risk. The methods of symbolic dynamics and renormalized entropy were particularly useful measures for classifying the dynamics of HRV.

The objective of a subsequent blinded study was to prove that this multiparametric HRV analysis improves the result of risk stratification in patients after myocardial infarction [Voss et al., 1998]. Standard time domain, frequency domain and nonlinear dynamics measures of HRV assessment were applied to 572 survivors of acute myocardial infarction. Three parameter sets each consisting of four parameters were applied and compared with the standard measurement of global heart rate variability HRVi. Discriminant analysis technique and $t$-test were performed to separate the high risk groups from the survivors. The predictive value of this approach was evaluated with receiver operator (ROC) and positive predictive accuracy (PPA) curves. The discriminant analysis showed a separation of patients suffered by all cause mortality in $80 \%$ (best single parameter $74 \%$ ) and sudden arrhythmic death in $86 \%$ (73\%). All parameters of set 1 (the Shannon entropy of the histogram, the normalized very low frequency, the symbolic dynamics parameter WPSUM02, the mean beatto-beat intervals from the most stationary stage) showed a high significant difference $(p<0.001)$ between survivors and nonsurvivors based on a twotailed $t$-test. The specificity level of the multivariate parameter sets was at the $70 \%$ sensitivity level (ROC) about 85-90\%, whereas HRVi showed maximum levels of $70 \%$ (Fig. 14). The PPA in the all cause mortality group was at the $70 \%$ sensitivity level twice as high as the univariate HRV measure and increased to more than fourfold as high within the VT/VF group. For this population we could conclude, that the combination of four parameters from all domains especially from nonlinear dynamics seems to be a better predictor of high arrhythmia risk than the standard measurement of global heart rate variability.

As shown in previous studies, standard time and frequency parameters of HRV describe only linear and periodic behavior, while more complex relations cannot be recognized. On the other hand, the "renormalized entropy" was proven to be capable of assessing more complex properties. However, a previously introduced method [Kurths et al., 1995;

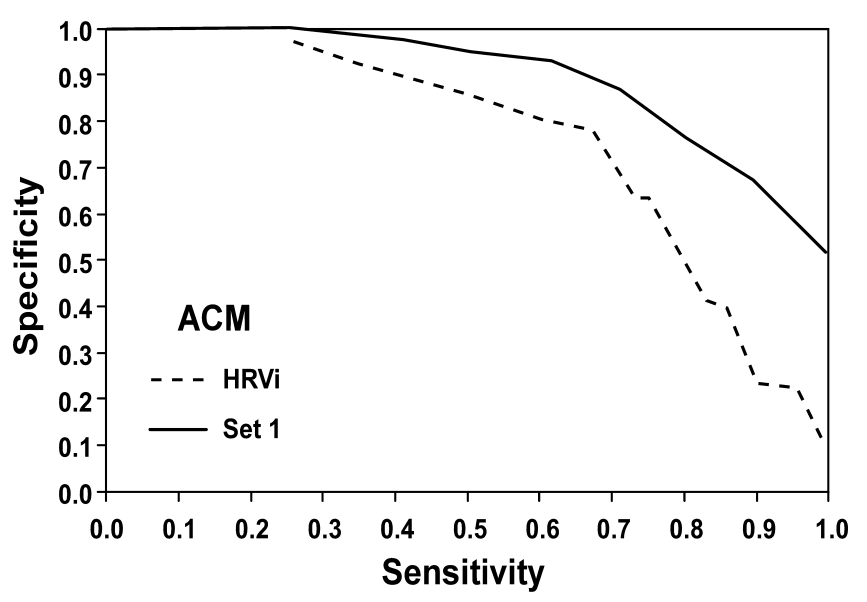

Fig. 14. Receiver operator curves for the all cause mortality (ACM) group and parameter HRVi respectively parameter set 1 .

Voss et al., 1996b] using renormalized entropy suffered from a potential lack of reproducibility and time instability. To overcome these limitations, a new concept of the method, $\mathrm{RE}_{\mathrm{AR}}$, was developed (cf. Sec. 2.3.2) based on a nonlinear renormalization of autoregressive spectral distributions [Wessel et al., 2000a]. To test the hypothesis that renormalized entropy may improve the result of high risk stratification after myocardial infarction, it was applied to a clinical pilot study (41 subjects) and to prospective data of the St. George's Hospital post-infarction data base (572 patients). The study showed that the new $\mathrm{RE}_{\mathrm{AR}}$ method is more reproducible and more stable in time than a previously introduced method $(p<0.001)$. Moreover, the results of the study confirmed the hypothesis that on average, the survivors have negative values of $\mathrm{RE}_{\mathrm{AR}}(-0.11 \pm 0.18)$ whereas the nonsurvivors have positive values $(0.03 \pm 0.22, p<0.01)$. Further, the study showed that the combination of HRV triangular index and $\mathrm{RE}_{\mathrm{AR}}$ leads to a better prediction of sudden arrhythmic death than standard measurements of HRV. In summary, the $\mathrm{RE}_{\mathrm{AR}}$ method is an independent measure in HRV analysis which might be suitable for risk stratification in patients after myocardial infarction.

\subsection{Forecasting of life-threatening cardiac arrhythmias}

Ventricular tachycardia or fibrillation (VT) are the main factors triggering sudden cardiac death (Fig. 15). The objective of the pilot study in 1998 [Wessel et al., 2000c] was to find early signs of sustained VT in patients with an implanted 


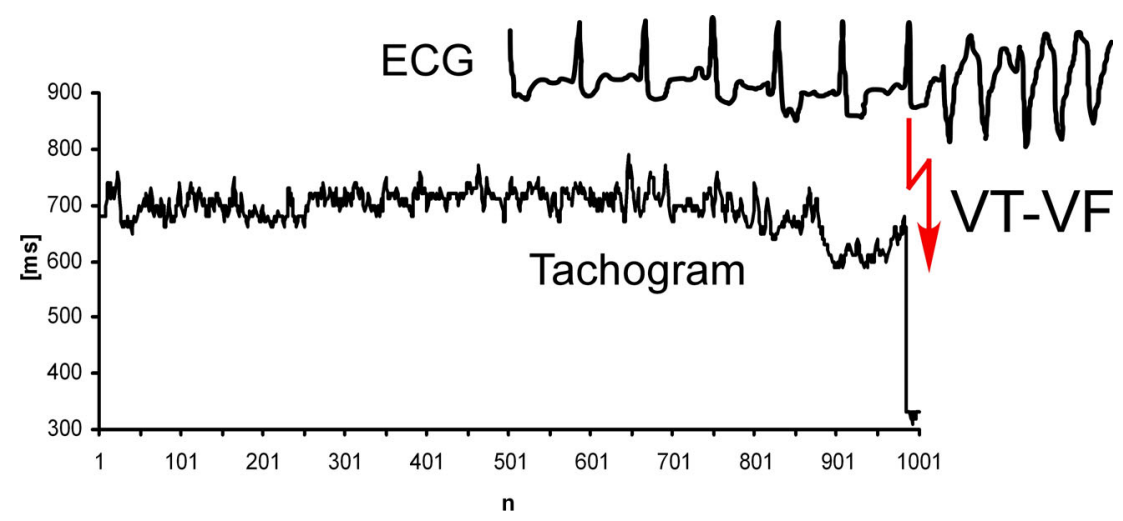

Fig. 15. 1000 beat-to-beat intervals (tachogram) before the onset of ventricular tachycardia or fibrillation (VT). In the ECG this life-threatening arrhythmia is characterized by abnormal-looking QRS complexes with a rate greater than 100 beats/minute.

cardioverter-defibrillator (ICD). These devices are able to safeguard patients by returning their hearts to a normal rhythm via strong defibrillatory shocks; additionally, they store the 1,000 beat-to-beat intervals immediately before the onset of a lifethreatening arrhythmia. We studied these 1,000 beat-to-beat intervals of 17 chronic heart failure ICD patients before the onset of a life-threatening arrhythmia and at a control time, i.e. without VT event. To characterize these rather short data sets, we calculated heart rate variability parameters from time and frequency domain, from symbolic dynamics as well as the finite-time growth rates. We found that neither the time nor the frequency domain parameters show significant differences between the VT and the control time series. However, two parameters from symbolic dynamics as well as the finite-time growth rates discriminate significantly both groups. We concluded that these findings could be of importance in algorithms for next generation ICDs to improve the diagnostics and therapy of VT. We therefore initiated at that time a multicenter study to collect not only 1,000 but 9,000 beat-to-beat intervals before the onset of VT (MARITA-study: Multivariate Analysis of RRIntervals before the onset of TAchyarrhythmias).

In a further study, we tested whether or not HRV changes can serve as early signs of VT and predict slow and fast VT in patients with an ICD [Meyerfeldt et al., 2002]. Therefore, we studied the ICD stored 1,000 beat-to-beat intervals before the onset of VT (131 episodes) and during a control time without VT (74 series) in 63 chronic heart failure ICD patients. Standard HRV parameters as well as two nonlinear parameters, namely "POLVAR10" from symbolic dynamics and the finite time growth rates "Fitgra9" were calculated. Comparing the control and the VT series, no linear HRV parameter showed a significant difference. The nonlinear parameters detected a significant increase in short phases with low variability before the onset of VT (for time series with less than $10 \%$ ectopy, $p<0.05)$. Subdividing VT into fast (cycle length $\leq 270 \mathrm{~ms}$ ) and slow $(>270 \mathrm{~ms})$ events we found that the onset of slow VT was characterized by a significant increase in heart rate, whereas fast VT was triggered during decreased heart rates, compared to the control series. In summary, our analysis indicates that one may develop new automatic ICD algorithms based on nonlinear dynamic HRV parameters to predict VT before it starts.

In a new analysis of the pilot study from 1998 we proposed measures of complexity based on vertical structures in recurrence plots [Marwan et al., 2002]. These measures make the investigation of intermittency of processes possible, even if they are only represented by short and nonstationary data series. For the logistic map these measures (cf. Sec. 2.3.4) enabled us not only to detect transitions between chaotic and periodic states, but also to identify laminar states, i.e. chaos-chaos transitions. The traditional recurrence quantification analysis failed to detect the latter transitions. Applying our new measures to the HRV data of the pilot study, we were able to detect and quantify the laminar phases before the occurrence of life-threatening cardiac arrhythmia. The differences between VT and the control series were more significant for RQA parameters than for measures used in [Wessel et al., 2000c]. This study also demonstrated the importance of developing sophisticated 


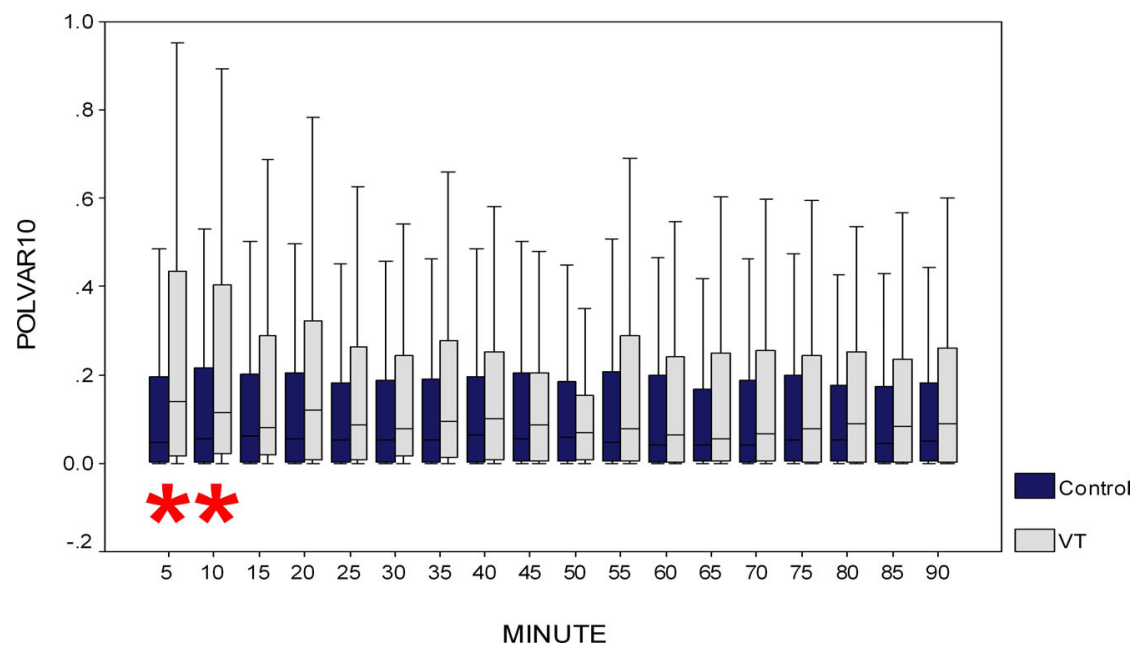

Fig. 16. The probability of intermittently low variability POLVAR10 up to 90 minutes before the onset of VT $(p<0.001$ only 5 and 10 minutes before VT).

nonlinear dynamical tools for the analysis (not only) of medical data.

In 2004 finally, we presented the first results of the MARITA study [Schirdewan et al., 2004]. In this study, one hundred eighty-six patients (153 men, mean age $61.0 \pm 10.4$ ) with an implanted ICD (Biotronik, 9000 RR-intervals capacity) for recurrent VT provided 257 heart rate recordings before a VT (94 patients) and 645 series during control conditions: recordings at follow-up date without VT. From these time series we analyzed 107 VT and 422 control recordings which were in sinus rhythm, more than one hour long and had less than $10 \%$ ventricular ectopy. For HRV analysis standard time- and frequency parameters as well as nonlinear dynamical measures were calculated. To determine the time of sympathetic activation, we calculated all HRV parameter for successive 5 minutes intervals up to 90 minutes before the onset of VT. In this data base, the mean RR-interval for VT was significantly lower than for the controls $(752.4 \pm 13.9$ vs. $819.1 \pm 8.1, p<0.0001)$, whereas the number of ventricular premature beats was higher $(230.5 \pm 19.7$ vs. $147.7 \pm 10.5, p<0.00001)$. Heart rate and ventricular ectopy rate significantly increase already 90 minutes before VTA onset compared with control conditions suggesting a state of sympathetic excitation. Mean heart rate and the number of VPCs were statistically significant for all comparisons. In addition the symbolic dynamics parameter, POLVAR10 detected a further sympathetic excitation $10 \mathrm{~min}$ utes before the onset of VT, whereas all other parameters failed to detect these changes. Stepwise discriminant function analysis gave a classification rate between VT and control series of $77.1 \%$ with a positive predictive accuracy of $51.4 \%$ and a negative predictive accuracy of $88.3 \%$. These first results of the MARITA study suggest, that monitoring of heart rate dynamics in ICD patients can predict VT events with a positive accuracy for VT of about $50 \%$. Further analyses of this study led us to the idea of a circadian ICD, where the positive predictive accuracy for VT could drastically increase (International patent registration PCT/DE2005/ 002040).

\subsection{Cardiovascular variability in hypertensive pregnancy disorders}

When using HRV-, BPV- or BRS-methods as markers for hypertensive pregnancy disorders, it is first necessary to describe these parameters in normal pregnancy [Voss et al., 2000]. To accommodate the complexity of autonomic cardiovascular control, we added parameters of nonlinear dynamics to conventional linear methods of time and frequency domains. The BP of 27 women with normal pregnancy and 14 nonpregnant women were monitored at a high resolution $(200 \mathrm{~Hz}$ sampling frequency) using a Portapres for $30 \mathrm{~min}$. The pregnant women were divided into groups of "32 or less" and "greater than 32" weeks of gestation. Pregnant and nonpregnant women were classified into subclasses of maternal age of less than 28 years resp. older. Except for two single parameter domains, we found no significant differences in HRV and BPV for pregnant women with different 
gestational age or different maternal age. Moreover, no significant differences in spontaneous BRS could be found between pregnant women regardless of either their age or gestational age. In contrast, all measures of nonlinear dynamics of HRV as well as all parameter domains of spontaneous BRS showed significant changes between pregnant and nonpregnant women, whereas BPV did not differ between those groups. This complex assessment of autonomic cardiovascular regulation has shown that the parameters tested are stable in the second half of normal pregnancy, and might have the potential to be excellent indicators of pathophysiologic conditions.

After having demonstrated the potential of our methods, we investigated the pathophysiology of hypertensive pregnancy disorders, which are a leading cause of perinatal and maternal morbidity and mortality. The aim of this study [Faber et al., 2004] was to evaluate, whether HRV, BPV, and BRS differ between distinct hypertensive pregnancy disorders. Continuous heart rate and blood pressure recordings were performed in 80 healthy pregnant women as controls (CON), 19 with chronic hypertension $(\mathrm{CH}), 18$ with pregnancy-induced hypertension $(\mathrm{PIH})$, and 44 with preeclampsia $(\mathrm{PE})$. The data were assessed by time and frequency domain analysis, nonlinear dynamics and BRS. BPV is markedly altered in all three groups with hypertensive disorders compared to healthy pregnancies, whereby changes were most pronounced in PE patients. Interestingly, this increase in $\mathrm{PE}$ patients did not lead to elevated spontaneous baroreflex events, while BPV changes in both other hypertensive groups were paralleled by alterations in baroreflex parameters. The HRV was unaltered in $\mathrm{CH}$ and $\mathrm{PE}$ but significantly impaired in PIH. We conclude that parameters of HRV, BPV, and BRS differ between various hypertensive pregnancy disorders. Thus, distinct clinical manifestations of hypertension in pregnancy have different pathophysiological, regulatory and compensatory mechanisms.

Nowadays, the estimated prevalence of chronic hypertension in pregnancy is $3 \%$ - more than 100,000 of the pregnant women in the United States are affected per year. The aim of a further study [Walther et al., 2005] was to investigate the adaptation of autonomic control during pregnancy based on HRV analysis and to prove whether chronic hypertension during pregnancy has an impact on this adaptation. Thirty-five healthy pregnant women (CON, age: median 28, range [24-30] years) and 16 chronic hypertensive women $(\mathrm{CH}$, age: 30 [25-33] years) were recruited for this longitudinal study. Beginning at the 20th week of pregnancy, the women were monitored every fourth week until delivery. For the analysis of HRV, Portapres signals $(200 \mathrm{~Hz})$ were recorded for 30 minutes under resting conditions. Pregnancies with $\mathrm{CH}$ had a significantly elevated blood pressure compared to controls (CON, $111 \mathrm{mmHg}$ [105-132], CH, $140 \mathrm{mmHg}$ [132-148], $p<0.001)$. An increased heart rate was found in both groups during the second half of pregnancy. Consequently decreased HRV was observed but more pronounced in CON. There was a shift in the frequency bands indicated by an elevation of the low-to-high frequency ratio ( $\mathrm{LF} / \mathrm{HF}$ ) in both groups due to a decrease in HF. VLF increased exclusively in CON. Our data showed no significant difference in heart rate variability in pregnancies with $\mathrm{CH}$ and CON. Variations were detectable longitudinally in normal pregnancies but also, albeit to a smaller degree, in chronic hypertensive pregnant women. Thus, our data indicate that patients with long-term hypertension are still able to respond to physiological changes required during pregnancy.

Preeclampsia, a serious, pregnancy-specific disorder characterized by proteinuria and hypertension after the 20th week of gestation, is still the leading cause for maternal and neonatal morbidity and mortality and occurs in $3-5 \%$ of all pregnancies. Since the etiology and causative pathogenetic factors for this pregnancy complication are still unknown, an early risk assessment using Doppler sonography is aimed to improve clinical management and outcome. However, the positive predictive value of Doppler sonography is limited to approximately $30 \%$, since women with a disturbed uterine perfusion only partly develop one of the pregnancy complications as preeclampsia, pregnancy-induced hypertension $(\mathrm{PIH})$, or intrauterine growth retardation (IUGR). Therefore, in a recent study [Walther et al., 2006] we investigated 58 consecutive patients with uterine perfusion disturbance and 44 parallel recruited normal pregnancies who underwent a Doppler sonography in the second trimester of pregnancy (median 21 weeks, range [18-22] weeks) at the Department of Obstetrics and Gynecology, University of Leipzig. All pregnancies were singleton, and at the time of examination the women were healthy, normotensive, without clinical signs of cervical incompetence and on no medication. 
The uterine perfusion was defined as pathological if there was bilateral notching and/or if the mean pulsatility index (PI) of both arteries was greater than 1.45 (using Doppler sonography). After the Doppler examination, all women underwent noninvasive continuous blood pressure monitoring via finger cuff $(100 \mathrm{~Hz}$, Portapres device Model 2, BMITNO, Amsterdam, The Netherlands) to analyze HRV, BPV and BRS. All measurements were performed over 30 minutes under standardized resting conditions between 8 AM and 12 PM. From the Portapres recordings, time series of beat-to-beat intervals, of systolic as well as diastolic pressure values were extracted to analyze HRV, BPV and BRS. The Kruskal-Wallis test was applied to test intergroup differences of clinical parameters whereas MannWhitney $\mathrm{U}$ test was used to group according to differences in variability parameters. Forward stepwise linear discriminant analysis with a maximum of three parameters was used to estimate the group classification rates. The significance level was set at $p<0.05$. From 102 pregnancies 16 developed a preeclampsia. Independent of uterine perfusion, we investigated the general prediction of variability parameters for preeclampsia. Stepwise discriminant analysis identified three crucial parameters: "HF" high frequency in diastolic blood pressure, the number of tachycardic BRS in a range of 4 $6 \mathrm{~ms} / \mathrm{mmHg}$, the normalized very low frequency component in HRV, which discriminated with a sensitivity of $87.5 \%$, a specificity of $83.7 \%$ and a PPA of $50.0 \%$. However, in the subgroup with abnormal uterine perfusion, the same three parameters achieved a sensitivity of $93.7 \%$, a specificity of $85.7 \%$, and improved the PPA to $71.4 \%$ with a negative predictive accuracy (NPA) of $97.3 \%$ (see also Fig. 17).

Until now, a lot of effort has been made to develop a simple and clinically feasible test for early prediction of preeclampsia. However, previous studies using one-stage or two-stage doppler sonography, alone or combined with humoral, endothelial or cardiovascular parameters, did not achieve sufficient sensitivity and positive predictive value or were costly or invasive. Although parameters of HRV and $\mathrm{BPV}$ as well as BRS were initially developed for risk stratification in cardiology, the field of clinical applications broadened in recent years and these parameters of autonomic cardiovascular control are also influenced in hypertensive pregnancies disorders. Therefore, we tested the hypothesis whether this method is applicable for prediction of preeclampsia, since the preeclampsia-associated endothelial dysfunction could be recognized early by these parameters. While the incidence for preeclampsia in the western population is $3-5 \%$ of all pregnancies and a pathological uterine perfusion in the second trimester is known to be an indirect sign of an inadequate trophoblast but increases the positive predictive accuracy only to $30 \%$, we demonstrate here that three variability and BRS parameters allowed us to predict preeclampsia weeks before clinical manifestation with a PPA of $50.0 \%$. Since no other developed singular method reached higher predictability, our method consequently has higher PPA than all described independent humoral factors or singular

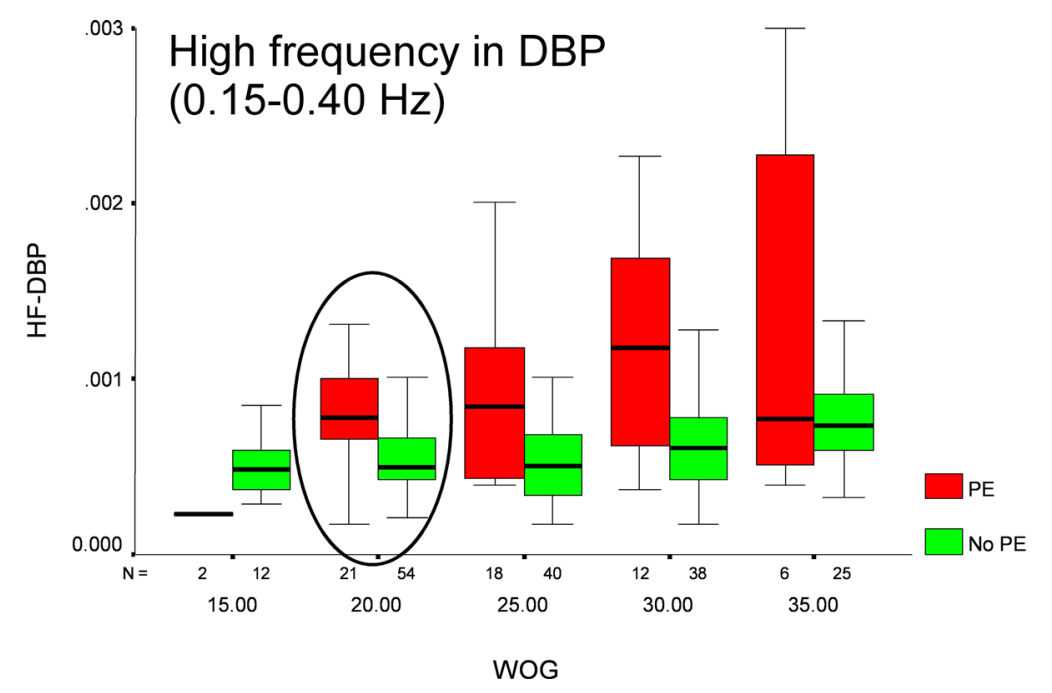

Fig. 17. The high frequency in diastolic blood pressure (HF-DBP) versus the week of gestation (WOG). Already at the 20th WOG there is a significant increase of HF-DBP in patients who later developed preeclampsia (PE). 
measured clinical parameters. But, when combined with the Doppler sonography of uterine arteries, we achieve the highest predictive level of all published noninvasive trials. Especially the high positive predictive accuracy of $71.4 \%$ provides clinical relevance to detect early pathological arterial stiffness. This is underlined by the fact that our approach of concurrent measurement of uterine perfusion and variability parameters is a feasible, inexpensive and noninvasive one-stop clinical assessment in the second trimester. Therefore, the actually most precise prediction of preeclampsia by concurrent measurement of uterine perfusion and calculation of cardiovascular beat-to-beat parameters in the second trimester may promote an early therapeutic strategy to reduce upcoming pathophysiological characteristics of the disease and consequently to prevent complications with high morbidity and mortality.

\subsection{Cardiovascular control in congestive heart failure}

The BRS is an important parameter in the classification of patients with reduced left ventricular function. Therefore, the aim of a special study [Malberg et al., 2002] was to investigate BRS in patients with dilated cardiomyopathy (DCM) and in healthy subjects (controls), as well to compare the values of BRS parameters with parameters of HRV and BPV. ECG, continuous blood pressure and respiration curves were recorded for 30 minutes in $27 \mathrm{DCM}$ patients and 27 control subjects. The Dual Sequence Method (DSM, cf. paragraph 2.4) includes the analysis of spontaneous fluctuations in systolic blood pressure and the corresponding beat-to-beat intervals of heart rate to estimate bradycardic, opposite tachycardic and delayed baroreflex fluctuations. The number of systolic blood pressure/beat-to-beat interval fluctuations in DCM patients was reduced in comparison with controls (DCM patients: male, $154.4 \pm$ $93.9 \mathrm{~ms} / \mathrm{mmHg} ;$ female, $93.7 \pm 40.5 \mathrm{~ms} / \mathrm{mmHg}$; controls: male, $245.5 \pm 112.9 \mathrm{~ms} / \mathrm{mmHg}$; female, $150.6 \pm 55.8 \mathrm{~ms} / \mathrm{mmHg}, p<0.05)$. The average slope in DCM patients was lower than in controls (DCM, $5.3 \pm 1.9 \mathrm{~ms} / \mathrm{mmHg}$; controls, $8.0 \pm$ $5.4 \mathrm{~ms} / \mathrm{mmHg} ; p<0.05)$. Discriminant function analysis showed, that in the synchronous range of the standard sequence method, the DCM and control groups could be discriminated with only $76 \%$ accuracy, whereas the DSM gave an improved accuracy of $84 \%$. The combination of six parameters of
HRV, BPV and DSM gives a classification accuracy of $96 \%$, whereas six parameters of HRV and BPV could separate the two groups to only $88 \%$ accuracy. Thus, DSM leads to an improved characterization of autonomous regulation in order to differentiate between DCM patients and healthy subjects. We found, that BRS in DCM patients is significantly reduced and apparently less effective.

New methods for the analysis of arrhythmias and their hemodynamic consequences have been applied in risk stratification, in particular to patients after myocardial infarction [Schmidt et al., 1999b]. However, the relation to cardiovascular variability parameters was unclear. Therefore, we conducted a study [Malberg et al., 2003; Malberg et al., 2004] in which we investigated the suitability of short-term heart rate turbulence (HRT) analysis in comparison to HRV and BPV as well as BRS analyses to characterize the regulatory differences between patients with DCM and healthy controls. In this study, 30 minutes' data of noninvasive continuous blood pressure and ECGs of 37 DCM patients and 167 controls measured under standard resting conditions were analyzed. The results show highly significant differences between DCM patients and controls in heart rate and blood pressure variability as well as in baroreceptor sensitivity parameters. Applying a combined heart rate-blood pressure trigger, ventricular premature beats were detected in $24.3 \%$ (9) of the DCM patients and $11.3 \%$ (19) of the controls. This fact demonstrates the limited applicability of short-term HRT analyses. However, the HRT parameters exhibited significant differences in this subgroup with ventricular premature beats (turbulence onset: DCM: $1.80 \pm 2.72$, controls: $-4.34 \pm 3.10, p<0.001$; turbulence slope: DCM: $6.75 \pm 5.50$, controls: $21.30 \pm 17.72, p=$ 0.021). Considering all (including HRT) parameters in the subgroup with ventricular beats, a discrimination rate between DCM patients and controls of $88.0 \%$ was obtained (max. 6 parameters). The corresponding value obtained for the total group was $86.3 \%$ (without HRT parameters). Although, premature beats were excluded before HRV analysis, the highest correlation of HRT to HRV parameters was 0.94 in controls and 0.87 in DCM patients. The results of this study indicated that HRV and HRT have at least the same prognostic value but HRV parameters have a significantly higher applicability. Moreover, we already showed in 1998 [Voss et al., 1998] that the predictive value of HRV using sophisticated parameters is significantly higher than 
the HRV index alone. We therefore think that the HRV parameters with the optimal predictive values as well as other ECG-based risk stratifiers should be retrospectively determined and then compared to HRT measurements. Our observations [Wessel et al., 2004c] cast doubt on the conclusion of Barthel et al. [2003] that HRT is the strongest ECG-based risk predictor.

In a "news and views" article of NATURE in 2002 Dante R. Chialvo [2002] has reported a new and promising approach by Madalena Costa et al. [2002] to distinguish healthy hearts from those suffering congestive heart failure (CHF) or atrial fibrillation (AF). The problem behind this is a subject of various attempts to characterize complex dynamics, such as consecutive beat-to-beat intervals of the heart. Costa et al. [2002] used the multiscale entropy MSE (cf. Sec. 2.3.6) which estimates the average uncertainty of such intervals; in other words MSE measures how strong the next beatto-beat interval will surprise us on average. Costa et al. [2002] showed that the heart-beat time series of healthy people asymptotically approaches a constant value of entropy as the measurement scale is increased, whereas heart beats of diseased hearts show a departure from this "constancy of surprise". They mainly conclude that healthy and pathologic groups can be consistently differentiated using MSE, while conventional algorithms fail. We would like to point out two major weaknesses of this study: Firstly, the age of the patients and the healthy subjects they considered is significantly different, and this is similar as to compare apples and oranges. Secondly, in contrast to their main statement, we show here that already a rather simple measure of complexity from symbolic dynamics [Kurths et al., $1995]$ is able to discriminate clearly between the CHF patients and even age-matched healthy subjects. Hence, no sophisticated techniques such as the MSE are necessary. We can concentrate in this tutorial on $\mathrm{CHF}$ patients because subjects with $\mathrm{AF}$ are characterized by a very broad sail-shaped beat-tobeat interval distribution, which is easily detectable by the standard deviation or the histogram [Tateno \& Glass, 2001] of the original signal.

(i) Age dependence: It is well-known in cardiology that HRV strongly depends on the age of the subjects; younger individuals have a significantly higher HRV than elderly ones [Weisfeldt et al., 1992]. Figure 1 of [Chialvo, 2002] or Fig. 3 of [Costa et al., 2002] show that it is easy to discriminate between healthy and CHF patients by means of their entropy

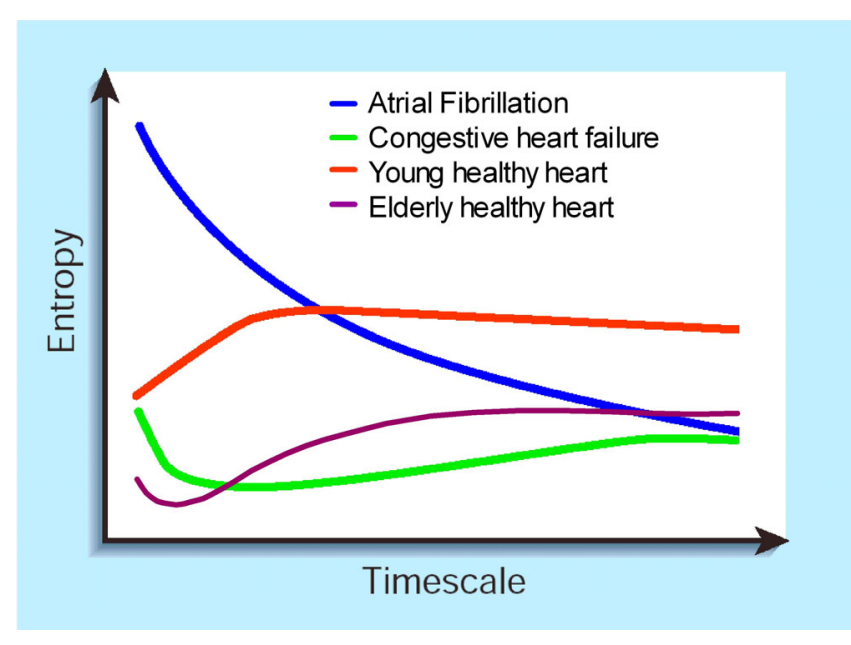

Fig. 18. Multiscale entropy values for the patients analyzed in [Costa et al., 2002] by combining their Figs. 3 and 4 .

measure. Additionally, Fig. 4 of [Costa et al., 2002] demonstrates that it is also possible to distinguish clearly between young and elderly volunteers. Simply combining Figs. 3 and 4 of [Costa et al., 2002] it immediately becomes clear that $\mathrm{CHF}$ patients and the elderly volunteers have approximately the same entropy values (cf. Fig. 18). Surprisingly, Costa et al. [2002] did not compare elderly healthy with elderly CHF groups, but compared young healthy subjects ( $32 \pm 6$ years) with elderly CHF patients $(56 \pm 11$ years [Goldberger et al., 2000]). This is indeed as to compare apples and oranges. So it can only be concluded from their analysis that the younger healthy heart surprises more on all scales than the elderly sick heart and, additionally, as the elderly healthy heart!

(ii) Simple discrimination method: Moreover, Chialvo [2002] stated that other approaches fail to detect such differences found in [Costa et al., 2002]. Next, we demonstrate that for discriminating healthy subjects from CHF patients no sophisticated multiscale entropy measure is necessary. We analyze the data of $15 \mathrm{CHF}$ patients (11 male, 4 female, age: $56 \pm 11$ ) and the $24 \mathrm{~h}$ time series of 18 young healthy persons (13 female, 5 male, age: $34 \pm 8$ ) available from Physionet [Goldberger et al., 2000]. To go beyond the apples and oranges comparison above, we additionally consider the beat-to-beat intervals of 15 healthy elderly subjects (11 male, 4 male, age: $56 \pm 5$ ). Three time series from the CHF group had more than $10 \%$ ectopy and were inserted into a CHF subgroup (CHF > 10\%). After preprocessing, standard time and frequency domain parameters were 
calculated from the time series. These parameters are based on linear techniques and statistically characterize HRV. To classify additionally dynamical changes, we use only one measure based on the concept of symbolic dynamics which has been recently successfully applied to other cardiological problems [Kurths et al., 1995; Wessel et al., 2000c]. The main finding in discriminating young healthy from CHF patients is that there are enormous discrepancies in heart rate regulation between both groups. Hence, almost all standard parameters already show highly significant differences. The best univariate parameter, we have found, is the simple measure POLVAR20 (cf. Eq. (10) in Chapter 2) based on symbolic dynamics [Wessel et al., 2000c], which detects intermittently decreased HRV (CHF: 0.68-0.90 inter-quartile range - IQR, young healthy: 0.05-0.13 IQR, $p<10^{-8}$, Mann-Whitney U-Test) and completely discriminates the young healthy and the CHF group. POLVAR20 characterizes short phases of low variability from successive symbols of a simple alphabet, consisting of only the symbols " 0 " and " 1 ". The elderly healthy persons show intermediate POLVAR20 values (0.210.42 IQR) which are significantly different to those of the younger healthy group $\left(p<10^{-4}\right)$ as well as different from the CHF patients $\left(p<10^{-5}\right)$. Plotting POLVAR20 versus the age of the investigated subjects demonstrates that the age-matched elderly healthy can be separated almost completely from the CHF group (Fig. 19; 93\% correctly classified for a cut-off of 0.5). Summarizing, the healthy heart surprises more than the sick heart. However, the level of surprise coming from a healthy heart decreases with age.

Finally, a correlation analysis between MSE and linear as well as nonlinear HRV parameters was performed. For all scales, high correlations between several HRV parameters and multiscale entropy were found. The closest connection, however, was found for MSE and the Shannon-entropy of the histogram ( $r>0.8$ for all scales) demonstrating that MSE measures the degree of randomness very similar to the simple Shannon-entropy. A comment, correcting the work of Costa et al. [2002], was published in Physical Review Letters [Wessel et al., 2003]. ${ }^{1}$

For HRV data it is well known that a metronomic heart rate is pathological; the healthy heart is influenced by multiple neural and hormonal factors

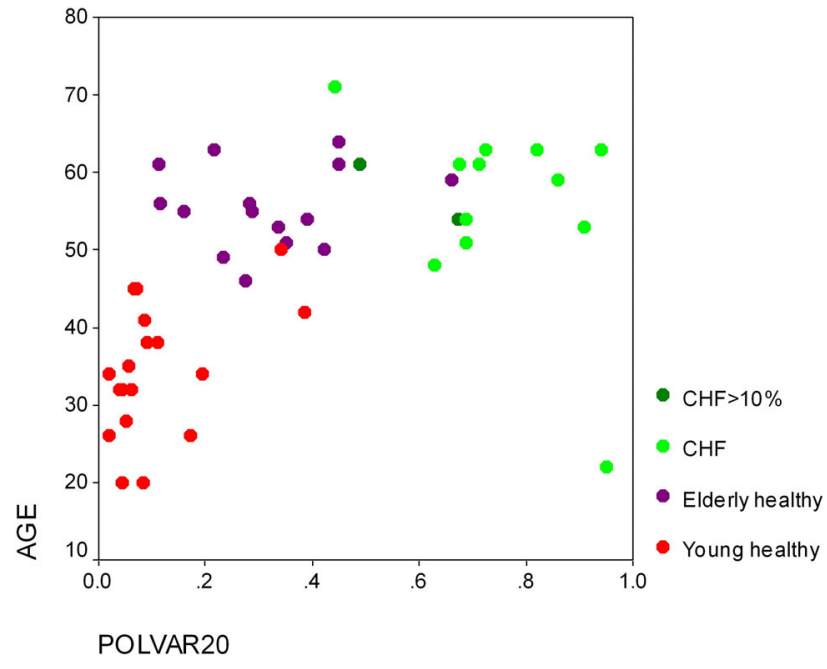

Fig. 19. Multiscale entropy values for the patients analyzed in [Costa et al., 2002] by combining their Figs. 3 and 4 .

that result in variations in RR-intervals. Even after three decades of study, new techniques continue to reveal properties of the time series of RR-intervals. Approximately one decade ago, first papers were published suggesting the fractal dimension [Osaka et al., 1993; Yamamoto \& Hughson, 1994b] as a promising measure for HRV data. For this reason, we applied this method to a group of 55 patients with dilated cardiomyopathy (DCM) and 55 healthy subjects as controls [Carvajal et al., 2005]. The 24-h $\mathrm{RR}$ time series for each subject was divided into segments of 10,000 beats to determine the correlation dimension (CD) per segment. A study of the influence of the time delay (lag) in the calculation of CD was performed. Good discrimination between both groups $(p<0.005)$ was obtained with lag values of 5 or greater. CD values of DCM patients $(8.4 \pm 1.9)$ were significantly lower than $\mathrm{CD}$ values for controls $(9.5 \pm 1.9)$. An analysis of $C D$ values of HRV showed that for healthy people, CD night values $(10.6 \pm 1.8)$ were significantly greater than CD day values $(9.2 \pm 1.9)$, revealing a circadian rhythm. In DCM patients, this circadian rhythm was lost and there were no differences between CD values in day $(8.8 \pm 2.4)$ and night $(8.9 \pm 2.1)$. One problem we recognized in our first analysis was to find a scaling region for HRV data to calculate the correlation dimension and to avoid pitfalls. Therefore, we developed a new method to solve this problem. The large-scale dimension density (LASDID, cf. Sec. 2.3.5) is estimated from the time series using a normalized Grassberger-Procaccia

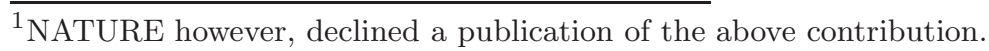


algorithm, which leads to a suitable correction of systematic errors produced by boundary effects in the rather large scales of a system. In this way, it is possible to analyze very short and nonstationary data, such as HRV. Moreover, this method allows us to analyze short parts of the data and to look for differences between day and night. In a recent paper, we reanalyzed the HRV data from the 2002 Computers in Cardiology Challenge (http://www.physionet.org/challenge/2002/) using the concept of LASDID and additionally applied this technique to data of healthy persons and of patients with cardiac diseases [Raab et al., 2005; Raab et al., 2006]. The circadian changes in the dimension density enabled us to distinguish almost completely between real data and computer generated data from Computers in Cardiology 2002 challenge using only one parameter. Furthermore, we analyzed the data of 15 patients with atrial fibrillation $(\mathrm{AF}), 15$ patients with congestive heart failure (CHF), 15 elderly healthy subjects (EH) as well as 18 young and healthy persons ( $\mathrm{YH}$ ). With our method we were able to separate completely the AF (LASDID: $0.97 \pm 0.02$ ) group from the others and, especially during daytime, the $\mathrm{CHF}$ patients show significant differences to the young and elderly healthy volunteers (CHF: $0.65 \pm 0.13$, $\mathrm{EH}: 0.54 \pm 0.05, \mathrm{YH}: 0.57 \pm 0.05, p<0.05$ for both comparisons). Moreover, for the CHF patients we found no circadian changes in LASDID (day: $0.65 \pm 0.13$, night: $0.66 \pm 0.12$, n.s.) in contrast to healthy controls (day: $0.57 \pm 0.05$, night: $0.67 \pm 0.07$, $p=0.00004)$. To the best of our knowledge, this is the first method which involves just one single parameter to accurately distinguish the considered groups from the 2002 Computers in Cardiology Challenge. Using the concept of large scale dimension densities, for the first time, a nearly perfect classification was performed. Finally, the application to data of different cardiac diseases demonstrated the quality of being appropriate for clinical use. $^{2}$

The main intention of a recent contribution [Wessel et al., 2006] was to test the hypothesis that nonlinear autoregressive model-based data analysis based on short-term heart rate variability improves the results of risk stratification. For this purpose, a nonlinear regression approach (cf. Sec. 2.6), namely, the maximal correlation method was applied to the data of 37 patients with dilated cardiomyopathy as well as of 37 age- and sex-matched healthy subjects. For simplicity reasons, we started with a linear autoregressive model of order $k$ given in Eq. (46).

$$
X_{j}=\sum_{i=1}^{k} a_{i} \cdot X_{j-i}+\eta .
$$

For different model orders $k$ up to 10, linear autoregressive modeling was performed. The model coefficients themselves were considered as parameters for discrimination between DCM and controls. The model coefficients however, were significant only up to order 2 (results not shown here). The residual variance $\eta$ was significantly lower in the DCM group, certainly caused by the lower HRV in this group. The absolute values of the coefficients $a_{1}$ and $a_{2}$ were higher for the controls, indicating a higher dependence from the two predecessors for healthy subjects. Based on the findings from linear autoregressive modeling, a nonlinear additive autoregressive (NAAR)-model of order 2

$$
X_{j}=\phi_{1}\left(X_{j-1}\right)+\phi_{2}\left(X_{j-2}\right)+\eta, \quad j=3, \ldots, n
$$

was investigated. For simplicity reasons, the lefthand side of Eq. (47) was set to the identity and we were interested in estimating the functional dependence of $X_{j}$ on its two predecessors. Therefore, the maximal correlation method was used to estimate the possibly nonlinear dependencies (as optimal transformations) nonparametrically. To quantify these relations, a third order polynomial fitting of the optimal transformations was performed (cf. Fig. 20). The fitting coefficients themselves (from Eq. (48)) were considered as the parameters in cardiac diagnostics. Using higher orders for polynomial fitting turned out not to be useful in this study.

$$
\begin{gathered}
X_{j}=a_{1} \cdot X_{j-1}^{3}+a_{2} \cdot X_{j-1}^{2}+a_{3} \cdot X_{j-1}+a_{4} \\
+b_{1} \cdot X_{j-2}^{3}+b_{2} \cdot X_{j-2}^{2}+b_{3} \cdot X_{j-2}+b_{4} \\
j=3, \ldots, n .
\end{gathered}
$$

Figure 20 visualizes the polynomial fitting of $\phi_{1}$ and $\phi_{2}$ from Eq. (47) based on group averages. While the optimal transformations $\phi_{1}$ are similar for the DCM and the control group (except the small difference at larger beat-to-beat intervals), the behavior of $\phi_{2}$ is totally different in both groups. The coefficients $b_{1}$ and $b_{2}$, which represent the quadratic and the cubic parts of the fitting and are, therefore, lower than $b_{3}$ and $b_{4}$, are both higher

\footnotetext{
${ }^{2}$ Our contribution was the winning paper of the Computers in Cardiology Challenge 2005.
} 

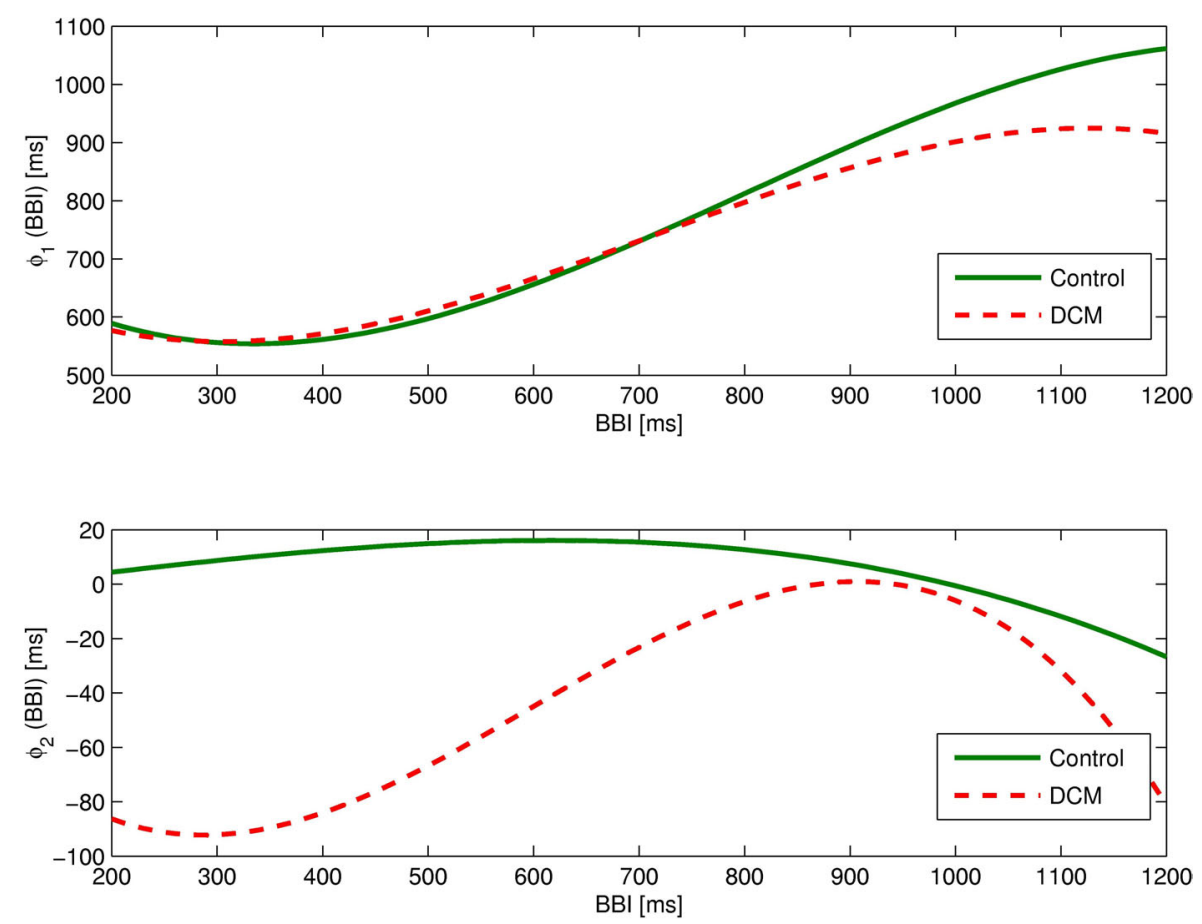

Fig. 20. Averaged polynomials of the optimal transformations $\phi_{1}$ and $\phi_{2}$ for the DCM (dashed line) as well as for the control group (solid line). (BBI beat-to-beat interval.)

in absolute value for the DCM subjects (data not shown here) and lead to more oscillating behavior of the fitting for this group. The coefficient $b_{3}$, representing the linear part of the fitting, is $b_{3}=0.045$ for the control subjects and, hence, higher than $b_{3}=-0.61$ for the DCM group. This means that we have a linear tendency for a slight increase of $\phi_{2}$ with increasing beat-to-beat interval, whereas, the DCM group exhibits a clear decrease. The dominant part for the discrimination of both groups, however, is the quadratic term $b_{2}$ which is extremely higher in the DCM group. Thus, for the interpretation of these results all fitting coefficients have to be considered together.

We found that this approach is a powerful tool in discriminating cardiac patients from healthy controls - one model parameter discriminates best in comparison to all other parameters in this study and promising for further model-based analyses.

\subsection{Autonomic control after cardiac surgery}

Impairment of the baroreceptor reflex activity reflects an alteration of the autonomous regulation of the cardiovascular system and has proven to precede fatal outcome in patients after acute myocardial infarction. For that reason, a pilot study was performed [Bauernschmitt et al., 2004] to analyze baroreceptor sensitivity, heart rate variability and blood pressure variability in patients early after coronary surgery. 25 male patients undergoing coronary artery bypass surgery were examined; normal values were obtained from healthy volunteers. Arterial pressure signals were recorded from a radial artery catheter for 30 minutes preoperatively and in short intervals after surgery. Mechanical manipulations and pharmacological interventions were avoided during the sampling periods. Baroreflex function was calculated according to the Dual Sequence Method, heart rate variability and blood pressure variability were calculated including nonlinear methods. Initial values of the patients did not differ from healthy volunteers. The strength of baroreflex sensitivity (increases of blood pressure causing a synchronous decrease of heart rate) is low 2 hours postoperatively. The number of delayed tachycardic changes of heart rate, which are caused by sympathetic activation, is only moderately reduced when compared to values obtained from healthy volunteers. Heart rate variability is widely unchanged as compared to preoperative values; blood pressure variability showed an increase of low-frequency components, again indicating sympathetic predominance. Nonlinear analyses revealed reduced system complexity at the beginning of the 
postoperative course. Obviously, there is a vagal suppression 20 hours after surgery, while the sympathetic tonus works in a normal range. This unbalanced interaction of the autonomous system is similar to findings in patients after myocardial infarction. The predictive value of these markers has to be elucidated in further clinical studies.

An important problem is that atrial fibrillation (AF) occurs in 20-40\% of patients after open heart surgery, leading to increased morbidity and prolonged hospital stay. Earlier studies have demonstrated, that depressed baroreflex function predicts mortality and major arrhythmic events in patients surviving myocardial infarction. Cardiac surgery per se leads to decreased BRS and HRV, so the aim of the present study [Bauernschmitt et al., 2007] was to analyze the impact of the cardiovascular autonomous system on the development of post surgical AF. Fifty-one consecutively included patients undergoing aortic valve replacement, coronary artery bypass surgery or combined procedures were included. Noninvasive blood pressure and ECG were recorded the day before and $24 \mathrm{~h}$ after surgery. BRS, HRV and nonlinear dynamics were calculated using standard methods. Eighteen patients developed AF during the first postoperative week, while 33 remained in sinus rhythm throughout the observation period. Patients with postoperative AF showed a significantly reduced bradycardic BRS prior to surgery. In both groups, surgery caused a decrease of BRS and HRV. Analysis of nonlinear dynamics revealed a tendency towards decreased system complexity caused by the operation; this trend was more pronounced in patients remaining in sinus rhythm. Patients experiencing postoperative AF obviously suffer from impaired baroreflex sensitivity present already before surgery. These findings may be used to guide prophylactic antiarrhythmic therapy.

\subsection{Cardiovascular control in animal models}

HRV is a relevant predictor of cardiovascular risk in humans. A significant genetic influence on HRV is suggested [Kupper et al., 2004], although the genes involved are ill-defined. The Mas-protooncogene encodes a G-protein-coupled receptor with seven transmembrane domains highly expressed in testis and brain. Since this receptor is supposed to interact with the signaling of angiotensin II, which is an important regulator of cardiovascular homeostasis, heart rate and blood pressure were analyzed in Mas-deficient mice. Using a femoral catheter the blood pressure of mice was measured for a period of 30 minutes and 250 data values per second were recorded. The mean values and range of heart rate and blood pressure were then calculated. Neither heart rate nor blood pressure were significantly different between knockout mice and controls. However, high resolution recording of these parameters and analysis of the data by nonlinear dynamics revealed significant alterations in cardiovascular variability in Mas-deficient animals. In particular, females showed a strong reduction of HRV. Furthermore, the data showed an increased sympathetic tone in knockout animals of both genders. The marked alterations detected in Mas-deficient mice of both genders suggest that the Mas-protooncogene is an important determinant of heart rate and blood pressure variability.

The analysis of the cardiac autonomic regulation in patients with familiar hypertrophic cardiomyopathy (FHC) revealed controversial results. In order to investigate the role of autonomic dysfunction in the pathogenesis of $\mathrm{FHC}$, transgenic rats overexpressing either disease causing mutation Asp175Asn or Glu180Gly in a-tropomyosin (TPM1) were studied here for changes in hemodynamic parameters and baroreflex regulation in comparison to transgenic and nontransgenic controls by telemetry [Wernicke et al., 2007]. HRV and BPV were analyzed using time- and frequency domain, as well as nonlinear measures. The Dual Sequence Method was used for the estimation of the spontaneous baroreflex regulation. In transgenic rats with either TPM1 mutation, changes in cardiac autonomic control were detected during exercise, but not at rest. In transgenic rats carrying mutation Asp175Asn reduced total HRV was observed based on the determination of RMSSD $(p<0.01)$. In comparison, animals with mutation Glu180Gly demonstrated increased sympathetic nervous activity, as evidenced by a ten-fold elevation of the low frequency component and an increased low/high frequency component ratio $(p<0.01)$. In animals with either TPM1 mutation, a strong increase in baroreflex activity was observed to keep blood pressure and BPV parameters normal. While baroreflex slopes remained unchanged, the frequency of bradycardic and tachycardic fluctuations was elevated 1.5-fold and 4.0-fold in rats carrying mutation Asp175Asn and Glu180Gly, respectively $(p<$ 0.001). Therefore, individual mutations in TPM1 
differently affect cardiac autonomic regulation. The analysis of cardiac autonomic control, particularly of baroreflex regulation, might represent a noninvasive approach for early diagnosis and the assessment of the clinical course of FHC.

\subsection{Heritability of heart rate variability}

We tested the hypothesis, that genes have an influence on HRV [Voss et al., 1996a]. This genetic influence was assessed in 62 twin pairs (30 monozygotic - MZ, 32 dizygotic — DZ). From all twins, long-term electrocardiographic records were obtained, edited, and analyzed. HRV analysis was performed on the basis of parameters from timedomain, frequency-domain, and nonlinear dynamics. First, the parameter distances between the two twins of a pair and between one of the two and a third randomly selected person of another age-matched twin pair (ST1) were compared. Second, the parameter distances between the two twins and the averaged parameter distances of these two twins to all other age-matched persons (ST2) were compared. Finally, the averaged differences in parameter values between monozygotic and dizygotic age-matched twin pairs were compared. For statistical analysis, the nonparametric Wilcoxon's matched-pair signed rank test and parametric $t$-test for paired samples were applied. We find that twin pairs show a significant lower difference in parameter values than other randomly selected and age-matched couples $(p<0.001$ in ST1 and ST2). This reflects a considerable familial influence. Most parameters of the time-domain, none of the frequency-domain, and half of the nonlinear dynamics show significant differences between twin pairs and nontwin pairs. As a result of the comparison between monozygotic and dizygotic twin pairs, a significant lower parameter difference in the monozygotic pairs $(p<0.05)$ is found. These results suggest that there is a genetic component in heart rate generation and HRV, in addition to family environmental influences. Analysis of HRV might become a useful method in phenotyping severe genetic changes in cardiovascular diseases.

Decreased HRV is associated with congestive heart failure, post-myocardial infarction, ventricular arrhythmias, sudden cardiac death, and advancing age. A deletion/insertion polymorphism in the angiotensin-converting enzyme gene and a substitution (M235T) in the angiotensinogen gene have been associated with risk for heart disease. The aim of our study [Busjahn et al., 1998] was to determine the heritability of HRV and related parameters in monozygotic and dizygotic twins and to assess the influence of angiotensin-converting enzyme and angiotensinogen polymorphisms. We studied $95 \mathrm{MZ}$ pairs and $46 \mathrm{DZ}$ pairs. We measured HRV and related parameters, angiotensin-converting enzyme and angiotensinogen levels, plasma norepinephrine, angiotensin-converting enzyme, and angiotensinogen genotypes. We found that HRV and related parameters were significantly influenced by genetic variability, although nonshared genetic effects were also important. Angiotensinogen and plasma norepinephrine were generally correlated with decreased HRV, whereas angiotensin-converting enzyme was correlated with perturbations of normal rhythmic HRV. Nevertheless, the DD angiotensin-converting enzyme genotype was associated with increased HRV $(p<0.05)$, whereas angiotensinogen polymorphisms had no effect. We conclude that HRV and related parameters are in part heritable. Interestingly, the DD angiotensin-converting enzyme genotype is associated with increased HRV.

\subsection{Further applications}

Approximate Entropy (ApEn, cf. Sec. 2.3.6) as a measure of randomness provides some interesting information on abnormalities in heart rate behavior in relation to cardiac disorders. Decreased ApEn values of heart beat fluctuations have been found to precede spontaneous episodes of atrial fibrillation (AF) in patients without structural heart disease [Vikman et al., 1999]. In this study, a progressive decrease in ApEn, indicating reduced complexity of heart rate dynamics, was observed before the onset of AF episodes. Furthermore, the values of ApEn before the onset of AF were lower compared with the values obtained from matched healthy control subjects. In a more recent study [Vikman et al., 2005] these results could be confirmed, however a more predictive parameter for $\mathrm{AF}$ was found: the turbulence onset (TO). TO was significantly less negative during the $1 \mathrm{~h}$ preceding $\mathrm{AF}$ than during the non-AF hours $(0.71 \pm 1.76$ versus $-0.35 \pm 1.46, p<0.00001)$. Less negative TO before AF was observed among both, patients with structural heart disease $(1.16 \pm 1.73$ versus $0.07 \pm 1.23$; $p<0.0001)$ and patients with lone $\mathrm{AF}(0.17 \pm 1.67$ versus $-0.85 \pm 1.56 ; p<0.0001)$. No significant difference was seen in the turbulence slope between 
the AF and control, and none of the traditional frequency and time domain measures differentiated between both; ApEn was significantly lower before AF than during the non-AF hours $(p<0.01)$. Increased predictability in heart rate behavior, measured by ApEn, also has been reported to precede spontaneous episodes of ApEn after coronary artery bypass surgery [Hogue, Jr. et al., 1998] Analysis of HRV in this study was performed in three sequential 20-minute intervals preceding the onset of postoperative AF (24 episodes in 18 patients). These data were compared with corresponding intervals in 18 sex- and age-matched postoperative control subjects who did not develop AF. The authors could show that increased heart rate and decreased ApEn were independently associated with AF. We have to note here, that we are able to detect patients with high risk for postoperative AF already at the preoperative state using baroreflex sensitivity measurements (see [Bauernschmitt et al., 2007]). Although reduced complexity in heart dynamics has been associated with postoperative complications after vascular surgery [Fleisher et al., 1993], there is no clear evidence that the changes in complexity of heart rate behavior are related to the risk for death. However, recent data [Perkiomaki et al., 2003] suggest that increased temporal complexity of repolarization predicts mortality in high risk ICD patients with decreased left ventricular function. ApEn also appears to have broad application to hormone pulsatility analysis within endocrinology [Pincus, 2000], bringing a new perspective to the assessment of secretory patterns. ApEn seems to be complementary to pulse detection algorithms widely employed to evaluate hormone secretion time-series. Pincus states, that ApEn is applicable to systems with at least 50 data points and to broad classes of models: It can be applied to discriminate both general classes of correlated stochastic processes, as well as noisy deterministic systems. Sample Entropy (SampEn) as a refined ApEn measure was applied to a dense array electroencephalogram signals [Ramanand et al., 2004]. The objective of this study was to gain insight into complexity variations related to changing brain dynamics for electroencephalogram recorded from the three cases of passive, eyes closed condition, a mental arithmetic task and the same mental task carried out after a physical exertion task. It is observed that SampEn is a robust quantifier of complexity suited for short physiological signals such as the electroencephalogram and it points to the specific brain regions that exhibit lowered complexity during the mental task state as compared to a passive, relaxed state. In the case of mental tasks carried out before and after the performance of a physical exercise, SampAn can detect the variations brought in by the intermediate fatigue inducing exercise period. This enhances its utility in detecting subtle changes in the brain state that can find wider scope for applications in electroencephalogram based brain studies. Further studies showed applications of SampEn for heart rate recovery after exercise [Javorka et al., 2002], for heart rate dynamics in schizophrenic subjects [Kim et al., 2004] as well as the quantification of neonatal heart rate before the clinical diagnosis of sepsis [Cao et al., 2004]. Occasionally, traditional complexity measure have yielded contradictory findings when applied to real-world datasets obtained in health and disease states. The multiscale entropy (MSE) method [Costa et al., 2002, 2005] was suggested to overcome these contradictions. The authors applied the MSE methods to the fluctuations of the human heartbeat under physiologic and pathologic conditions. The method consistently indicates a loss of complexity with aging, with an erratic cardiac arrhythmia (atrial fibrillation), and with a life-threatening syndrome (congestive heart failure). Further, these different conditions have distinct MSE curve profiles, suggesting diagnostic uses. The authors conclude a general "complexity-loss" theory of aging and disease. Moreover, they applied the MSE method to the analysis of coding and noncoding DNA sequences and find that the latter have higher MSE, consistent with the emerging view that so-called "junk DNA" sequences contain important biological information. However, detrended fluctuation analysis also reveals a loss of heart rate dynamics in patients with heart failure. Altered fractal correlation properties have been associated with mortality in heart failure patients [Ho et al., 1997]. The investigation of a relatively large population with CHF suggests that reduced short-term scaling exponent is closely related to the risk of mortality [Makikallio et al., 2001a]. The short-term scaling exponent also has been suggested to be a specific risk marker of cardiac death in the elderly [Makikallio et al., 2001b]. As with ApEn, altered short-term fractal-like scaling properties of HRV have been observed to precede the spontaneous onset of AF among patients without a structural heart disease [Vikman et al., 1999]. Moreover, the short-term scaling exponent has been shown to change towards more random 
directions in ectopic tachycardia reflecting disturbances in autonomic regulation or in ectopic atrial pacemakers per se [Huikuri et al., 1999]. Using the Poincaré plot technique HRV was compared in 30 subjects receiving external Qi therapy (EQT) or placebo control therapy, in a crossover design experiment [Lee et al., 2005]. Subjects who received the EQT reported more pleasant and calm emotions than did the placebo group. Qi therapy reduced the heart rate and increased HRV as indicated by a reduced low frequency/high frequency power ratio of HRV. The Poincaré plot index of HRV and approximate entropy was greater in the EQT group than in the control group. These findings suggest that EQT stabilizes the sympathovagal function and cardiac autonomic nervous system by inducing more positive emotions than the placebo therapy. In conclusion, EQT may act by stabilizing both the autonomic nervous system and the emotional state. The latter study demonstrated the great opportunity for traditional Chinese therapy for the human cardiovascular system.

Another important issue is the impact of respiration, especially the breathing pattern variability. In [Bien et al., 2004] it was tested whether it can serve as a potential weaning predictor for postoperative patients recovering from systemic inflammatory response syndrome. Seventyeight mechanically ventilated patients who had undergone abdominal surgery were included when they were ready for weaning. They were divided into success $(n=57)$ and failure $(n=21)$ groups based upon their weaning outcome. Successful weaning was defined as patients free from the ventilator for over $48 \mathrm{~h}$, whereas a weaning failure was considered as reinstitution of mechanical ventilation within $48 \mathrm{~h}$ of extubation. Poincaré plot parameters in the failure group were significantly lower than those in the success group. The author concluded, that small breathing pattern variability is associated with a high incidence of weaning failure in postoperative patients with such a disease, and this variability may potentially serve as a weaning predictor. The important impact of respiration to cardiovascular regulation is summarized in the following review [Bernardi et al., 2005]. Therein it is stated, that respiration is a powerful modulator of HRV, and of baro- or chemoreflex sensitivity. This regulation occurs via a mechanical effect of breathing by synchronizing all cardiovascular variables at the respiratory rhythm (cf. also [Schafer et al., 1998]), particularly at a slow rate coincident with the Mayer waves in arterial pressure (approximately 6 cycles/min). The authors could show that slow breathing increases baroreflex sensitivity and reduces chemoreflex sensitivity, leading to increased parasympathetic and reduced sympathetic activity. The opposite can be seen during mental stress tests. Thus, appropriate modulation of breathing, can improve/restore autonomic control of cardiovascular and respiratory systems in relevant diseases such as hypertension and heart failure, and might therefore help improve exercise tolerance, quality of life, and ultimately, survival.

\section{Conclusion and Outlook}

Allegorically, the human heart may be compared with the engine of a car - both make bodies move. Although unnoticed, the healthy heart continuously provides oxygen supply. However, by doing so, it beats irregularly in contrast to the regular work of a car engine. The intervals between heart beats widely fluctuate and the diagnostic value of this complex variability has been an important challenge for physicians, biologists and, more recently, also for physicists. During the last decades it was shown that tools from linear system theory can provide valuable information. More recently, tools from nonlinear dynamics substantially improved the diagnostic value beyond that achieved by using solely linear parameters. However, there are still important challenges in understanding the dynamics of diseases intimately linked to the challenge of discovering the general principles that govern complex biological processes. Despite statistical data suggesting the predictive power of various cardiovascular indexes, none of these methods is in widespread clinical use at the moment. Therefore, more clinical studies using new and traditional methods are needed, before a clinical applicability can be definitely established.

Cardiovascular diseases are the main cause of death in western industrialized countries. With regard to recent social developments, there is an extraordinary demand for new computer-controlled diagnostic techniques to obtain a differentiated picture of the possibly damaged heart and to develop new biomedical devices for risk stratification. Cardiovascular variability analyses as well as body surface potential mapping and cardiac magnetic field mapping represent diagnostic tools which have recently made substantial progress. All methods try to identify new physiological information which may 
be used for diagnostics, prognosis and risk stratification, for clinical treatment and for pharmacologic or genetic research. One basic issue in all cardiovascular variability studies is the choice of a suitable measuring technique and the connected processing unit. A task force on HRV for the achievement of comparable results was established in 1996 [Task Force Heart Rate Variability, 1996]. The created guidelines became generally accepted, however, they suffer from three major limitations:

(i) The first limitation is that a $100 \mathrm{~Hz}$ sampling rate is sufficient. This is not true. We have shown [Voss et al., 1996c] that especially for high risk patients the relative error due to low sampling rate amounts to $15 \%$. We propose a sampling frequency of at least $500 \mathrm{~Hz}$ [Malberg \& Wessel, 2006].

(ii) The second limitation is the recommendation for manual editing of the beat-to-beat time series. The task force states, that automatic filters which exclude some intervals from the original sequence (e.g. the $20 \%$ rule) should not replace manual editing "as they are known to behave unsatisfactorily and to have undesirable effects leading potentially to errors" [Task Force Heart Rate Variability, 1996]. In our experience, the manual editing of the time series produces even more serious errors. Using a sophisticated preprocessing algorithm, it is now possible to analyze automatically large HRV data bases with big success (cf. [Voss et al., 1998]). However, obviously even sophisticated algorithms may produce errors and cannot compete with an experienced observer.

(iii) The third limitation is that no recommendations for nonlinear analyses are given. This tutorial gives an overview of linear as well as nonlinear methods, compares them and can therefore serve as a reference especially for nonlinear analyses. These nonlinear methods can lead to a substantial improvement of reliable diagnostics, because they are better suited to characterize the inherent nonlinear regulation. We have shown that a combination of linear and nonlinear approaches leads to a significantly improved classification rate.

In addition to the HRV analysis, lately morphologic ECG parameters were established. Therefore, we expect that the combination of methods introduced in this tutorial with parameters of the T-wave and of the QT-time will improve the classification rates in risk stratification. Today, the development of ECG techniques undergoes a renaissance with fast technological progress. In the future, longterm Holter ECGs have to include more and more nonlinear variability, morphologic ECG as well as coupling parameters (e.g. cardiorespiratory synchronization). Knowledge based systems will help to assign sophisticated parameters to physiological phenomena enabling automatic text generation for medical diagnostics. Moreover, automatic analyses of cardiovascular regulation in relation to physical activity and circadian variability will be possible. A further approach, which could be implemented in new devices, is the HRT method analyzing only ventricular premature beats and the behavior of the surrounding normal beats. This method should have significantly extended the variability analysis in myocardial infarction patients. However, we could show that in combination with linear and nonlinear methods a diagnostic increase can hardly be reached. Analyzing the variability of the systolic and diastolic blood pressure is a supplementary approach of cardiovascular characterization. With regard to clinical use, this is a relatively complex and expensive procedure applied only in research hospitals except from invasive measurements in intensive care units. Applying similar parameters as for HRV analyses, independent diagnostic information could be won. The analysis of the spontaneous BRS in research is an established and meaningful procedure for the analysis of cardiovascular regulation. This method investigates the heart rate reflex from instinctive blood pressure changes. If this reflex arises frequently and sufficiently strong it can serve as a diagnostic and prognostic positive indicator. Despite its enormous diagnostic power, the spontaneous BRS analysis could not yet be established routinely. The problem for clinical application of this method is again the high expense for a suitable blood pressure measurement device. All these methods from cardiovascular physics led to rapid developments in medicine. However, the transfer of scientific results into technical devices is problematic because of the very expensive and clinically extensive evaluation. On the other hand, the increasing occurrence of cardiovascular diseases and the reduction of healthcare expenses lead to an increasing demand on new therapeutical tools which should fulfill the following requirements:

- cost saving,

- preferably noninvasive nature,

- minimization of the patient's risk,

- capability of home care,

- higher diagnostic power. 
Therefore, devices have to be developed which are able to convey the same information contained in the blood pressure signals but which are remarkably cheaper.

The field of intensive care unit monitoring will be strongly changed by including cardiovascular physics parameters. Monitoring systems will enable an early prediction and prevention of serious cardiovascular events and lead to better recovering prognoses after serious medical interventions. The biosignals monitored in the intensive care unit will be analyzed with regard to special autonomous vegetative regulation processes. Some exemplary regulation processes which can be characterized via cardiovascular physics parameters are the baroreflex, the chemoreflex, the sympathetic blood vessel tone and sleep stage changes. To improve the medical significance, univariate analyses of the monitoring signals will be included in these stratifications. These univariate analyses comprise methods from time- and frequency domain as well as methods from nonlinear dynamics. Using multivariate model-based data analyses the medical staff will be provided with additional information about the current state of the patient which is not available today. The information about the autonomous regulation will be used in combination with clinical parameters, such as medication, age, gender, concomitant diseases for recovering prognoses and risk stratification. In this way, an improved recovery prognosis is possible enabling a minimization of patients risk, an individual intensive care therapy as well as a reduction of healthcare expenses.

Important developments are also to be expected for the model based methods. These approaches on the one hand aim at modeling one or more regulation processes, and on the other hand provide model parameters which should be used to answer clinically relevant questions. In this tutorial we have introduced an approach which is very promising in data-driven modeling and modelbased data analysis [Wessel et al., 2000b; Wessel et al., 2004a; Wessel et al., 2004b; Wessel et al., 2006]. We have found that the maximal correlation method is a powerful tool for medical data analyses and for solving mechanical engineering problems. This general method is therefore promising for further model-based analyses not only in cardiovascular physics. There is an enormous demand for the latter field also in pharmacological and genetic research, because interactions of different genotypes and pharmaceutics with cardiovascular regulation are almost unknown. Insofar, the further development of model based approaches represents an important contribution for new therapeutical therapies. One promising example is our idea of a circadian ICD for an individual prediction of life-threatening events (International patent registration PCT/DE2005/002040).

We could demonstrate in this tutorial, that data analyses and modeling methods from cardiovascular physics lead to significant improvements in different medical fields. Patients as well as the whole society would benefit from a rapid use of these potentials in clinical practice.

\section{Acknowledgments}

This work is the result of an interdisciplinary cooperation between different groups in several scientific fields. We thank all our colleagues. The authors acknowledge financial support by the EU Network of Excellence Grant No. NoE 005137 BioSim as well as by the Deutsche Forschungsgemeinschaft (BA 1581/4-1, BR 1303/8-1, BR 1303/10-1, KU 837/201, KU 837/23-1, PE 628/4-1).

\section{References}

Abarbanel, H. D. I., Brown, R. \& Kennel, M. B. [1991] "Lyapunov exponents in chaotic systems: Their importance and their evaluation using observed data," Int. J. Mod. Phys. B 5, 1347-1375.

Akselrod, S., Gordon, D., Ubel, F. A., Shannon, D. C., Barger, A. C. \& Cohen, R. J. [1981] "Power spectrum analysis of heart rate fluctuation: A quantitative probe of beat-to-beat cardiovascular control," Science 213, 220-222.

Alberte, C. \& Zipes, D. P. [2003] "Use of nonantiarrhythmic drugs for prevention of sudden cardiac death," J. Cardiov. Electrophysiol. 14, S87-S95.

Almog, Y., Eliash, S., Oz, O. \& Akselrod, S. [1996] "Nonlinear analysis of bp signal: Can it detect malfunctions in bp control?" Am. J. Physiol. Heart Circul. Physiol. 40, H396-H403.

Argyris, J. H., Faust, G. \& Haase, M. [1994] An Exploration of Chaos (North Holland, Amsterdam).

Babloyantz, A. \& Destexhe, A. [1988] "Is the normal heart a periodic oscillator?" Biol. Cybern. 58, 203211.

Bak, P., Tang, C. \& Wiesenfeld, K. [1987] "Selforganized criticality: An explanation of the $1 /$ f noise," Phys. Rev. Lett. 59, 381-384.

Barron, H. V. \& Lesh, M. D. [1996] "Autonomic nervous system and sudden cardiac death," J. Am. Coll. Cardiol. 27, 1053-1060. 
Barthel, P., Schneider, R., Bauer, A., Ulm, K., Schmitt, C., Schömig, A. \& Schmidt, G. [2003] "Risk stratification after acute myocardial infarction by heart rate turbulence," Circulation 108, 1221-1226.

Baselli, G., Cerutti, S., Civardi, S., Malliani, A. \& Pagani, M. [1988] "Cardiovascular variability signals: Towards the identification of a closed-loop model of the neural control mechanisms," IEEE Trans. Biomed. Eng. 35, 1033-1046.

Bauernschmitt, R., Malberg, H., Wessel, N., Kopp, B., Schirmbeck, E. U. \& Lange, R. [2004] "Impairment of autonomic control in patients early after cardiac surgery," Eur. J. Cardiothorac. Surg. 25, 320-326.

Bauernschmitt, R., Malberg, H., Wessel, N., Brockmann, G., Zinser, C., Eichinger, W. M., Bretthauer, G., Kurths, J. \& Lange, R. [2007] "Autonomic control in patients experiencing atrial fibrillation after cardiac surgery," Pace 30, 77-84.

Bernardi, L., Porta, C., Spicuzza, L. \& Sleight, P. [2005] "Cardiorespiratory interactions to external stimuli," Arch. Ital. Biol. 143, 215-221.

Berntson, G. G., Quigley, K., Jang, J. F. \& Boysen, S. T. [1990] "An approach to artifact identification: Application to heart period data," Psychophysiology 27, 586-598.

Berntson, G. G., Bigger, J. T., Ckberg, D. L., Grossman, P., Kaufmann, P. G., Malik, M., Nagaraja, H. N., Porges, S. W., P., S. J., Stone, P. H. \& van der Molen, M. W. [1997] "Heart rate variability: Origins, methods, and interpretative caveats," Psychophysiology 34, 623-648.

Bien, M. Y., Hseu, S. S., Yien, H. W., Kuo, B. I., Lin, Y. T., Wang, J. H. \& Kou, Y. R. [2004] "Breathing pattern variability: A weaning predictor in postoperative patients recovering from systemic inflammatory response syndrome," Intensive Care Med. 30, 241247.

Bigger Jr, J. T., Fleiss, J. L., Steinman, R. C., Rolnitzky, L., Kleiger, R. E. \& Rottman, J. N. [1992] "Frequency domain measures of heart period variability and mortality after myocardial infarction," Circulation $\mathbf{8 5}$, 164-171.

Breiman, L. \& Friedman, J. [1985] "Estimating optimal transformations for multiple regression and correlation," J. Am. Stat. Assoc. 80, 580-619.

Breithardt, G., Cain, M., el Sherif, N., Flowers, N., Hombach, V., Janse, M., Simson, M. \& Steinbeck, G. [1991] "Standards for analysis of ventricular late potentials using high resolution or signal-averaged electrocardiography. A statement by a task force committee between the European society of cardiology, the American heart association and the american college of cardiology," Eur. Heart J. 12, 473-480.

Brennan, M., Palaniswami, M. \& Kamen, P. [2001] "Do existing measures of poincar plot geometry reflect nonlinear features of heart rate variability?" IEEE Trans. Biomed. Eng. 48, 1342-1347.

Bristow, J. D., Honour, A. J., Pickering, G. W., Sleight, P. \& Smyth, H. S. [1969] "Diminished baroreflex sensitivity in high blood pressure," Circulation 39, 48-54.

Brown, R., Bryant, P. \& Abarbanel, H. D. I. [1991] "Computing the lyapunov spectrum of a dynamical system from an observed time series," Phys. Rev. A 43, 2787-2806.

Busjahn, A., Voss, A., Knoblauch, H., Knoblauch, M., Jeschke, E., Wessel, N., Bohlender, J., McCarron, J., Faulhaber, H., Schuster, H., Dietz, R. \& Luft, F. C. [1998] "Angiotensin-converting enzyme and angiotensinogen gene polymorphisms and heart rate variability in twins," Am. J. Cardiol. 81, 755760 .

Cao, H., Lake, D. E., Griffin, M. P. \& Moorman, J. R. [2004] "Increased nonstationarity of neonatal heart rate before the clinical diagnosis of sepsis," Ann. Biomed. Eng. 32, 233-244.

Carvajal, R., Wessel, N., Vallverdu, M., Caminal, P. \& Voss, A. [2005] "Correlation dimension analysis of heart rate variability in patients with dilated cardiomyopathy," Comput. Meth. Progr. Biomed. 78, 133-140.

Cerutti, S., Carrault, G., Cluitmans, P. J., Kinie, A., Lipping, T., Nikolaidis, N., Pitas, I. \& Signorini, M. G. [1996] "Non-linear algorithms for processing biological signals," Comput. Meth. Progr. Biomed. 51, $51-73$.

Chen, R. \& Tsay, R. [1993] "Nonlinear additive arx models," J. Am. Stat. Assoc. 88, 955-967.

Chialvo, D. R. [2002] "Unhealthy surprises," Nature 419, p. 263.

Cohen, I. M., O'Connor, D. T., Preston, R. A. \& Stone, R. A. [1981] "Long-term clonidine effects on autonomic function in essential hypertensive man," Eur. J. Clin. Pharmacol. 19, 25-32.

Costa, M., Goldberger, A. L. \& Peng, C. K. [2002] "Multiscale entropy analysis of complex physiologic time series," Phys. Rev. Lett. 89, 068102.

Costa, M., Goldberger, A. L. \& Peng, C. K. [2005] "Multiscale entropy analysis of biological signals," Phys. Rev. E 71, 021906.

Damiano, R. J. J. [1992] "Implantable cardioverter defibrillators: Current status and future directions," J. Card. Surg. 7, 36-57.

Di Rienzo, M., Bertinieri, G., Mancia, G. \& Pedotti, A. [1985] "A new method for evaluating the baroreflex role by a joint pattern analysis of pulse interval and systolic blood pressure series," Med. Biol. Eng. Comput. 23, 313-314.

Ditto, W. L. \& Showalter, K. [1998] "Introduction: Control and synchronization of chaos," Chaos 7, 509-511. 
Ducher, M., Fauvel, J. P., Gustin, M. P., Cerutti, C., Najem, R., Cuisinaud, G., Laville, M., Pozet, N. \& Paultre, C. Z. [1995] "A new non-invasive statistical method to assess the spontaneous cardiac baroreflex in humans," Clin. Sci. (Lond) 88, 651-655.

Ebeling, W., Molgedey, L., Kurths, J. \& Schwarz, U. [2002] "Entropy, complexity, predictability and data analysis of time series and letter sequences," in The Science of Disasters, eds. Bunde, A., Kropp, J. \& Schellnhuber, H. J. (Springer, Berlin), pp. 2-25.

Eckberg, D. L., Drabinsky, M. \& Braunwald, E. [1971] "Defective cardiac parasympathetic control in patients with heart disease," N. Engl. J. Med. 285, 877-883.

Eckmann, J.-P., Kamphorst, S. O. \& Ruelle, D. [1987] "Recurrence plots of dynamical systems," Europhys. Lett. 4, 973-977.

Eckmann, J. P., Oliffson Kamphorst, S., Ruelle, D. \& Ciliberto, S. [1986] "Lyapunov exponents from time series," Phys. Rev. A 34, 4971-4879.

Einthoven, W. [1902] "Galvanometrische registratie van het menselijk electrocardiogram [galvanometric registration of the human electrogram]," in Herinneringsbundel, ed. Rosenstern, P. S. (Eduard Ijdo, Leiden: The Netherlands), p. 101.

Engbert, R., Scheffczyk, C., Krampe, R., Rosenblum, M., Kurths, J. \& Kliegl, R. [1997] "Tempo-induced transitions in polyrhythmic hand movements," Phys. Rev. E 56, 5823-5833.

Faber, R., Baumert, M., Stepan, H., Wessel, N., Voss, A. \& Walther, T. [2004] "Baroreflex sensitivity, heart rate and blood pressure variability in hypertensive pregnancy disorders," J. Hum. Hypertens. 18, 707712.

Fleisher, L. A., Pincus, S. M. \& Rosenbaum, S. H. [1993] "Approximate entropy of heart rate as a correlate of postoperative ventricular dysfunction," Anesthesiology $\mathbf{7 8}, 683-692$.

Focus Issue: Dynamical disease: Mathematical analysis of human illness [1995] "Focus issue: Dynamical disease: Mathematical analysis of human illness," Chaos 5, 1-344.

Focus Issue: Mapping and control of complex cardiac arrhythmias [2002] "Focus issue: Mapping and control of complex cardiac arrhythmias," Chaos 12, 732-981.

Gao, J. B. [1999] "Recurrence time statistics for chaotic systems and their applications," Phys. Rev. Lett. 83, 3178-3181.

Gao, J. B. \& Cai, H. Q. [2000] "On the structures and quantification of recurrence plots," Phys. Lett. A 270, p. 7587 .

Garfinkel, A., Spano, M. L., Ditto, W. L. \& Weiss, J. N. [1992] "Controlling cardiac chaos," Science 257, 1230-1235.

Gebelein, H. [1941] "Das statistische problem der korrelation als variations- und eigenwertproblem und sein zusammenhang mit der ausgleichsrechnung," Z. Angew. Math. Mech. 21, 364-379.

Glass, L. \& Mackey, M. C. [1988] "Noise and chaos," in From Clocks to Chaos (Princeton University Press, Princeton), p. 1241.

Goldberger, A. L., Findley, L. J., Blackburn, M. R. \& Mandell, A. J. [1984] "Nonlinear dynamics in heart failure: Implications of long-wavelength cardiopulmonary oscillations," Am. Heart J. 197, 612-615.

Goldberger, A. L., Rigney, D. R., Mietus, J., Antman, E. M. \& Greenwald, S. [1988] "Nonlinear dynamics in sudden cardiac death syndrome: Heart rate oscillations and bifurcations," Experientia 44, 983-987.

Goldberger, A. L., Amaral, L. A., Glass, L., Hausdorff, J. M., Ivanov, P. C., Mark, R. G., Mietus, J. E., Moody, G. B., Peng, C. K. \& Stanley, H. E. [2000] "Physiobank, physiotoolkit, and physionet: Components of a new research resource for complex physiologic signals," Circulation 101, E215-E220.

Grassberger, P. \& Procaccia, I. [1983] "Characterization of strange attractors," Phys. Rev. Lett. 50, 346-349.

Gray, R. A., Pertsov, A. M. \& Jalife, J. [1998] "Spatial and temporal organization during cardiac fibrillation," Nature 392, 75-78.

Haaksma, J., Dijk, W. A., Brouwer, J., van den Berg, M., Crijns, H. J. G. M. \& Lie, K. I. [1995] "Effects of automatic ectopy exclusion on the analysis of heart rate variability using a percentile exclusion rule," Comput. Cardiol. 22, 197-200.

Hartikainen, J. E., Malik, M., Staunton, A., Poloniecki, J. \& Camm, A. J. [1996] "Distinction between arrhythmic and nonarrhythmic death after acute myocardial infarction based on heart rate variability, signal-averaged electrocardiogram, ventricular arrhythmias and left ventricular ejection fraction," J. Am. Coll. Cardiol. 28, 296-304.

Hastie, T. \& Tibshirani, R. [1990] Generalized Additive Models (Chapman and Hall, London, NY).

Hering, H. [1909] "Experimentelle studie an saugetieren uber das elektrokardiogramm," Zschr. Exper. Path Therapie. 7, 363-378.

Hirschfeld, H. O. [1935] "A connection between correlation and contingency," Proc. Camb. Phil. Soc. 31, 520-524.

Hogue, Jr, C. W., Domitrovich, P. P., Stein, P. K., Despotis, G. D., Re, L., Schuessler, R. B., Kleiger, R. E. \& Rottman, J. N. [1998] "Rr interval dynamics before atrial fibrillation in patients after coronary artery bypass graft surgery," Circulation 98, 429-434.

Ho, K. K., Moody, G. B., Peng, C. K., Mietus, J. E., Larson, M. G., Levy, D. \& Goldberger, A. L. [1997] "Predicting survival in heart failure case and control subjects by use of fully automated methods for deriving nonlinear and conventional indices of heart rate dynamics," Circulation 96, 842-848. 
Huikuri, H. V., Seppanen, T., Koistinen, M. J., Airaksinen, J., Ikaheimo, M. J., Castellanos, A. \& Myerburg, R. J. [1996] "Abnormalities in beat-tobeat dynamics of heart rate before the spontaneous onset of life-threatening ventricular tachyarrhythmias in patients with prior myocardial infarction," Circulation 93, 1836-1844.

Huikuri, H. V., Poutiainen, A. M., Makikallio, T. H., Koistinen, M. J., Airaksinen, K. E., Mitrani, R. D., Myerburg, R. J. \& Castellanos, A. [1999] "Dynamic behavior and autonomic regulation of ectopic atrial pacemakers," Circulation 100, 1416-1422.

Ivanov, P. C., Amaral, L. A., Goldberger, A. L., Havlin, S., Rosenblum, M. G., Struzik, Z. R. \& Stanley, H. E. [1999] "Multifractality in human heartbeat dynamics," Nature 399, 461-465.

Ivanov, P. C., Amaral, L. A., Goldberger, A. L., Havlin, S., Rosenblum, M. G. \& Struzik, Z. R. [2001] "From 1/f noise to multifractal cascades in heartbeat dynamics," Chaos 11, 641-652.

Javorka, M., Zila, I., Balharek, T. \& Javorka, K. [2002] "Heart rate recovery after exercise: relations to heart rate variability and complexity," Braz. J. Med. Biol. Res. 35, 991-1000.

Kantz, H. \& Schreiber, T. [1997] Nonlinear Time Series Analysis (University Press, Cambridge).

Kantz, H., Kurths, J. \& Mayer-Kress, G. E. [1998] Nonlinear Analysis of Physiological Data (Springer, Berlin).

Karemaker, J. M. \& Lie, K. I. [2000] "Heart rate variability: A telltale of health or disease," Eur. Heart J. 21, 435-437.

Kim, J. H., Yi, S. H., Yoo, C. S., Yang, S. A., Yoon, S. C., Lee, K. Y., Ahn, Y. M., Kang, U. G. \& Kim, Y. S. [2004] "Heart rate dynamics and their relationship to psychotic symptom severity in clozapine-treated schizophrenic subjects," Prog. Neuropsychopharmacol. Biol. Psychiatry 28, 371-378.

Kleiger, R. E., Miller, J. P., Bigger, J. \& Moss, A. [1987] "Decreased heart rate variability and its association with increased mortality after acute myocardial infarction," Am. J. Cardiol. 59, 256-262.

Klingenheben, T. \& Hohnloser, S. H. [2003] "Usefulness of risk stratification for future cardiac events in infarct survivors with severely depressed versus near-normal left ventricular function: Results from a prospective long-term follow-up study," Ann. Noninvasive Electrocardiol. 8, 68-74.

Kobayashi, M. \& Musha, T. [1982] "1/f fluctuation of heartbeat period," IEEE Tran. Biomed. Eng. 29, 456-457.

Koebbe, M. \& Mayer-Kress, G. [1992] "Use of the recurrence plots in the analysis of time-series data," in Nonlinear Modeling and Forecasting, eds. Casdagli, M. \& Eubank, S. (Addison Wesley, Redwood City), pp. $361-378$.
Koepchen, H. P. [1959] "Die nervale und hormonale regulation des blutkreislaufs," in Verhandlungen der Deutschen Gesellschaft fuer Herzkreislaufforschung, ed. Thauer, R. (Dr. Dietrich Steinkopf Verlag, Darmstadt), pp. 54-55.

Kopitzki, K., Warnke, P. C. \& Timmer, J. [1998] "Quantitative analysis by renormalized entropy of invasive electroencephalograph recordings in focal epilepsy," Phys. Rev. E 58, 4859-4864.

Kudenchuk, P. J., Maynard, C., Cobb, L. A., Wirkus, M., Martin, J. S., Kennedy, J. W. \& Weaver, W. D. [1998] "Utility of the prehospital electrocardiogram in diagnosing acute coronary syndromes: The myocardial infarction triage and intervention (miti) project," J. Am. Coll. Cardiol. 32, 17-27.

Kupper, N., Willemsen, G., van den Berg, M., de Boer, D., Posthuma, D., Boomsma, D. \& de Geus, E. [2004] "Heritability of ambulatory heart rate variability," Circulation 110, 2792-2796.

Kurths, J. \& Herzel, H. [1987] "An attractor in a solar time series," Physica D 25, 165-172.

Kurths, J., Voss, A., Witt, A., Saparin, P., Kleiner, H. J. \& Wessel, N. [1995] "Quantitative analysis of heart rate variability," Chaos 5, 88-94.

Lakatta, E. G. \& Levy, D. [2003] "Arterial and cardiac aging: Major shareholders in cardiovascular disease enterprises: Part II: The aging heart in health: links to heart disease," Circulation 107, 346-354.

La Rovere, M. T. \& Schwartz, P. J. [1997] "Baroreflex sensitivity as a cardiac and arrhythmia mortality risk stratifier," Pacing Clin. Electrophysiol. 20, 26022613.

La Rovere, M. T., Bigger Jr, J. T., Marcus, F. I., Mortara, A. \& Schwartz, P. J. [1998] "Baroreflex sensitivity and heart-rate variability in prediction of total cardiac mortality after myocardial infarction. Atrami (autonomic tone and reflexes after myocardial infarction) investigators," Lancet 351, 478-484.

La Rovere, M. T., Pinna, G. D., Hohnloser, S. H., Marcus, F. I., Mortara, A., Nohora, R., Bigger, T., Camm, A. J. \& Schwartz, P. J. [2001] "Baroreflex sensitivity and heart rate variability in the identification of patients at risk for life-threatening arrhythmias," Circulation 103, 2072-2077.

La Rovere, M. T., Pinna, G. D., Maestri, R., Mortara, A., Capomolla, S., Febo, O., Ferrari, R., Franchini, M., Gnemmi, M., Opasich, C., Riccardi, P., Traversi, E. \& Corbelli, F. [2003] "Short-term heart variability predicts sudden cardiac death in chronic heart failure patients," Circulation 107, 565-570.

Lee, M. S., Rim, Y. H., Jeong, D. M., Kim, M. K., Joo, M. C. \& Shin, S. H. [2005] "Nonlinear analysis of heart rate variability during qi therapy (external qigong)," Am. J. Chin. Med. 33, 579-588.

Lehtinen, R., Sievanen, H., Viik, J., Turjanmaa, V., Niemela, K. \& Malmivou, J. [1996] "Accurate 
detection of coronary artery disease by integrated analysis of the st-segment depression/heart rate patterns during exercise and recovery phases of the exercise electrocardiography test," Am. J. Cardiol. 7, 1002-1006.

Lewis, T. [1910] "Notes upon alternation of the heart," QJ. Med. 4, 141-144.

Lippman, N., Stein, K. M. \& Lerman, B. B. [1994] "Comparison of methods for removal of ectopy in measurement of heart rate variability," Am. J. Physiol. 267, H411-H418.

Li, W. A bibliography on $1 / f$ noise http://www.nslijgenetics.org/wli/1fnoise/.

Lombardi, F., Pagani, M., Malliani, A. \& Cerutti, S. [1996a] "Heart rate variability and its sympathovagal modulation," Cardiovas. Res. 32, 208-216.

Lombardi, F., Sandrone, G., Mortara, A., Torzillo, D., La Rovere, M. T., Signorini, M. G., Cerutti, S. \& Malliani, A. [1996b] "Linear and nonlinear dynamics of heart rate variability after acute myocardial infarction with normal and reduced left ventricular ejection fraction," Am. J. Cardiol. 77, 1283-1288.

Lorenz, E. N. [1965] "A study of the predictability of a 28-variable atmospheric model," Tellus 17, 321-333.

Ludwig, C. [1847] "Beitraege zur kenntnis des einflusses der respirationsbewegungen auf den blutlauf im aortensystem," Arch. Anat. Physiol. Leipzig 13, 242-302.

Makikallio, T. H., Ristimae, T., Airaksinen, K. E., Peng, C. K., Goldberger, A. L. \& Huikuri, H. V. [1998] "Heart rate dynamics in patients with stable angina pectoris and utility of fractal and complexity measures," Am. J. Cardiol. 81, 27-31.

Makikallio, T. H., Huikuri, H. V., Hintze, U., Videbaek, J., Mitrani, R. D., Castellanos, A., Myerburg, R. J. \& Moller, M. [2001a] "Fractal analysis and timeand frequency-domain measures of heart rate variability as predictors of mortality in patients with heart failure," Am. J. Cardiol. 87, 178-182.

Makikallio, T. H., Huikuri, H. V., Makikallio, A., Sourander, L. B., Mitrani, R. D., Castellanos, A. \& Myerburg, R. J. [2001b] "Prediction of sudden cardiac death by fractal analysis of heart rate variability in elderly subjects," J. Am. Coll. Cardiol. 37, 13951402 .

Malberg, H., Wessel, N., Schirdewan, A., Osterziel, K. J. \& Voss, A. [1999] "Duale sequenzmethode zur analyse der spontanen baroreflexsensitivitt bei patienten mit dilatativer kardiomyopathie," Z. Kardiol. 88, 331-337.

Malberg, H., Wessel, N., Hasart, A., Osterziel, K. J. \& Voss, A. [2002] "Advanced analysis of the spontaneous baroreflex sensitivity, blood pressure and heart rate variability in patients with dilated cardiomyopathy," Clin. Sci. 102, 465-473.
Malberg, H., Bauernschmitt, R., Meyerfeldt, U., Schirdewan, A. \& Wessel, N. [2003] "Kurzzeit-analyse der herzfrequenzturbulenz versus variabilitätsparameter und barorezeptorsensitivität bei patienten mit dilatativer kardiomyopathie," Z. Kardiol. 92, 547-557.

Malberg, H., Bauernschmitt, R., Meyerfeldt, U., Schirdewan, A. \& Wessel, N. [2004] "Short-term heart rate turbulence analysis versus variability and baroreceptor sensitivity in patients with dilated cardiomyopathy," Indian Pacing Electrophysiol. J. 4, 162-175.

Malberg, H. \& Wessel, N. [2006] "Sampling frequency a wrongly neglected parameter," Somnologie 10, 33-35.

Malliani, A., Pagani, M., Lombardi, F. \& Cerutti, S. [1991] "Cardiovascular neural regulation explored in the frequency domain," Circulation 84, 482492.

Mancia, G., Grassi, G., Bertinieri, G., Ferrari, A. \& Zanchetti, A. [1984] "Arterial baroreceptor control of blood pressure in man," J. Auton. Nerv. Syst. 11, $115-124$.

Mancia, G., Grassi, G., Ferrari, A. \& Zanchetti, A. [1985] "Reflex cardiovascular regulation in human," J. Cardiovasc. Pharmacol. 7, 152-159.

Mandelbrot, B. B. [1983] The Fractal Geometry of Nature (French edition published 1975.) (W. H. Freeman, New York).

Man in't Veld, A. J., van Montfrans, G. A., Langewouters, G. J., Lie, K. I. \& Mancia, G. [1995] "In of the two satellite symposia to the seventh meeting of the European society of hypertension," Measurement of Heart Rate and Blood Pressure Variability in Man: Methods, Mechanisms and Clinical Applications of Continuous Finger Blood Pressure Measurement (Van Zuiden Communications B.V.).

Marwan, N. [1999] "Untersuchung der klimavariabilitt in nw argentinien mit hilfe der quantitativen analyse von recurrence plots," Masters Thesis, Dresden University of Technology.

Marwan, N., Wessel, N., Meyerfeldt, U., Schirdewan, A. \& Kurths, J. [2002] "Recurrence-plot-based measures of complexity and their application to heart-ratevariability data," Phys. Rev. E 66, 026702.

McCall, R. B. \& Humphrey, S. J. [1983] "Interactions between baroreceptor and sympathetic pathways," Am. J. Physiol. 244, R455-R465.

Meyerfeldt, U., Wessel, N., Schutt, H., Selbig, D., Schumann, A., Voss, A., Kurths, J., Ziehmann, C., Dietz, R. \& Schirdewan, A. [2002] "Heart rate variability before the onset of ventricular tachycardia: Differences between slow and fast arrhythmias," Int. J. Cardiol. 84, 141-151.

Milhorn, H. A. T. [1966] The Application of Control Theory to Physiological Systems (Saunders, Philadelphia London). 
Mortara, A., La Rovere, M. T., Pinna, G. D., Prpa, A., Maestri, R., Febo, O., Pozzoli, M., Opasich, C. \& Tavazzi, L. [1997] "Arterial baroreflex modulation of heart rate in chronic heart failure: Clinical and hemodynamic correlates and prognostic implications," Circulation 96, 3450-3458.

Nese, J. M. [1989] "Quantifying local predictibility in phase space," Physica D 35, 237-250.

Nollo, G., Faes, L., Porta, A., Antolini, R. \& Ravelli, F. [2005] "Exploring directionality in spontaneous heart period and systolic pressure variability interactions in humans: implications in the evaluation of baroreflex gain," Am. J. Physiol. Heart Circ. Physiol. 288, H1777-H1785.

Nollo, G., Porta, A., Faes, L., Del Greco, M., Disertori, M. \& Ravelli, F. [2001] "Causal linear parametric model for baroreflex gain assessment in patients with recent myocardial infarction," Am. J. Physiol. Heart. Circ. Physiol. 280, H1830-H1839.

Osaka, M., Saitoh, H., Atarashi, H. \& Hayakawa, H. [1993] "Correlation dimension of heart rate variability: A new index of human autonomic function," Front Med. Biol. Eng. 5, 289-300.

Oseledec, V. I. [1968] "A multiplicative ergodic theorem, ljapunov characteristic numbers for dynamical systems," Trans. Moscow Math. Soc. 19, 197231.

Osterziel, K. J., Hanlein, D., Willenbrock, R., Eichhorn, C., Luft, F. \& Dietz, R. [1995] "Baroreflex sensitivity and cardiovascular mortality in patients with mild to moderate heart failure," Br. Heart J. 73, 517522.

Ott, E. [1993] Chaos in Dynamical Systems (Cambridge, University Press).

Pagani, M., Somers, V., Furlan, R., Dell'Orto, S., Conway, J., Baselli, G., Cerutti, S., Sleight, P. \& Malliani, A. [1988] "Changes in autonomic regulation induced by physical training in mild hypertension," Hypertension 12, 600-610.

Parati, G., Di Rienzo, M., Bertinieri, G., Pomidossi, G., Casadei, R., Groppelli, A., Pedotti, A., Zanchetti, A. \& Mancia, G. [1988] "Evaluation of the baroreceptorheart rate reflex by 24-hour intra-arterial blood pressure monitoring in humans," Hypertension 12, 214-222.

Patton, D. J., Triedman, J. K., Perrott, M. H., Vidian, A. A. \& Saul, J. P. [1996] "Baroreflex gain: Characterization using autoregressive moving average analysis," Am. J. Physiol. Heart Circ. Physiol. 270, H1240H1249.

Penaz, J. [1973] "Photoelectric measurement of blood pressure volume and flow in the finger," in Digest of the 10th Int. Conf. Medical and Biological Engineering, International Federation for Medical and Biological Engineering, Dresden, FRG, pp. 104.
Peng, C. K., Buldyrev, S. V., Havlin, S., Simons, M., Stanley, H. E. \& Goldberger, A. L. [1994] "Mosaic organization of DNA nucleotides," Phys. Rev. E 49, $1685-1689$.

Peng, C. K., Havlin, S., Stanley, H. E. \& Goldberger, A. L. [1995] "Quantification of scaling exponents and crossover phenomena in nonstationary heartbeat time series," Chaos 5, 82-87.

Perkiomaki, J. S., Couderc, J. P., Daubert, J. P. \& Zareba, W. [2003] "Temporal complexity of repolarization and mortality in patients with implantable cardioverter defibrillators," Pacing Clin. Electrophysiol. 26, 1931-1936.

Pikovsky, A., Rosenblum, M. \& Kurths, J. [2000] "Phase synchronization in regular and chaotic systems," Int. J. Bifurcation and Chaos 10, 2291-2305.

Pilgram, B. \& Kaplan, D. T. [1999] "Nonstationarity and $1 / f$ noise characteristics in heart rate," Am. J. Physiol. 276, R1-R19.

Pincus, S. M. [1991] "Approximate entropy as a measure of system complexity," Proc. Natl. Acad. Sci. USA 88, 2297-2301.

Pincus, S. M. [2000] "Orderliness of hormone release," Novartis Found Symp. 227, 82-96.

Poon, C. S. \& Merrill, C. K. [1997] "Decrease of cardiac chaos in congestive heart failure," Nature 389, 492-495.

Porta, A., Baselli, G., Rimoldi, O., Malliani, A. \& Pagani, M. [2000] "Assessing baroreflex gain from spontaneous variability in conscious dogs: Role of causality and respiration," Am. J. Physiol. Heart Circ. Physiol. 279, H2558-H2567.

Raab, C. \& Kurths, J. [2001] "Estimation of large-scale dimension densities," Phys. Rev. E 64.

Raab, C., Wessel, N., Schirdewan, A. \& Kurths, J. [2005] "Large-scale dimension densities for heart rate variability analysis," Comput. Cardiol. 32, 985-988.

Raab, C., Wessel, N., Schirdewan, A. \& Kurths, J. [2006] "Large-scale dimension densities for heart rate variability analysis," Phys. Rev. E 73, 041907.

Ramanand, P., Nampoori, V. P. \& Sreenivasan, R. [2004] "Complexity quantification of dense array eeg using sample entropy analysis," J. Integr. Neurosci. 3, 343358.

Renyi, A. [1970] Probability Theory (Akadémiai Kiadó Budapest).

Richman, J. S. \& Moorman, J. R. [2000] "Physiological time-series analysis using approximate entropy and sample entropy," Am. J. Physiol. Heart Circ. Physiol. 278, H2039-H2049.

Robbe, H. W., Mulder, L. J., Ruddel, H., Langewitz, W. A., Veldman, J. B. \& Mulder, G. [1987] "Assessment of baroreceptor reflex sensitivity by means of spectral analysis," Hypertension 10, 538-543.

Rompelman, O. \& Ten Voorde, B. J. [1995] "Modelling and analysis of rapid fluctuations in heart rate and 
blood pressure: A historical perspective," in Measurement of Heart Rate and Blood Pressure Variability in Man: Methods, Mechanisms and Clinical Applications of Continuous Finger Blood Pressure Measurement, eds. Man in't Veld, A. J., van Montfrans, G. A., Langewouters, G. J., Lie, K. I. \& Mancia, G. (Van Zuiden Communications B.V.).

Rosenbaum, D. S., Albrecht, P. \& Cohen, R. J. [1996] "Predicting sudden cardiac death from $t$ wave alternans of the surface electrocardiogram: Promise and pitfalls," J. Cardiovasc. Electrophysiol. 7, 1095-1111.

Saparin, P., Witt, A., Kurths, J. \& Anishchenko, V. [1994] "The renormalized entropy - An appropriate complexity measure," Chaos Solit. Fract. 4, 19071916.

Schafer, C., Rosenblum, M., Kurths, J. \& Abel, H. [1998] "Heartbeat synchronized with ventilation," Nature 392, 239-240.

Schirdewan, A., Meyerfeldt, U., Wessel, N., Bondke, H. J., Schreiber, P., Sadowski, R., Kamke, W. \& Wiedemann, M. [2004] "Heart rate dynamics before the onset of ventricular tachyarrhythmias: Results of the cardioverter defibrillator registry marita," J. Am. Coll. Cardiol. 43, 125A-126A.

Schmidt, G., Malik, M., Barthel, P., Schneider, R., Ulm, K., Rolnitzky, L., Camm, A. J., Bigger, J. T. J. \& Schomig, A. [1999a] "Heart-rate turbulence after ventricular premature beats as a predictor of mortality after acute myocardial infarction," Lancet 353, 13901396.

Schmidt, G., Malik, M., Barthel, P., Schneider, R., Ulm, K., Rolnitzky, L., Camm, A. J., Bigger Jr, J. T., \& Schömig, A. [1999b] "Heart rate turbulence after ventricular premature beats as a predictor of mortality after acute myocardial infarction," Lancet $\mathbf{3 5 3}$, 1390-1396.

Schwarz, U., Benz, A., Kurths, J. \& Witt, A. [1993] "Analysis of solar spike events by means of symbolic dynamics methods," Astron. Astrophys. 277, 215224.

Smith, L. A. [1994] "Local optimal prediction: Exploiting strangeness and the variation of sensitiy to initial condition," Phil. Trans. R. Soc. Lond. A 348, 371381.

Special Issue: Virtual Tissue Engineering of the Heart [2003] "Special issue: Virtual tissue engineering of the heart," Int. J. Bifurcation and Chaos 13, 3531-3804.

Stanley, H. E., Amaral, L. A., Goldberger, A. L., Havlin, S., Ivanov, P. \& Peng, C. K. [1999] "Statistical physics and physiology: Monofractal and multifractal approaches," Physica A 270, 309-324.

Steinbigler, P., Haberl, R., Jilge, G. \& G., S. [1998] "Single-beat analysis of ventricular late potentials in the surface electrocardiogram using the spectrotemporal pattern recognition algorithm in patients with coronary artery disease," Eur. Heart J. 19, 435-446.
Sugiura, T., Mizushina, S., Kimura, M., Fukui, Y. \& Harada, Y. [1991] "A fuzzy approach to the rate control in an artificial cardiac pacemaker regulated by respiratory rate and temperature: A preliminary report," J. Med. Eng. Technol. 15, 107-110.

Suurkula, M., Arvidsson, A., Fagerberg, B., Bjuro, T. \& Wikstrand, J. [2001] "A new method to quantify postexercise st-deviation: s study in man at high and low risk for coronary heart disease," Clin. Physiol. 21, 541-555.

Szabo, B. M., van Veldhuisen, D. J., van der Veer, N., Brouwer, J., De Graeff, P. A. \& Crijns, H. J. [1997] "Prognostic value of heart rate variability in chronic congestive heart failure secondary to idiopathic or ischemic dilated cardiomyopathy," Am. J. Cardiol. 79, 978-980.

Takahashi, N., Nakagawa, M., Saikawa, T., Ooie, T., Akimitsu, T., Kaneda, K., Hara, M., Iwao, T., Yonemochi, H., Ito, M. \& Sakata, T. [1999] "Noninvasive assessment of the cardiac baroreflex: Response to downward tilting and comparison with the phenylephrine method," J. Am. Coll. Cardiol. 34, 211215.

Takens, F. [1981] "Detecting strange attractors in turbulence," in Dynamical Systems and Turbulence, Warwick (1980), Lecture Notes in Mathematics, Vol. 898, eds. Rand, E. \& Young, L. S. (Springer Verlag, Berlin), pp. 366-381.

Task Force Heart Rate Variability [1996] "Task force of the European society of cardiology and the north American society of pacing and electrophysiology," Circulation 93, 1043-1065.

Tateno, K. \& Glass, L. [2001] "A method for detection of atrial fibrillation using rr intervals," Med. Biol. Eng. Comput. 39, 664-671.

Trulla, L. L., Giuliani, A., Zbilut, J. P. \& Webber Jr, C. L. [1996] "Recurrence quantification analysis of the logistic equation with transients," Phys. Lett. A 223, 255-260.

Tsuji, H., Larson, M. G., Venditti, F. J., Manders, E. S., Evans, J. C., Feldman, C. L. \& Levy, D. [1996] "Impact on reduced heart rate variability on risk for cardiac events," Circulation 94, 2850-2855.

Vanoli, E. \& Adamson, P. B. [1994] "Baroreflex sensitivity: Methods, mechanisms, and prognostic value," Pacing Clin. Electrophysiol. 17, 434-445.

Verdecchia, P., Clement, D., Fagard, R., Palatini, P. \& Parati, G. [1999] "Blood pressure monitoring. Task force III: Target-organ damage, morbidity and mortality," Blood Press Monit. 4, 303-317.

Vikman, S., Makikallio, T. H., Yli-Mayry, S., Pikkujamsa, S., Koivisto, A. M., Reinikainen, P., Airaksinen, K. E. \& Huikuri, H. V. [1999] "Altered complexity and correlation properties of rr interval dynamics before the spontaneous onset of paroxysmal atrial fibrillation," Circulation 100, 2079-2084. 
Vikman, S., Lindgren, K., Makikallio, T. H., YliMayry, S., Airaksinen, K. E. \& Huikuri, H. V. [2005] "Heart rate turbulence after atrial premature beats before spontaneous onset of atrial fibrillation," J. Am. Coll. Cardiol. 45, 278-284.

Voss, A., Busjahn, A., Wessel, N., Schurath, R., Faulhaber, D., Luft, F. C. \& Dietz, R. [1996a] "Familial and genetic influences on heart rate variability," $J$. Electrocardiol. 29, 154-160.

Voss, A., Kurths, J., Kleiner, H. J., Witt, A., Wessel, N., Saparin, P., Osterziel, K. J., Schurath, R. \& Dietz, R. [1996b] "The application of methods of non-linear dynamics for the improved and predictive recognition of patients threatened by sudden cardiac death," Cardiovasc. Res. 31, 419-433.

Voss, A., Wessel, N., Sander, A. \& Malberg, H. [1996c] "Requirements on sampling rate in holter systems for analysis of heart rate variability," Clin. Sci. (Colch) 91, 120-121.

Voss, H. \& Kurths, J. [1997] "Reconstruction of nonlinear time delay models from data by the use of optimal transformations," Phys. Lett. A 234, 336-344.

Voss, A., Hnatkova, K., Wessel, N., Kurths, J., Sander, A., Schirdewan, A., Camm, A. J. \& Malik, M. [1998] "Multiparametric analysis of heart rate variability used for risk stratification among survivors of acute myocardial infarction," Pacing Clin. Electrophysiol. 21, 186-192.

Voss, H. U., Kolodner, P., Abel, M. \& Kurths, J. [1999] "Amplitude equations from spatiotemporal binaryfluid convection data," Phys. Rev. Lett. 83, 3422-3425.

Voss, A., Malberg, H., Schumann, A., Wessel, N., Walther, T., Stepan, H. \& Faber, R. [2000] "Baroreflex sensitivity, heart rate, and blood pressure variability in normal pregnancy," Am. J. Hypertens. 13, $1218-1225$.

Voss, H., Timmer, J. \& Kurths, J. [2004] "Nonlinear dynamic system identification from uncertain and indirect measurements," Int. J. Bifurcation and Chaos 14, 1905-1933.

Wackerbauer, R., Witt, A., Atmanspacher, H., Kurths, J. \& Scheingraber, H. [1994] "A comparative classification of complexity measures," Chaos Solit. Fract. 4, 133-173.

Walther, T., Wessel, N., Baumert, M., Stepan, H., Voss, A. \& Faber, R. [2005] "Longitudinal analysis of heart rate variability in chronic hypertensive pregnancy," Hypertens. Res. 28, 113-118.

Walther, T., Wessel, N., Malberg, H., Voss, A., Stepan, H. \& Faber, R. [2006] "Effective prediction of preeclampsia: Concurrent measurement of uterine perfusion and analysis of heart rate and blood pressure variability," J. Hypertens. 24, 747-750.

Webber, C. L. \& Zbilut, J. P. [1994] "Dynamical assessment of physiological systems and states using recurrence plot strategies," J. Appl. Physiol. 76, 965-973.
Weisfeldt, M. L., Edward, G. L. \& Gerstenblith, G. [1992] "Aging and the heart," in Heart Disease: A Textbook of Cardiovascular Medicine, ed. Braunwald, E. (WB Saunders, Philadelphia), pp. 16561669.

Wernicke, D., Wessel, N., Malberg, H., Plehm, R., Bauernschmitt, R. \& Thierfelder, L. [2007] $\alpha$ tropomyosin mutations asp175asn and glu180gly differently affect cardiac autonomic regulation in transgenic rats," Biomed. Tech. (Berl) 52, 50-55.

Wessel, N., Voss, A., Kurths, J., Saparin, P., Witt, A., Kleiner, H. J. \& Dietz, R. [1994] "Renormalised entropy: A new method of non-linear dynamics for the analysis of heart rate variability," Comput. Cardiol. 21, 137-140.

Wessel, N., Voss, A., Kurths, J., Schirdewan, A., Hnatkova, K. \& Malik, M. [2000a] "Evaluation of renormalised entropy for risk stratification using heart rate variability data," Med. Biol. Eng. Comput. 38, 680-685.

Wessel, N., Voss, A., Malberg, H., Ziehmann, C., Voss, H., Schirdewan, A., Meyerfeldt, U. \& Kurths, J. [2000b] "Nonlinear analysis of complex phenomena in cardiological data," Herzschr. Elektrophys. 11, 159-173.

Wessel, N., Ziehmann, C., Kurths, J., Meyerfeldt, U., Schirdewan, A. \& Voss, A. [2000c] "Short-term forecasting of life-threatening cardiac arrhythmias based on symbolic dynamics and finite-time growth rates," Phys. Rev. E 61, 733-739.

Wessel, N., Schirdewan, A. \& Kurths, J. [2003] "Intermittently decreased beat-to-beat variability in congestive heart failure," Phys. Rev. Lett. 91, 119801.

Wessel, N., Aßmus, J., Weidermann, F., Konvicka, J., Nestmann, S., Neugebauer, R., Schwarz, U. \& Kurths, J. [2004a] "Modeling thermal displacements in modular tool systems," Int. J. Bifurcation and Chaos 14, 2125-2132.

Wessel, N., Konvicka, J., Weidermann, F., Nestmann, S., Neugebauer, R., Schwarz, U., Wessel, A. \& Kurths, J. [2004b] "Predicting thermal displacements in modular tool systems," Chaos 14, 23-29.

Wessel, N., Malberg, H. \& Walther, T. [2004c] "Heart rate turbulence higher predictive value than other risk stratifiers?" Circulation 109, e150-e151.

Wessel, N., Malberg, H., Bauernschmitt, R., Schirdewan, A. \& Kurths, J. [2006] "Nonlinear autoregressive model-based analysis of short-term heart rate variability," Med. Biol. Eng. Comput. 44, 321330.

Westerhof, B. E., Gisolf, J., Stok, W. J., Wesseling, K. H. \& Karemaker, J. M. [2004] "Time-domain cross-correlation baroreflex sensitivity: Performance on the eurobavar data set," J. Hypertens. 22, 13711380.

Wikswo, J. P. J., Lin, S. F. \& Abbasm, R. A. [1995] "Virtual electrodes in cardiac tissue: A common 
mechanism for anodal and cathodal stimulation," Biophys. J. 69, 2195-2210.

Witkowski, F. X., Leon, L. J., Penkoske, P. A., Giles, W. R., Spano, M. L., Ditto, W. L. \& Winfree, A. T. [1998] "Spatiotemporal evolution of ventricular fibrillation," Nature 392, 78-82.

Wolf, A., Swift, J. B., Swinney, H. L. \& Vastano, J. A. [1985] "Determining lyapunov exponents from a time series," Physica D 16, 285-317.

Wolf, M. W., Varigos, G. A., Hunt, D. \& Sloman, J. G. [1978] "Sinus arrhythmia in acute myocardial infarction," Med. J. Austral. 2, 52.

Woo, M. A., Stevenson, W. G. \& Moser, D. K. Middlekauff, H. R. [1994] "Complex heart rate variability and serum norepinephrine levels in patients with advanced heart failure," J. Am. Coll. Cardiol. 23, 56-59.

Yamamoto, Y. \& Hughson, R. L. [1994a] "On the fractal nature of heart rate variability in humans: Effects of data length and beta-adrenergic blockade," Am. J. Physiol. 266, R40-R49.

Yamamoto, Y. \& Hughson, R. L. [1994b] "On the fractal nature of heart rate variability in humans: Effects of data length and beta-adrenergic blockade," Am. J. Physiol. 266, R40-R49.
Yang, A. C., Hseu, S. S., Yien, H. W., Goldberger, A. L. \& Peng, C. K. [2003] "Linguistic analysis of the human heartbeat using frequency and rank order statistics," Phys. Rev. Lett. 90, 108103.

Yoden, S. \& Nomura, M. [1993] "Finite-time lyapunov stability analysis and its application to atmospheric predictability," J. Atmosph. Sci. 50, 1531-1543.

Zareba, W., Maison-Blanche, P. \& Locati, E. H. [2001] Noninvasive Electrocardiology in Clinical Practice (Futura Publishing Co, NY).

Zbilut, J. P. \& Webber Jr, C. L. [1992] "Embeddings and delays as derived from quantification of recurrence plots," Phys. Lett. A 171, 199-203.

Zebrowski, J. J., Poplawska, W., Baranowski, R. \& Buchner, T. [1995] "Local time properties of 24-hour holter recordings of rr intervals: A nonlinear quantitative method trough pattern entropy of 3-d return maps," Comput. Cardiol. 22, 377-380.

Ziehmann, C., Smith, L. A. \& Kurths, J. [1999] "The bootstrap and lyapunov exponents in deterministic chaos," Physica D 126, 49-59.

Zipes, D. P. \& Wellens, H. J. [1998] "Sudden cardiac death," Circulation 98, 2334-2351. 